\title{
Tumbling and Technicolor Theory ${ }^{1}$
}

\author{
Noriaki Kitazawa $^{2}$ \\ Department of Physics, Nagoya University \\ Nagoya, 464-01, Japan \\ January, 1994 \\ DPNU-94-03
}

${ }^{1}$ Doctoral thesis

${ }^{2}$ JSPS fellow 


\begin{abstract}
The extended technicolor theory is a candidate of the physics beyond the standard model. To explain the mass hierarchy of the quarks and leptons, the extended technicolor gauge symmetry must hierarchically break to the technicolor gauge symmetry. Tumbling gauge theory is considered as a candidate of the dynamics of such hierarchical symmetry breaking, since the sequential self-breaking of the gauge symmetry ("tumbling") can be expected in that theory.

It is well known that the extended technicolor theory induces too strong flavorchanging neutral current interactions to be consistent with the experiments. This problem can be solved if the technicolor dynamics is the special one with very large anomalous dimension of the composite operator $\bar{T} T$ composed by the technifermion field $T$. Two types of the models with large anomalous dimension have been proposed. One is the gauge theory with slowly running coupling, another is the gauge theory with strong four fermion interaction. It is expected that the large anomalous dimension is realized in the tumbling gauge theory.

In this thesis we systematically estimate the strength of the effective four fermion interactions induced in the tumbling gauge theory by using the effective action within the one-gauge-boson-exchange approximation. It is shown that the couplings of the effective four fermion interactions cannot be large enough to realize sufficiently large anomalous dimension. This result is important for the model building of the extended technicolor theory. The decoupling of the gauge bosons and fermions which get their masses in the process of tumbling is also discussed. It is non-perturbatively shown that their effect on the low energy dynamics is negligibly small.

Before the estimations, we give the explanation of the fundamental techniques to treat the dynamical symmetry breaking. The scenario and the phenomenology of the extended technicolor theory is also reviewed. The restriction on the extended technicolor theory from the precision experiments is discussed. It is emphasized that the non-oblique correction is important as well as the oblique correction, since the top quark is heavy.
\end{abstract}




\section{Chapter 1}

\section{Introduction}

The standard model of the elementary particle physics almost perfectly explains many present experiments. Especially, the agreement with the recent precision experiments in LEP is significant. The unified description of electromagnetic interaction and weak interaction [1] is now established [. These interactions are described as the gauge interaction of the symmetry $S U(2)_{L} \times U(1)_{Y}$ (electroweak symmetry) which contains four massless gauge bosons. This gauge symmetry is spontaneously broken to the electromagnetic gauge symmetry $U(1)_{e m}$, and three gauge bosons (weak gauge bosons) get their mass by the Higgs mechanism[2]. The mass of these gauge bosons explains why the weak interaction is short range. The electroweak symmetry forbids the mass of quarks and leptons. Quarks and leptons cannot be massive till the electroweak symmetry breaking is achieved. Therefore, the mechanism of the mass generation must tightly be related with the mechanism of the electroweak symmetry breaking.

In the standard model, electroweak symmetry is spontaneously broken by the vacuum expectation value of elementary Higgs field. The magnitude of vacuum expectation value decides the masses of weak gauge bosons. The masses of fermions (quarks and leptons) are generated through the Yukawa interactions between the fermion fields and Higgs field. The mass mixing among the quarks with the same electric charge is also generated. This mixing is the origin of Cabibbo-Kobayashi-Maskawa (CKM) mixing angles and CP violation in the weak interaction[3].

This mechanism of electroweak symmetry breaking and fermion mass generation contains many arbitrary parameters. The masses of fermions, CKM mixing angles, and CP violating phase cannot be predicted in the framework of the standard model. This suggests that there are some unknown physics which explain the origin of fermion masses as well as the origin of CKM mixing and $\mathrm{CP}$ violation in the weak interaction. We must make some efforts to find this new physics, though it is a difficult problem.

The approaches to solve this problem may be classified into two categories. One is the solution in the grand unified theories (GUTs) [4. The Yukawa couplings as well as the gauge couplings must be unified at some very high energy scale in this theories. The variety of the Yukawa couplings at the low energy is due to the complex particle contents. The interactions originated by the physics at the Plank scale may also be important 5]. This type of solutions kinematically explain the variety of fermion masses.

\footnotetext{
${ }^{1}$ More rigorously, the final establishment will be done by LEPII experiment.
} 
Another solution dynamically explains the fermion masses. The extended technicolor theory [6] is contained in this category. The theory is based on the technicolor theory [7] in which the electroweak symmetry is dynamically broken by the technifermion condensates due to the strong coupling technicolor interaction. In the technicolor theory the interaction which correspond to the Yukawa interactions are the effective four-fermion interactions between the ordinary fermions and technifermions. In the extended technicolor theory, the effective four-fermion interactions are dynamically generated. The variety of the fermion masses is originated in the dynamics of extended technicolor interaction. The top condensation model[\&] and composite model of quarks and leptons are also contained in this category.

To our regret, both approaches are not completely successful. While the approach with GUTs can describe the fermion masses, CKM mixing angles, and CP violation, the potential of vacuum is very complicated one, and many fine-tuned parameters should be contained in it. We must consider the physics at GUT scale and above to answer the question why the potential is so complicated.

The dynamical approach also is not successful. One of the reason is that the nonperturbative treatment of the strong coupling gauge theory is quite difficult. Since our knowledge of the dynamical spontaneous symmetry breaking is very limited, we must put some postulates to make models. Other reason is that the model tends to become very complicated one to explain the complicated fermion mass spectrum. Many people are disgusted by complicated model with many postulations.

There is no definite reason to select which approach is better, though recent precision experiments at LEP favor the GUT approach (with supersymmetry) but not dynamical approach. It is interesting that the dynamical approach predicts new rich physics (new particles, new interactions) just above the present accessible energy. It is now the time to extensively study this approach, since many intended experiments can be accessible to this new physics.

It is very important to estimate the non-perturbative behavior (dynamics) of the gauge theory for the dynamical approach. In this thesis we treat an interesting gauge theory which can be applied as the dynamics of the extended technicolor theory. It is called tumbling gauge theory [9] in which many dynamical gauge symmetry breakings with different energy scales are expected. Natural existence of such many different mass scales has been expected as the source of many different fermion masses. We formulate this theory by using the effective action in one-gauge-boson-exchange approximation, and estimate the strength (energy scale) of the effective four fermion interactions due to the massive gauge boson exchange. The strength is directly related with the fermion masses.

The strength is also important to solve the flavor-changing neutral current (FCNC) problem. In the naive model, extended technicolor theory generates large FCNC interactions. The extended technicolor gauge bosons and the pseudo-Nambu-Goldstone bosons cause too large $K^{0}-\bar{K}^{0}$ mixing, for example. We may avoid this problem by devising the gauge group and the representation of fermions, but the model should become very complicated one. This problem can simply be solved if the technicolor dynamics is the one with large $\gamma_{m}$, where $\gamma_{m}$ is the anomalous dimension of composite operator $\bar{T} T$ composed by the technifermion field $T[10]$.

Two types of large $\gamma_{m}$ dynamics have been proposed. One is the gauge theory with 
slowly running coupling (so called walking gauge theory) 11] and another is the gauge theory with strong four-fermion interaction [12]. Since many effective four-fermion interactions are induced in tumbling gauge theory, the second type of large $\gamma_{m}$ dynamics is likely be realized. But we show that the effective four-fermion interaction is too weak to realize sufficiently large $\gamma_{m}$. This result is important for the model building of the extended technicolor theory.

We see that the massive gauge bosons can be neglected, when we consider the low energy dynamics. The critical gauge coupling of the fermion pair condensation and the anomalous dimension $\gamma_{m}$ do not change, whether the massive gauge bosons are considered or not. The massive fermions which get their masses in the process of tumbling can also be neglected, when we consider the low energy dynamics. The critical gauge coupling of the pair condensation channel with the massive fermions is too large to be formed the condensate. Although such decoupling of the heavy particles has already been proved based on the the perturbation theory, we non-perturbatively show that in the tumbling gauge theory by using our formalism in the one-gauge-boson exchange approximation.

This thesis is developed as follows. In the next chapter, we review the fundamental properties of realization of symmetry in nature. The fundamentals about spontaneous symmetry breaking and Higgs mechanism is reviewed. In chapter 3, the fundamental properties of dynamical symmetry breaking is described. Various techniques of the nonperturbative treatment of dynamics are reviewed. In chapter 4 and 5, the technicolor models and its phenomenology are reviewed, respectively. The relation with present precision experiments is also considered. In chapter 6, our formalism of tumbling gauge theory is developed. In chapter 7 , the strength of effective four-fermion interaction is estimated, and the possibility of the large anomalous dimension dynamics is discussed. The non-perturbative decoupling is also discussed. Chapter 8 is devoted to the conclusion. 


\section{Chapter 2}

\section{Realization of Symmetry}

The symmetry is an important concept in the elementary particle physics. Many hadrons composed by the quarks have been classified and understood by virtue of the symmetry in QCD. We know that some decay processes of hadrons are forbidden by the conservation law, and the conservation law is directly connected with the symmetry. To find the symmetry in nature has been a very important task to understand the particle spectrum and interactions.

The symmetry is described as the invariance of Lagrangian under the transformation f. For example, the Lagrangian of complex-valued scalar field

$$
\mathcal{L}=\left(\partial_{\mu} \phi\right)^{\dagger}\left(\partial^{\mu} \phi\right)-m^{2} \phi^{\dagger} \phi-\lambda\left(\phi^{\dagger} \phi\right)^{2}
$$

has a global symmetry, since this is invariant under the global transformation

$$
\phi \longrightarrow \phi^{\prime}=e^{i \theta} \phi,
$$

where $\theta$ is the parameter of transformation. We can extend this symmetry to the local one by introducing gauge field $A_{\mu}$. The Lagrangian

$$
\mathcal{L}=\left(D_{\mu} \phi\right)^{\dagger}\left(D^{\mu} \phi\right)-m^{2} \phi^{\dagger} \phi-\lambda\left(\phi^{\dagger} \phi\right)^{2}-\frac{1}{4} F_{\mu \nu} F^{\mu \nu}
$$

is invariant under the local transformation

$$
\begin{aligned}
\phi & \longrightarrow \phi^{\prime}=e^{i \theta(x)} \phi, \\
A_{\mu} & \longrightarrow A_{\mu}^{\prime}=A_{\mu}+\partial_{\mu} \theta(x),
\end{aligned}
$$

where

$$
\begin{aligned}
D^{\mu} \phi & =\partial^{\mu} \phi-i g A^{\mu} \phi, \\
F^{\mu \nu} & =\partial^{\mu} A^{\nu}-\partial^{\nu} A^{\mu},
\end{aligned}
$$

and $g$ is the coupling constant of the gauge interaction.

\footnotetext{
${ }^{1}$ More accurately, we should consider the action instead of the Lagrangian. But here, we consider the Lagrangian for simplicity.
} 
The current and charge of the symmetry are defined as follows. Consider the general fields $\Phi_{i}$ and the (infinitesimal) transformation

$$
\Phi_{i} \longrightarrow \Phi_{i}^{\prime}=\Phi_{i}+\theta^{a}(\delta \Phi)_{i}^{a}
$$

where index $i$ denotes the flavor of the fields and $a$ is the index of the transformation. The current is defined as

$$
J_{\mu}^{a}(x) \equiv-\frac{\partial \mathcal{L}}{\partial\left(\partial_{\mu} \Phi_{i}\right)}(\delta \Phi)_{i}^{a},
$$

and the corresponding charge is defined as

$$
Q^{a} \equiv-\int J_{0}^{a}(x) d^{3} x
$$

by using this current.

We can show that the divergence of the currents are the variation of the Lagrangian by using Eular-Lagrange equation, namely

$$
\partial^{\mu} J_{\mu}^{a}=-(\delta \mathcal{L})^{a}
$$

where the Lagrangian is transformed as

$$
\mathcal{L} \rightarrow \mathcal{L}+(\delta \mathcal{L})^{a}
$$

Therefore, if the Lagrangian is invariant under the transformation $\mathrm{f}$, the divergence of the current vanishes. The current corresponding to the symmetry transformation is called Noether current. The vanishing of the divergence of the current suggests the conservation of corresponding charge, because the charge is independent of time,

$$
\begin{aligned}
\frac{d}{d t} Q^{a} & =-\int \frac{d}{d t} J_{0}^{a} d^{3} x \\
& =\int \nabla \cdot \mathrm{j}^{a} d^{3} x=0 .
\end{aligned}
$$

Here we assume as the boundary condition that the fields and its derivatives vanish at infinity.

The charges generate the transformation when we consider the fields as operators in canonical quantization,

$$
\left[Q^{a}, \Phi_{i}\right]=\frac{1}{i}(\delta \Phi)_{i}^{a}
$$

The charge of the state is defined as the eigenvalue of the charge operator with respect to the corresponding state vector. The transformations are classified by the algebra of the charge operators. If the charge operators, for instance, satisfy the algebra

$$
\left[Q^{a}, Q^{b}\right]=i \epsilon^{a b c} Q^{c}
$$

\footnotetext{
${ }^{2}$ For the invariance of the action, the variation of the Lagrangian do not always have to vanish. The form of the total divergence, $(\delta \mathcal{L})^{a}=\partial^{\mu} X_{\mu}^{a}$, is also acceptable.
} 
the transformation forms the group $S U(2)$, where $a, b$, and $c$ run over 1,2 , and 3 , and $\epsilon^{a b c}$ is the Levi-Civita symbol. Therefore, the symmetry can be classified by the group theory. The charges form the generators of the group.

The symmetry of the system (Lagrangian) can be realized in nature in two ways: Wigner phase or Nambu-Goldstone phase. The generators of the symmetry annihilate the vacuum state in the Wigner phase, but not in Nambu-Goldstone phase. Namely,

$$
\begin{array}{ll}
Q|0\rangle=0 & \text { Wigner phase, } \\
" Q|0\rangle \neq 0 " & \text { Nambu-Goldstone phase }
\end{array}
$$

where the vacuum state is defined by the annihilation operators of the asymptotic fields of the theory. The double quotations in eq.(2.16) means that the formula is just a symbolic one.

Wigner phase is the naive realization of the symmetry. In this phase the charge of the state is conserved in the physical processes. Since the Hamiltonian $\mathcal{H}$ commutes with the charge,

$$
\begin{aligned}
0 & =\langle\alpha|[Q, \mathcal{H}]| \beta\rangle \\
& =\left(Q_{\alpha}-Q_{\beta}\right)\langle\alpha|\mathcal{H}| \beta\rangle,
\end{aligned}
$$

where $|\alpha\rangle$ and $|\beta\rangle$ are the eigenstates of the charge $Q$ with eigenvalues $Q_{\alpha}$ and $Q_{\beta}$, respectively. This means that the transition amplitude do not vanish only if the charges of initial and finial state is equal to each order.

But it is not the case in the Nambu-Goldstone phase. If there exists at least an operator $\Phi$ which satisfies the condition

$$
\begin{aligned}
& {[Q, \Phi]=\delta \Phi \neq 0} \\
& \langle 0|\delta \Phi| 0\rangle \neq 0 .
\end{aligned}
$$

the symmetry is realized as Nambu-Goldstone phase. The generator $Q$ does not annihilate the vacuum state, and then it is not the well-defined one. Since $Q$ commutes with the energy-momentum operator, the state $Q|0\rangle$ must proportional to the vacuum state $|0\rangle$ ? Therefore,

$$
\begin{aligned}
c & =\langle 0|Q| 0\rangle \\
& =-\int\left\langle 0\left|J_{0}(x)\right| 0\right\rangle d^{3} x \\
& =-\int\left\langle 0\left|J_{0}(0)\right| 0\right\rangle d^{3} x=0,
\end{aligned}
$$

where $c$ is a constant. In the last equality, we use the fact that there is no constant Lorentz vector in the theory. If the generator $Q$ is well-defined one, then the constant $c$ must be zero. Namely, if the generator $Q$ does not annihilate the vacuum state, then the generator is not well-defined one. In this phase the symmetry is spontaneously broken,

\footnotetext{
${ }^{3}$ Here, we postulate that the vacuum state is an unique zero energy state. But the result is not changed, if the other independent zero energy states exist.
} 
and the charge of the state as the eigenvalue of the generators cannot be defined. The generator $Q$ is called the broken generator.

The symmetry is realized in the different way from the charge conservation in the Wigner phase. The massless Nambu-Goldstone bosons corresponding to the broken generators exist in particle spectrum. Nambu-Goldstone theorem says that if the conditions

1. Existence of the translational invariance and Lorentz covariance.

2. Existence of the conserved current $j_{\mu}$.

3. Existence of the operator $\Phi$ which satisfies $\langle 0|[Q, \Phi]| 0\rangle=\frac{1}{i}\langle 0|\delta \Phi| 0\rangle \neq 0$ (finite).

are satisfied, the massless Nambu-Goldstone boson exists and it couples to the current.

The proof is the following. Because of the current conservation,

$$
\langle 0|[Q, \Phi(0)]| 0\rangle=\frac{1}{i}\langle 0|\delta \Phi(0)| 0\rangle
$$

is independent of $x_{0}$, where $Q$ is defined as

$$
Q=-\int j_{0}(x) d^{3} x
$$

The left-hand side of eq.(2.20) can be expressed as

$$
\begin{aligned}
\langle 0|[Q, \Phi(0)]| 0\rangle= & -\int d^{3} x \sum_{n}\left\{\left\langle 0\left|j_{0}(x)\right| n\right\rangle\langle n|\Phi(0)| 0\rangle-\langle 0|\Phi(0)| n\rangle\left\langle n\left|j_{0}(x)\right| 0\right\rangle\right\} \\
= & -\int d^{3} x \sum_{n}\left\{\left\langle 0\left|j_{0}(0)\right| n\right\rangle\langle n|\Phi(0)| 0\rangle e^{-i p_{n} x}\right. \\
& \left.-\langle 0|\Phi(0)| n\rangle\left\langle n\left|j_{0}(0)\right| 0\right\rangle e^{i p_{n} x}\right\} \\
= & -\sum_{n}(2 \pi)^{3} \delta^{3}\left(p_{n}\right)\left\{\left\langle 0\left|j_{0}(0)\right| n\right\rangle\langle n|\Phi(0)| 0\rangle e^{-i E_{n} x^{0}}\right. \\
\left.-\langle 0|\Phi(0)| n\rangle\left\langle n\left|j_{0}(0)\right| 0\right\rangle e^{i E_{n} x^{0}}\right\} & -\sum_{n_{1}} \int \frac{d^{3} p_{n_{1}}}{2 E_{n_{1}}} \delta^{3}\left(p_{n_{1}}\right)\left\{\left\langle 0\left|j_{0}(0)\right| n_{1}\left(p_{n_{1}}\right)\right\rangle\left\langle n_{1}\left(p_{n_{1}}\right)|\Phi(0)| 0\right\rangle e^{-i E_{n_{1}} x^{0}}\right. \\
\left.-\left\langle 0|\Phi(0)| n_{1}\left(p_{n_{1}}\right)\right\rangle\left\langle n_{1}\left(p_{n_{1}}\right)\left|j_{0}(0)\right| 0\right\rangle e^{i E_{n_{1}} x^{0}}\right\} & \left.-\left\langle 0|\Phi(0)| n^{\prime}\right\rangle\left\langle n^{\prime}\left|j_{0}(0)\right| 0\right\rangle e^{i E_{n^{\prime}} x^{0}}\right\},
\end{aligned}
$$

where we use the translational invariance. In the last equality, the sum of states is divided into two parts: one particle states and others. In addition, we recover the invariant measure of the integration in the part of the one-particle states. Because of the delta function and the independence of $x^{0}$, there must exist a state of $E=p^{i}=0$ except for the vacuum state. From the spectral condition of the field theory, this means the existence 
of massless one particle state. The state $|\mathrm{NG}(p)\rangle$, Nambu-Goldstone boson state, must satisfy the condition

$$
\begin{aligned}
\left\langle 0\left|j_{\mu}(0)\right| \mathrm{NG}(p)\right\rangle & \propto p_{\mu} \neq 0 \\
\langle\mathrm{NG}(p)|\Phi(0)| 0\rangle & \neq 0
\end{aligned}
$$

where we use the Lorentz covariance and the finiteness of the vacuum expectation value. This equation means that the Nambu-Goldstone boson couples to the current $j_{\mu}$. It is apparent that the number of Nambu-Goldstone bosons equals to the number of independent broken generators.

Consider an example of the Lagrangian with spontaneous symmetry breaking. The Lagrangian is the same as eq.(2.1) but the sign of the mass term is different.

$$
\mathcal{L}=\left(\partial_{\mu} \phi\right)^{\dagger}\left(\partial^{\mu} \phi\right)+m^{2} \phi^{\dagger} \phi-\lambda\left(\phi^{\dagger} \phi\right)^{2}
$$

This is invariant under the transformation of eq.(2.2). We introduce the two real fields as $\phi=(\sigma+i \pi) / \sqrt{2}$. The transformation properties of the fields $\sigma$ and $\pi$ are

$$
\begin{aligned}
& {[Q, \sigma]=i \pi} \\
& {[Q, \pi]=-i \sigma}
\end{aligned}
$$

The potential of the fields $\sigma$ and $\pi$ is

$$
V(\sigma, \pi)=-\frac{1}{2} m^{2}\left(\sigma^{2}+\pi^{2}\right)+\frac{\lambda}{4}\left(\sigma^{2}+\pi^{2}\right)^{2},
$$

and the minimum point is at $\sigma=\sqrt{m^{2} / \lambda}, \pi=0$ (We can always set $\pi=0$ by using the transformation of eq.(2.2)). This value of $\sigma$ is translated as the vacuum expectation value

$$
\langle\sigma\rangle \equiv v=\sqrt{\frac{m^{2}}{\lambda}} .
$$

The physical field $\sigma_{\text {phys }}$ which has positive squared mass is given by the shift $\sigma_{\text {phys }}=\sigma-v$. The mass of $\pi$ vanishes by this shift. The current of symmetry is written as

$$
j_{\mu}=-v \partial_{\mu} \pi+\left(\pi \partial_{\mu} \sigma_{p h y s}-\sigma_{\text {phys }} \partial_{\mu} \pi\right)
$$

by this physical field, and we can see that $\pi$ couples to the current as

$$
\left\langle 0\left|j_{\mu}(0)\right| \pi(p)\right\rangle=i p_{\mu} v \neq 0 \text {. }
$$

in the lowest order of the perturbation (Consider the fields as the asymptotic ones.). Therefore, $\pi$ is the field of Nambu-Goldstone boson.

If the local symmetry is spontaneously broken, the corresponding gauge boson becomes massive. This is called Higgs mechanism. Consider the Lagrangian of eq.(2.3) but with negative squared mass.

$$
\mathcal{L}=\left(D_{\mu} \phi\right)^{\dagger}\left(D^{\mu} \phi\right)+m^{2} \phi^{\dagger} \phi-\lambda\left(\phi^{\dagger} \phi\right)^{2}-\frac{1}{4} F_{\mu \nu} F^{\mu \nu}
$$


The Lagrangian is invariant under the transformation of eq.(2.4), but this symmetry is spontaneously broken by the vacuum expectation value of $\sigma$ just the same as the above example. By the shifting $\sigma_{\text {phys }}=\sigma-v$, the mass term of gauge boson $A_{\mu}$ is generated from the first term of the Lagrangian. The mass is controlled by the gauge coupling and the vacuum expectation value of $\sigma: m_{A}^{2}=g^{2} v^{2}$.

The complete calculation for the gauge boson mass in the second order of the gauge coupling is as follows. We rewrite the Lagrangian as

$$
\begin{aligned}
\mathcal{L} & =\mathcal{L}_{0}+g A^{\mu} j_{\mu}+g^{2} \phi^{\dagger} \phi A_{\mu} A^{\mu}, \\
\mathcal{L}_{0} & =\left(\partial_{\mu} \phi\right)^{\dagger}\left(\partial^{\mu} \phi\right)+m^{2} \phi^{\dagger} \phi-\lambda\left(\phi^{\dagger} \phi\right)^{2}-\frac{1}{4} F_{\mu \nu} F^{\mu \nu} .
\end{aligned}
$$

The generating functional is

$$
\begin{aligned}
& Z=\int \mathcal{D} A \mathcal{D} \phi \mathcal{D} \phi^{\dagger} \exp i \int d^{4} x\left\{\mathcal{L}_{0}+g A^{\mu} j_{\mu}+g^{2} \phi^{\dagger} \phi A_{\mu} A^{\mu}\right\} \\
& \simeq \int \mathcal{D} A \mathcal{D} \phi \mathcal{D} \phi^{\dagger}\left[1+i g^{2} \int_{x}\left\langle\mathrm{~T} \phi^{\dagger}(x) \phi(x)\right\rangle_{\phi} A_{\mu}(x) A^{\mu}(x)\right. \\
&\left.-\frac{1}{2} g^{2} \int_{x, y} A^{\mu}(x)\left\langle\mathrm{T} j_{\mu}(x) j_{\nu}(y)\right\rangle_{\phi} A^{\nu}(y)\right] \exp i \int d^{4} x \mathcal{L}_{0} \\
& \simeq \int \mathcal{D} A \mathcal{D} \phi \mathcal{D} \phi^{\dagger} \exp \left[i \int d^{4} x \mathcal{L}_{0}+i g^{2} \int_{x}\left\langle\mathrm{~T} \phi^{\dagger}(x) \phi(x)\right\rangle_{c, \phi} A_{\mu}(x) A^{\mu}(x)\right. \\
&\left.-\frac{1}{2} g^{2} \int_{x, y} A^{\mu}(x)\left\langle\mathrm{T} j_{\mu}(x) j_{\nu}(y)\right\rangle_{c, \phi} A^{\nu}(y)\right]
\end{aligned}
$$

where $\langle\ldots\rangle_{c, \phi}$ means the connected Green function containing only the loop effect due to the field $\phi$. We used the relation

$$
\left\langle T \mathcal{O}(x) \mathcal{O}^{\prime}(y)\right\rangle=\frac{\int \mathcal{D} A \mathcal{D} \phi \mathcal{D} \phi^{\dagger} \mathcal{O}(x) \mathcal{O}^{\prime}(y) e^{i \int d^{4} x \mathcal{L}_{0}}}{\int \mathcal{D} A \mathcal{D} \phi \mathcal{D} \phi^{\dagger} e^{i \int d^{4} x \mathcal{L}_{0}}}
$$

for any operators $\mathcal{O}(x)$ and $\mathcal{O}^{\prime}(y)$. Therefore, the effective action for the gauge field in the second order of $g$ is

$$
\begin{aligned}
i S_{e f f} & =i \int_{x, y} \frac{1}{2} A^{\mu}(x) \delta^{4}(x-y)\left(g_{\mu \nu} \square-\left(1-\frac{1}{\alpha}\right) \partial_{\mu} \partial_{\nu}\right) A^{\nu}(y) \\
& +i g^{2} \int_{x}\left\langle\mathrm{~T} \phi^{\dagger}(x) \phi(x)\right\rangle_{c, \phi} A^{\mu}(x) A_{\mu}(x) \\
& -\frac{1}{2} g^{2} \int_{x, y} A^{\mu}(x)\left\langle\mathrm{T} j_{\mu}(x) j_{\nu}(y)\right\rangle_{c, \phi} A^{\nu}(y) \\
& +\ldots,
\end{aligned}
$$

where $\alpha$ is a gauge parameter. Therefore, the propagator of gauge boson is

$$
D_{\mu \nu}(k)=\frac{1}{k^{2}-g^{2} v^{2}}\left\{g_{\mu \nu}-(1-\alpha) \frac{k_{\mu} k_{\nu}}{k^{2}}-\alpha \frac{g^{2} v^{2}}{k^{2}} \frac{k_{\mu} k_{\nu}}{k^{2}}\right\},
$$

where we use

$$
\left\langle\mathrm{T} \phi^{\dagger}(x) \phi(x)\right\rangle_{c, \phi}=\frac{v^{2}}{2}+\text { loop diagrams }
$$


and

$$
\text { F.T. }\left\langle\mathrm{T} j_{\mu}(x) j_{\nu}(y)\right\rangle_{c, \phi}=i v^{2} \frac{k_{\mu} k_{\nu}}{k^{2}}+\text { loop diagrams, }
$$

and neglect the loop effects. The propagator has a pole at $k^{2}=m_{A}^{2}=g^{2} v^{2}$. It is important that both the direct contribution of vacuum expectation value (eq.(2.37)) and the currentcurrent correlation (eq.(2.38)) contribute to the propagator. In the next chapter we treat the spontaneous symmetry breaking without the vacuum expectation value of elementary field. In that case, the contribution of current-current correlation is essential. We can see that if the massless particles couple with the currents (massless pole $1 / k^{2}$ in eq.(2.38)), the gauge bosons which couple to the current become massive (Schwinger mechanism). 


\section{Chapter 3}

\section{Dynamical Symmetry Breaking}

The symmetry of Lagrangian is spontaneously broken by the non-zero vacuum expectation value of the operator which is not invariant under the transformation of the symmetry. The operator is not always be the elementary field, but also the operator composed by the elementary fields (composite operator). The spontaneous symmetry breaking which is triggered by the vacuum expectation value of the composite operator is called dynamical symmetry breaking.

It is believed that the dynamical symmetry breaking is realized in the theory of strong interaction (quantum chromodynamics, QCD). The Lagrangian of the theory is

$$
\mathcal{L}_{Q C D}=\bar{\psi}_{i} i \not D \psi^{i}-\bar{\psi}_{i} m_{i} \psi^{i}-\frac{1}{2} \operatorname{tr}\left(F_{\mu \nu} F^{\mu \nu}\right),
$$

where

$$
\begin{aligned}
D_{\mu} \psi^{i} & =\partial_{\mu} \psi^{i}-i g G_{\mu} \psi^{i}, \\
F_{\mu \nu} & =\partial_{\mu} G_{\nu}-\partial_{\nu} G_{\mu}-i g\left[G_{\mu}, G_{\nu}\right], \\
G_{\mu} & =G_{\mu}^{a} \frac{\lambda^{a}}{2} .
\end{aligned}
$$

Here, $G_{\mu}^{a}$ denote the gluon fields ( $a=1,2, \cdots, 8$, and $\lambda^{a}$ are the Gell-Mann matrixes.) which couple with the quark fields $\psi^{i}\left(i=1,2, \cdots, N_{f}\right)$ with coupling constant $g$. If the quark masses $m_{i}$ vanish, the global chiral symmetry $S U\left(N_{f}\right)_{L} \times S U\left(N_{f}\right)_{R}$ exists. The transformation of the symmetry is

$$
\begin{array}{lll}
\psi_{L}^{i} \longrightarrow U_{L}{ }_{j} \psi_{L}^{j} & U_{L} \in S U\left(N_{f}\right)_{L}, \\
\psi_{R}^{i} \longrightarrow U_{R}{ }_{j} \psi_{R}^{j} & U_{R} \in S U\left(N_{f}\right)_{R} .
\end{array}
$$

As long as we consider the light (compared with the typical scale of QCD dynamics) quarks, u-quark and d-quark (and s-quark), this is a good approximate symmetry. It is believed that this chiral symmetry is dynamically broken to the symmetry $S U\left(N_{f}\right)_{V}$

by the quark pair condensation $\left\langle\bar{\psi}_{i} \psi^{i}\right\rangle \neq 0$ due to the QCD dynamics. The composite operator $\bar{\psi}_{i} \psi^{i}$ is not invariant under the chiral symmetry but invariant under the $S U\left(N_{f}\right)_{V}$ transformation

$$
\psi^{i} \longrightarrow U^{i}{ }_{j} \psi^{j} \quad U \in S U\left(N_{f}\right)_{V} .
$$


If we consider $\mathrm{u}$ and d quarks ( $\mathrm{u}, \mathrm{d}$, and s quarks) $\left(N_{f}=2(3)\right)$ and neglect their masses (chiral limit), there should be three (eight) pseudo-scalar massless Nambu-Goldstone bosons. The pseudo-scalar mesons, $\pi^{0}$ and $\pi^{ \pm}\left(\pi^{0}, \pi^{ \pm}, K^{0}, \bar{K}^{0}, K^{ \pm}\right.$, and " $\eta_{8}$ "), can be identified as the Nambu-Goldstone bosons. The masses of these mesons are understood as the effect of the explicit chiral symmetry breaking due to the quark masses. The effect can be perturbatively included, if the masses of the quarks are small enough (chiral perturbation) 13. The interactions between these mesons at low energy (low energy $\pi-\pi$ scattering, for example) are described as the interactions of the Nambu-Goldstone bosons which are determined only by the symmetry (low energy theorems). The correction due to the explicit breaking is perturbatively considered. The fact that the picture of the chiral perturbation can satisfactory describe the low energy phenomenology of QCD is the reason why we believe the existence of the pair condensation in QCD.

In next section, we try to calculate the value of the pair condensation in QCD by using Schwinger-Dyson equation 14 and operator product expansion [15]. The Schwinger-Dyson equation (self-consistent equation for quark propagator) is solved in ladder approximation, and the non-trivial solution is converted into the pair condensation $\left\langle\bar{\psi}_{i} \psi^{i}\right\rangle$ by using the operator product expansion. In section 2, we calculate the decay constant of NambuGoldstone boson which corresponds to the vacuum expectation value of the elementary scalar field in the previous chapter. The Pagels-Stokar formula[16], which gives decay constant as a functional of the quark propagator, is introduced there. In section 3, we discuss the Cornwall-Jackiw-Tomboulis effective action [17 by which the general treatment of spontaneous symmetry breaking is possible.

\subsection{Schwinger-Dyson equation and quark condensa- tion}

We consider the Schwinger-Dyson equation 14 for the quark and gluon propagators. We want to solve the equation, and get the full quark propagator. The equation is

$$
\begin{aligned}
& i S^{-1}(x-y)^{i}{ }_{j} \delta_{\beta}^{\alpha}=\left(i \not \partial-m_{i}\right) \delta_{j}^{i} \delta_{\beta}^{\alpha} \delta^{4}(x-y) \\
&+g^{2} \int d^{4} x_{1} d^{4} x_{2} \gamma^{\mu}\left(T^{a}\right)^{\alpha}{ }_{\gamma} S\left(x-x_{1}\right)^{i}{ }_{k} \Gamma^{\nu}\left(x_{2}, x_{1}, y\right)^{k}{ }_{j}\left(T^{a}\right)^{\gamma}{ }_{\beta} D_{\mu \nu}\left(x-x_{2}\right), \\
& D_{\mu \nu}^{-1}(x-y) \delta^{a b}=-i\left\{\square g_{\mu \nu}-\left(1-\frac{1}{\alpha}\right) \partial_{\mu} \partial_{\nu}\right\} \delta^{a b} \delta^{4}(x-y) \\
&+i g^{2} \int d^{4} x_{1} d^{4} x_{2} \operatorname{tr}\left[\gamma_{\mu} T^{a} S\left(x-x_{1}\right) \Gamma_{\nu}\left(y, x_{1}, x_{2}\right) T^{b} S\left(x_{2}-x\right)\right],
\end{aligned}
$$

where $S$ and $D$ are the full propagators (bare) of quarks and gluons

$$
\begin{aligned}
S(x-y)^{i}{ }_{j} \delta_{\beta}^{\alpha} & \equiv-\left.\frac{\delta^{2} \ln Z}{\delta i \bar{\eta}^{i \alpha}(x) \delta i \eta_{j \beta}(y)}\right|_{J, \eta, \bar{\eta}=0}, \\
D_{\mu \nu}(x-y) \delta^{a b} & \left.\equiv \frac{\delta^{2} \ln Z}{\delta i J^{\mu a}(x) \delta i J^{\nu b}(y)}\right|_{J, \eta, \bar{\eta}=0},
\end{aligned}
$$


(The left-derivative of the Grassmann variable is understood.) and $\Gamma$ is a full gluon-quarkquark vertex (bare)

$$
\begin{aligned}
& \left.\frac{\delta^{3} \ln Z}{\delta i J^{\mu a}(x) \delta i \bar{\eta}^{i \alpha} \delta i \eta_{j \beta}}\right|_{J, \eta, \bar{\eta}=0} \\
& \equiv-\int d^{4} x^{\prime} d^{4} y^{\prime} d^{4} z^{\prime} D_{\mu \nu}\left(x-z^{\prime}\right) S\left(x-x^{\prime}\right)^{i}{ }_{k} g \Gamma^{\nu}\left(z^{\prime}, x^{\prime}, y^{\prime}\right)^{k}{ }_{l}\left(T^{a}\right)^{\beta}{ }_{\alpha} S\left(y^{\prime}-y\right)^{l}{ }_{j} .
\end{aligned}
$$

The matrixes $T^{a}$ are the generators of $S U(3)_{c}\left(T^{a}=\lambda^{a} / 2\right)$. The indexes $i, j, k$ denote flavor, $\alpha, \beta, \gamma$ denote the color of quarks, and $a, b$ denote the color of gluons. We are assuming that the gauge symmetry $S U(3)_{c}$ is not broken. The propagators of the quarks and gluons are set to diagonal in the color space. The functional $Z$ is the generating functional of the Green functions

$$
\begin{gathered}
Z[J, \eta, \bar{\eta}]=\int \mathcal{D}(G, \psi, \bar{\psi}) \exp i S[G, \psi, \bar{\psi}, J, \eta, \bar{\eta}] \\
S[G, \psi, \bar{\psi}, J, \eta, \bar{\eta}]=\int d^{4} x\left[\mathcal{L}_{Q C D}+J_{\mu} G^{\mu}+\bar{\eta} \psi+\bar{\psi} \eta\right],
\end{gathered}
$$

where $J_{\mu}, \eta$, and $\bar{\eta}$ are the source fields.

We can derive eq.(3.8) as follows. The equation

$$
\int \mathcal{D}(G, \psi, \bar{\psi}) \frac{\delta S[G, \psi, \bar{\psi}, J, \eta, \bar{\eta}]}{\delta \bar{\psi}^{i \alpha}(x)} \exp i S[G, \psi, \bar{\psi}, J, \eta, \bar{\eta}]=0
$$

is satisfied as the field equation of quarks. This equation means that the expectation value of the derivative of the action by quarks should vanish. It is rewritten as

$$
\left[\eta(x)^{i \alpha}+\left(\left(i \not \partial_{x}-m_{i}\right) \delta_{\gamma}^{\alpha}+g \gamma^{\mu}\left(T^{a}\right)^{\alpha} \frac{\delta}{\delta i J^{a \mu}(x)}\right) \frac{\delta}{\delta i \bar{\eta}^{i \gamma}(x)}\right] Z[j, \eta, \bar{\eta}]=0 .
$$

Differentiating with respect to $\eta^{j \beta}(y)$ and dividing by $Z$ give

$$
\begin{aligned}
& \delta^{4}(x-y) \delta_{j}^{i} \delta_{\beta}^{\alpha} \\
& +\left(i \not \partial_{x}-m_{i}\right) \delta_{\gamma}^{\alpha} i \frac{1}{Z} \frac{\delta^{2} Z}{\delta \bar{\eta}^{i \gamma}(x) \delta \eta_{j \beta}(y)}+g \gamma^{\mu}\left(T^{a}\right)^{\alpha}{ }_{\gamma} \frac{1}{Z} \frac{\delta}{\delta J^{a \mu}(x)} \frac{\delta^{2} Z}{\delta \bar{\eta}^{i \gamma}(x) \delta \eta_{j \beta}(y)}=0 \\
& \delta^{4}(x-y) \delta_{j}^{i} \delta_{\beta}^{\alpha} \\
& +\left(i \partial_{x}-m_{i}\right) \delta_{\gamma}^{\alpha} i\left(\frac{\delta^{2} \ln Z}{\delta \bar{\eta}^{i \gamma}(x) \delta \eta_{j \beta}(y)}+\frac{\delta \ln Z}{\delta \bar{\eta}^{i \gamma}(x)} \frac{\delta \ln Z}{\delta \eta_{j \beta}(y)}\right) \\
& +g \gamma^{\mu}\left(T^{a}\right)^{\alpha}{ }_{\gamma}\left(\frac{\delta^{3} \ln Z}{\delta J^{a \mu}(x) \delta \bar{\eta}^{i \gamma}(x) \delta \eta_{j \beta}(y)}+\frac{\delta}{\delta J^{a \mu}(x)}\left(\frac{\delta \ln Z}{\delta \bar{\eta}^{i \gamma}(x)} \frac{\delta \ln Z}{\delta \eta_{j \beta}(y)}\right)\right. \\
& \left.\quad+\frac{\delta \ln Z}{\delta J^{a \mu}(x)}\left(\frac{\delta^{2} \ln Z}{\delta \bar{\eta}^{i \gamma}(x) \delta \eta_{j \beta}(y)}+\frac{\delta \ln Z}{\delta \bar{\eta}^{i \gamma}(x)} \frac{\delta \ln Z}{\delta \eta_{j \beta}(y)}\right)\right)=0
\end{aligned}
$$


By setting $J, \bar{\eta}, \eta=0$, and assuming

$$
\begin{aligned}
\left.\frac{\delta \ln Z}{\delta J^{a \mu}(x)}\right|_{J, \eta, \bar{\eta}=0} & =\left.i\left\langle G_{\mu}^{a}\right\rangle\right|_{J, \eta, \bar{\eta}=0}=0, \\
\left.\frac{\delta \ln Z}{\delta \bar{\eta}^{i \alpha}(x)}\right|_{J, \eta, \bar{\eta}=0} & =\left.i\left\langle\psi_{i \alpha}(x)\right\rangle\right|_{J, \eta, \bar{\eta}=0}=0, \\
\left.\frac{\delta \ln Z}{\delta \eta_{i \alpha}(x)}\right|_{J, \eta, \bar{\eta}=0} & =-\left.i\left\langle\bar{\psi}^{i \alpha}(x)\right\rangle\right|_{J, \eta, \bar{\eta}=0}=0,
\end{aligned}
$$

we obtain

$$
\begin{aligned}
& \delta^{4}(x-y) \delta_{j}^{i} \delta_{\beta}^{\alpha} \\
& \quad+\left.\left(i \not \partial_{x}-m_{i}\right) \delta_{\gamma}^{\alpha} i \frac{\delta^{2} \ln Z}{\delta \bar{\eta}^{i \gamma}(x) \delta \eta_{j \beta}(y)}\right|_{J, \eta, \bar{\eta}=0} \\
& \quad+\left.g \gamma^{\mu}\left(T^{a}\right)^{\alpha}{ }_{\gamma} \frac{\delta^{3} \ln Z}{\delta J^{a \mu}(x) \delta \bar{\eta}^{i \gamma}(x) \delta \eta_{j \beta}(y)}\right|_{J, \eta, \bar{\eta}=0}=0 .
\end{aligned}
$$

From the definition of $S$ and $\Gamma$ and

$$
\int d^{4} z S(x-z)_{\gamma k}^{\alpha} S^{-1}(z-y)^{\gamma}{ }^{k}{ }_{j}=\delta_{\beta}^{\alpha} \delta_{j}^{i} \delta^{4}(x-y)
$$

we get the Schwinger-Dyson equation for quark propagator eq.(3.8). The Schwinger-Dyson equation for gluon propagator, eq.(3.10), can also be obtained in the same way.

The eq.(3.8) and eq.(3.10) can be written as follows in momentum space.

$$
\begin{aligned}
& S^{-1}(k)=(m-\not k)-g^{2} C_{2} \int \frac{d^{4} q}{(2 \pi)^{4} i} \gamma^{\mu} S(q) \Gamma^{\nu}(k-q, q,-k) D_{\mu \nu}(k-q) \\
& D_{\mu \nu}^{-1}(k)=\left\{k^{2} g_{\mu \nu}-\left(1-\frac{1}{\alpha}\right) k_{\mu} k_{\nu}\right\} \\
&-i g^{2} C_{2} \int \frac{d^{4} q}{(2 \pi)^{4} i} \operatorname{tr}\left[\gamma_{\mu} S(q) \Gamma_{\nu}(-k, q, k-q) S(q-k)\right]
\end{aligned}
$$

where $C_{2}=\operatorname{tr}\left(T^{a} T^{a}\right) / N_{c}\left(N_{c}=3\right), m=\operatorname{diag}\left(m_{1}, m_{2}, \cdots, m_{N_{f}}\right)$,

$$
\begin{aligned}
S(k) & =(\Sigma(k)-\not k)^{-1}, \\
S^{-1}(k) & =\Sigma(k)-\not k \\
S(x-y) & =\int \frac{d^{4} q}{(2 \pi)^{4} i} S(q) e^{-i q(x-y)}, \\
S^{-1}(x-y) & =\int \frac{d^{4} q}{(2 \pi)^{4}} i S^{-1}(q) e^{-i q(x-y)},
\end{aligned}
$$




$$
\begin{aligned}
D_{\mu \nu}(k) & =\frac{1}{k^{2}\left(1+\Pi\left(k^{2}\right)\right)}\left(g_{\mu \nu}-(1-\alpha) \frac{k_{\mu} k_{\nu}}{k^{2}}\right), \\
D_{\mu \nu}^{-1}(k) & =\left(1+\Pi\left(k^{2}\right)\right)\left(k^{2} g_{\mu \nu}-\left(1-\frac{1}{\alpha}\right) k_{\mu} k_{\nu}\right), \\
D_{\mu \nu}(x-y) & =\int \frac{d^{4} q}{(2 \pi)^{4} i} D_{\mu \nu}(q) e^{-i q(x-y)}, \\
D_{\mu \nu}^{-1}(x-y) & =\int \frac{d^{4} q}{(2 \pi)^{4}} i D_{\mu \nu}^{-1}(q) e^{-i q(x-y)},
\end{aligned}
$$

and

$$
\begin{aligned}
\Gamma^{\mu}\left(y, x_{1}, x_{2}\right) \\
\quad=\int \frac{d^{4} q}{(2 \pi)^{4}} \frac{d^{4} p_{1}}{(2 \pi)^{4}} \frac{d^{4} p_{2}}{(2 \pi)^{4}}(2 \pi)^{4} i \delta^{4}\left(q+p_{1}+p_{2}\right) \Gamma^{\mu}\left(q, p_{1}, p_{2}\right) e^{-i q y} e^{-i p_{1} x_{1}} e^{-i p_{2} x_{2}} .
\end{aligned}
$$

All the quantities in eqs.(3.23) and (3.24) are bare ones. The Schwinger-Dyson equation for renormalized quantities is

$$
\begin{gathered}
S_{R}^{-1}(k)=Z_{2}\left(\frac{m_{R}+\delta m}{Z_{2}}-\not k\right) \\
-Z_{1} g_{R}^{2} C_{2} \int \frac{d^{4} q}{(2 \pi)^{4} i} \gamma^{\mu} S_{R}(q) \Gamma_{R}^{\nu}(k-q, q,-k) D_{R \mu \nu}(k-q), \\
D_{R \mu \nu}^{-1}(k)=Z_{3}\left(k^{2} g_{\mu \nu}-\left(1-\frac{1}{\alpha}\right) k_{\mu} k_{\nu}\right) \\
-i Z_{1} g_{R}^{2} C_{2} \int \frac{d^{4} q}{(2 \pi)^{4} i} \operatorname{tr}\left[\gamma_{\mu} S_{R}(q) \Gamma_{R \nu}(-k, q, k-q) S_{R}(q-k)\right] .
\end{gathered}
$$

Z-factors and $\delta m_{i}$ should be fixed by the renormalization conditions, for example,

$$
\begin{gathered}
\left.\frac{1}{4} \operatorname{tr} S_{R}^{-1}(k)\right|_{k^{2}=\mu^{2}}=\mu, \\
\left.\frac{\partial}{\partial k^{2}} \frac{1}{4} \operatorname{tr} \not k S_{R}^{-1}(k)\right|_{k^{2}=\mu^{2}}=-1 .
\end{gathered}
$$

Now we solve the equation. We consider the case that the chiral symmetry is exact, $m_{i}=0$. The flavor dependence of the propagator will disappear, and the global $S U\left(N_{f}\right)_{V}$ symmetry will not be broken 1 . Take the full vertex function (bare) as

$$
\Gamma^{\mu}\left(q, p_{1}, p_{2}\right)^{i}{ }_{j}=\gamma^{\mu} \delta_{j}^{i},
$$

and take the gluon propagator in eq.(3.23) as free one. Then the equation becomes

$$
S^{-1}(k)=-\not k-g^{2} C_{2} \int \frac{d^{4} q}{(2 \pi)^{4} i} \gamma^{\mu} S(q) \gamma^{\nu} D_{\mu \nu}^{\text {free }}(k-q)
$$

\footnotetext{
${ }^{1}$ We simply assume this.
} 


$$
D_{\mu \nu}^{\text {free }}(k)=\frac{1}{k^{2}}\left\{g_{\mu \nu}-(1-\alpha) \frac{k_{\mu} k_{\nu}}{k^{2}}\right\},
$$

where we set $S(q)_{j}^{i}=S(q) \delta_{j}^{i}$. This approximation is called ladder approximation. The equation of the quark propagator is independent of the equation of the gluon propagator. Though there is no reason for supporting this approximation, it is taken only for the analytic treatment of the equation. Since the result is gauge dependent ( $\alpha$ dependent), we can not do the quantitative discussion. We believe that this approximation does not destroy the qualitative feature.

The quark propagator can be generally written as

$$
S^{-1}(k)=B\left(k^{2}\right)-A\left(k^{2}\right) \not k .
$$

We get two equation for $A\left(k^{2}\right)$ and $B\left(k^{2}\right)$ from eq.(3.39). By taking trace, we get

$$
B\left(k^{2}\right)=-\frac{1}{4} g^{2} C_{2} \operatorname{tr} \int \frac{d^{4} q}{(2 \pi)^{4} i} \gamma^{\mu} S(q) \gamma^{\nu} D_{\mu \nu}^{\text {free }}(k-q),
$$

and by taking trace after multiplying $-\not k / k^{2}$, we get

$$
A\left(k^{2}\right)=1+\frac{1}{4} g^{2} C_{2} \operatorname{tr} \frac{\not k}{k^{2}} \int \frac{d^{4} q}{(2 \pi)^{4} i} \gamma^{\mu} S(q) \gamma^{\nu} D_{\mu \nu}^{\text {free }}(k-q) .
$$

In the Euclidean space 2, they become

$$
\begin{gathered}
B\left(-k^{2}\right)=\frac{3+\alpha}{3} \lambda \int d q^{2} \frac{q^{2} B\left(-q^{2}\right)}{B\left(-q^{2}\right)^{2}+A\left(-q^{2}\right)^{2} q^{2}}\left\{\frac{1}{k^{2}} \theta\left(k^{2}-q^{2}\right)+\frac{1}{q^{2}} \theta\left(q^{2}-k^{2}\right)\right\} \\
A\left(-k^{2}\right)=1+\frac{\alpha}{3} \lambda \frac{1}{k^{2}} \int d q^{2} \frac{q^{2} A\left(-q^{2}\right)}{B\left(-q^{2}\right)^{2}+A\left(-q^{2}\right)^{2} q^{2}}\left\{\frac{1}{k^{2}} \theta\left(k^{2}-q^{2}\right)+\frac{1}{q^{2}} \theta\left(q^{2}-k^{2}\right)\right\}
\end{gathered}
$$

where

$$
\lambda=\frac{3}{4 \pi} C_{2} \frac{g^{2}}{4 \pi}
$$

If we take Landau gauge $\alpha=0$ ㅇ, $A\left(-k^{2}\right)=1$ and

$$
B\left(-k^{2}\right)=\lambda \int d q^{2} \frac{q^{2} B\left(-q^{2}\right)}{B\left(-q^{2}\right)^{2}+q^{2}}\left\{\frac{1}{k^{2}} \theta\left(k^{2}-q^{2}\right)+\frac{1}{q^{2}} \theta\left(q^{2}-k^{2}\right)\right\} .
$$

This non-linear equation can not be solved analytically. We use the bifurcation theory 18] to estimate the existence of the non-trivial solution. We expand the non-linear equation around the trivial solution as

$$
\Sigma(x)=\lambda \int y \Sigma(y)\left\{\frac{1}{y} \theta(y-x)+\frac{1}{x} \theta(x-y)\right\},
$$

\footnotetext{
${ }^{2}$ It is a non-trivial problem whether we can go to the Euclidean space or not. Here, we simply assume that we can do that.

${ }^{3}$ When we consider QED, to take Landau gauge makes the ladder approximation consistent with the Ward-Takahashi identity.
} 
where $\Sigma(x)=B\left(-k^{2}\right)$ with $x=k^{2}$ and $y=q^{2} . \Sigma(x)$ is called the mass function. Bifurcation theory says that if this expanded equation has only one non-trivial linearly independent solution when $\lambda=\lambda_{c}$, the full equation has only one non-trivial solution (up to sign) when $\lambda>\lambda_{c}$ or $\lambda<\lambda_{c}$. The linearized equation can be transformed to the differential equation with boundary conditions as

$$
\begin{gathered}
x^{2} \frac{d^{2} \Sigma(x)}{d x^{2}}+2 x \frac{d \Sigma(x)}{d x}+\lambda \Sigma(x)=0, \\
\lim _{x \rightarrow 0} x^{2} \Sigma^{\prime}(x)=0, \\
\lim _{x \rightarrow \infty}(x \Sigma(x))^{\prime}=0 .
\end{gathered}
$$

This can be easily solved. The general two linearly independent non-trivial solutions are

$$
\Sigma(x)=\left\{\begin{array}{l}
x^{-(1-\gamma) / 2} \\
x^{-(1+\gamma) / 2}
\end{array}\right.
$$

where $\gamma=\sqrt{1-4 \lambda}$. These solutions satisfy the boundary conditions if $\lambda \neq 0$. When $\lambda=\lambda_{c} \equiv 1 / 4$, the two solutions are not linearly independent. Therefore, we know that the non-linear equation has only one non-trivial solution (up to sign) when $\lambda>\lambda_{c}$ or $\lambda<\lambda_{c} . \lambda_{c}$ is called the critical coupling.

The non-linear equation is expected to have the non-trivial solution when $\lambda>\lambda_{c}$ [19]. By replacing the $B\left(-q^{2}\right)=\Sigma(y)$ in the denominator of eq.(3.47) into the constant $m$ $(m=\Sigma(m))$, we get

$$
\Sigma(x)=\lambda \int d y \frac{y \Sigma(y)}{m^{2}+y}\left\{\frac{1}{x} \theta(x-y)+\frac{1}{y} \theta(y-x)\right\} .
$$

This equation can be transformed to the differential equation and boundary conditions as

$$
\begin{gathered}
(x \Sigma(x))^{\prime \prime}+\frac{\lambda \Sigma(x)}{x+m^{2}}=0, \\
\lim _{x \rightarrow 0} x^{2} \Sigma^{\prime}(x)=0, \\
\lim _{x \rightarrow \Lambda^{2}}(x \Sigma(x))^{\prime}=0,
\end{gathered}
$$

where $\Lambda$ is the ultraviolet cut off. By changing the valuable $x$ to $y=-x / m^{2}$, we get

$$
y(1-y) \frac{d^{2} \Sigma}{d y^{2}}+2(1-y) \frac{d \Sigma}{d y}-\lambda \Sigma=0 .
$$

This equation is called hypergeometric differential equation which can be solved analytically. The general solution is

$$
\begin{aligned}
\Sigma(x)= & a_{1} F\left(\frac{1}{2}+\gamma, \frac{1}{2}-\gamma, 2 ;-\frac{x}{m^{2}}\right) \\
& -a_{2}\left(-\frac{x}{m^{2}}\right)^{-1} F\left(-\frac{1}{2}+\gamma,-\frac{1}{2}-\gamma, 0 ;-\frac{x}{m^{2}}\right)
\end{aligned}
$$


for $x / m^{2}<1$, and

$$
\begin{aligned}
\Sigma(x)= & b_{1}\left(\frac{m^{2}}{x}\right)^{\frac{1+\gamma}{2}} F\left(\frac{1+\gamma}{2},-\frac{1-\gamma}{2}, 1+\gamma ;-\frac{m^{2}}{x}\right) \\
& +b_{2}\left(\frac{m^{2}}{x}\right)^{\frac{1-\gamma}{2}} F\left(\frac{1-\gamma}{2},-\frac{1+\gamma}{2}, 1-\gamma ;-\frac{m^{2}}{x}\right)
\end{aligned}
$$

for $m^{2} / x<1$, where $a_{1}, a_{2}, b_{1}$, and $b_{2}$ are the constants. The function $F$ is hypergeometric series

$$
F(\alpha, \beta, \gamma ; z) \equiv \frac{\Gamma(\gamma)}{\Gamma(\alpha) \Gamma(\beta)} \sum_{n=0}^{\infty} \frac{\Gamma(\alpha+n) \Gamma(\beta+n)}{\Gamma(\gamma+n)} \frac{z^{n}}{n !}
$$

From the infrared boundary condition, eq. (3.55), $a_{2}$ must be zero. The value $\Sigma(x=$ $0)=a_{1}$ is finite unlike the solution when $m=0$ (eq.(3.52)). The ultraviolet boundary condition eq.(3.56) is not satisfied unless $\lambda>\lambda_{c}$. The non-trivial solution which satisfy the boundary condition is

$$
\Sigma(x)=a F\left(\frac{1}{2}+i \gamma^{\prime}, \frac{1}{2}-i \gamma^{\prime}, 2 ;-\frac{x}{m^{2}}\right)
$$

for $x / m^{2}<1$, and

$$
\Sigma(x)=\frac{1}{2} b\left(\frac{m^{2}}{x}\right)^{\frac{1+i \gamma^{\prime}}{2}} F\left(\frac{1+i \gamma^{\prime}}{2},-\frac{1-i \gamma^{\prime}}{2}, 1+i \gamma^{\prime} ;-\frac{m^{2}}{x}\right)+\text { h.c. }
$$

for $m^{2} / x<1$, where $a$ and $b$ are real and complex constants, respectively, and $\gamma^{\prime}=$ $\sqrt{4 \lambda-1}>0$. We are assuming that $\Sigma(x)$ is the real function. The asymptotic form for $x \rightarrow \Lambda^{2}$ is

$$
\Sigma(x)=b\left(\frac{m^{2}}{x}\right)^{1 / 2} \cos \left(\frac{\gamma^{\prime}}{2} \ln \left(\frac{m^{2}}{x}\right)\right) .
$$

$\Sigma(x)$ dumps as $1 / \sqrt{x}$.

Next, we connect the solution of the Schwinger-Dyson equation to the pair condensate of quark $\left\langle\bar{\psi}_{i} \psi^{i}\right\rangle$. We use the technique of operator product expansion 15]. The quark propagator is expanded as

$$
\begin{aligned}
\int d^{4} x e^{i p x}\left\langle 0\left|T \psi_{L}^{i}(x) \bar{\psi}_{R}^{j}(0)\right| 0\right\rangle & =U_{\mathrm{unit}}^{i j}(p, g, \mu) \frac{1-\gamma_{5}}{2} \\
& +U_{\bar{\psi} \psi}^{i j}(p, g, \mu) \frac{1-\gamma_{5}}{2}\left\langle 0\left|T \bar{\psi}_{R}(0) \psi_{L}(0)\right| 0\right\rangle \\
& + \text { (higher dimensional operators) }
\end{aligned}
$$

where $\mu$ is the renormalization point. The first term in right-hand side is the effect of the explicit chiral symmetry breaking by which the chirality is flipped without the vacuum expectation value of operators. Since we are neglecting the explicit breaking now,

$$
\lim _{p \rightarrow \infty} \int d^{4} x e^{i p x}\left\langle 0\left|T \psi_{L}^{i}(x) \bar{\psi}_{R}^{j}(0)\right| 0\right\rangle=\lim _{p \rightarrow \infty} U_{\bar{\psi} \psi}^{i j}(p, g, \mu) \frac{1-\gamma_{5}}{2}\left\langle 0\left|\bar{\psi}_{R}(0) \psi_{L}(0)\right| 0\right\rangle .
$$


Figure 3.1: Tree diagram for four-point Green function.

Figure 3.2: Tree diagram for two point Green function with composite operator.

We can calculate the asymptotic form of the Wilson coefficient function $U_{\bar{\psi} \psi}^{i j}$ by the perturbation theory. If the operator expansion is good, the relation

$$
\begin{aligned}
& \lim _{p \rightarrow \infty} \int d^{4} x d^{4} y d^{4} z e^{i p x} e^{i q y} e^{i k z}\left\langle 0\left|T \psi_{L}^{i}(x)_{\alpha} \bar{\psi}_{R}^{j}(0)_{\delta} \psi_{R}^{k}(y)_{\gamma} \bar{\psi}_{L}^{l}(z)_{\beta}\right| 0\right\rangle \\
& =\lim _{p \rightarrow \infty} U_{\bar{\psi} \psi}^{i j}(p, g, \mu)\left(\frac{1-\gamma_{5}}{2}\right)_{\alpha \delta} \int d^{4} y d^{4} z e^{i q y} e^{i k z}\left\langle 0\left|T\left(\bar{\psi}_{R}(0) \psi_{L}(0)\right) \psi_{R}^{k}(y)_{\gamma} \bar{\psi}_{L}^{l}(z)_{\beta}\right| 0\right\rangle
\end{aligned}
$$

should be satisfied. The Green functions can be calculated in tree level as (fig.(3.1))

$$
\begin{aligned}
\lim _{p \rightarrow \infty} & \int d^{4} x d^{4} y d^{4} z e^{i p x} e^{i q y} e^{i k z}\left\langle 0\left|T \psi_{L}^{i}(x)_{\alpha} \bar{\psi}_{R}^{j}(0)_{\delta} \psi_{R}^{k}(y)_{\gamma} \bar{\psi}_{L}^{l}(z)_{\beta}\right| 0\right\rangle \\
= & -(2 \pi)^{4} \delta^{4}(k+p)\left(\frac{1-\gamma_{5}}{2} \frac{i}{\not p}\right)_{\alpha \beta}\left(\frac{1+\gamma_{5}}{2} \frac{i}{\not d}\right)_{\gamma \delta} \delta^{i l} \delta^{k j} \\
& -\left(\frac{1-\gamma_{5}}{2} \frac{i}{\not p} \gamma_{\mu} \frac{i}{-\not k}\right)_{\alpha \beta} g^{2}\left(T^{a}\right)^{i l}\left(T^{a}\right)^{k j} D^{\mu \nu}(p+k)\left(\frac{1+\gamma_{5}}{2} \frac{i}{\not q} \gamma_{\nu} \frac{i}{\not q+\not p+\not k}\right)_{\gamma \delta}
\end{aligned}
$$

and (fig.(3.2))

$$
\begin{gathered}
\int d^{4} y d^{4} z e^{i q y} e^{i k z}\left\langle 0\left|T\left(\bar{\psi}_{R}(0) \psi_{L}(0)\right) \psi_{R}^{k}(y)_{\gamma} \bar{\psi}_{L}^{l}(z)_{\beta}\right| 0\right\rangle \\
=\left(\left(\frac{1+\gamma_{5}}{2} \frac{i}{\not q}\right)\left(\frac{1-\gamma_{5}}{2} \frac{i}{-\not k}\right)\right)_{\gamma \beta} \delta^{k l}
\end{gathered}
$$

where $D^{\mu \nu}$ is the gluon free propagator

$$
D^{\mu \nu}=\frac{1}{i} \frac{1}{q^{2}}\left\{g^{\mu \nu}-(1-\alpha) \frac{q^{\mu} q^{\nu}}{q^{2}}\right\} .
$$

Therefore, when $k=-q$,

$$
\begin{gathered}
\lim _{p \rightarrow \infty}\left(\frac{1-\gamma_{5}}{2} \frac{i}{\not p} \gamma_{\mu} \frac{i}{\not 1}\right)_{\alpha \beta} g^{2}\left(T^{a}\right)^{i l}\left(T^{a}\right)^{k j} D^{\mu \nu}(p-q)\left(\frac{1+\gamma_{5}}{2} \frac{i}{\not q} \gamma_{\nu} \frac{i}{\not p}\right)_{\gamma \delta} \\
=-\lim _{p \rightarrow \infty} U_{\bar{\psi} \psi}^{i j}(p, g, \mu)\left(\frac{1-\gamma_{5}}{2}\right)_{\alpha \delta}\left(\frac{1+\gamma_{5}}{2} \frac{i}{\not q} \frac{i}{\not d}\right)_{\gamma \beta} \delta^{k l} .
\end{gathered}
$$

By taking trace for the indexes $\gamma \beta, \alpha \delta$, and $k l$, we get

$$
\lim _{p \rightarrow \infty} U_{\bar{\psi} \psi}^{i j}(p, g, \mu)=\frac{1}{i} \frac{3+\alpha}{2 N_{c}} g^{2} C_{2} \delta^{i j} \frac{1}{p^{4}},
$$

where $N_{c}$ is the color degrees of freedom of quarks $\left(N_{c}=3\right)$. 
This result is improved by using the renormalization group equation. The improvement makes it to depend on the renormalization point $\mu$. The Wilson coefficient function satisfies the following renormalization group equation.

$$
U_{\bar{\psi} \psi}^{i j}(\kappa p, g, \mu)=\kappa^{d_{U}} U_{\bar{\psi} \psi}^{i j}(p, g(\kappa), \mu) \exp \int_{1}^{\kappa}\left[\gamma_{\bar{\psi} \psi}\left(g\left(\kappa^{\prime}\right)\right)-\gamma_{\psi \bar{\psi}}\left(g\left(\kappa^{\prime}\right)\right)\right] \frac{d \kappa^{\prime}}{\kappa^{\prime}},
$$

where $d_{U}=-4$ is the dimension of the Wilson coefficient function, $\gamma_{\bar{\psi} \psi}$ and $\gamma_{\psi \bar{\psi}}$ are the anomalous dimensions of the operator $\bar{\psi} \psi$ and $T \psi \bar{\psi}$, respectively, and $g(\kappa)$ is the running coupling. This equation means the fact that the renormalization point dependence of the both side of eq. (3.64) should be the same. We multiply the function $f\left(p^{2} / \mu^{2}\right)$ to the Wilson coefficient given by the perturbative calculation to include the renormalization point dependence. By substituting it into the renormalization group equation in $p \rightarrow 0$ limit, we get

$$
\begin{aligned}
& \lim _{k \rightarrow \infty} U_{\bar{\psi} \psi}^{i j}(k, g, \mu) \\
& \quad=\left(\frac{k^{2}}{p^{2}}\right)^{d_{U} / 2} \frac{1}{i} \frac{3+\alpha}{2 N_{c}} g^{2}\left(\left(k^{2} / p^{2}\right)^{1 / 2}\right) C_{2} \delta^{i j} \frac{1}{p^{4}} f\left(p^{2} / \mu^{2}\right) \exp \int_{1}^{\left(k^{2} / p^{2}\right)^{1 / 2}} \gamma\left(g\left(\kappa^{\prime}\right)\right) \frac{d \kappa^{\prime}}{\kappa^{\prime}},
\end{aligned}
$$

where $k=\kappa p$ and

$$
\gamma\left(g\left(\kappa^{\prime}\right)\right)=\gamma_{\bar{\psi} \psi}\left(g\left(\kappa^{\prime}\right)\right)-\gamma_{\psi \bar{\psi}}\left(g\left(\kappa^{\prime}\right)\right) .
$$

The function $f\left(p^{2} / \mu^{2}\right)$, is specified by assuming that the right-hand side is only depend on $k$ but not $p$ explicitly. Namely,

$$
f\left(p^{2} / \mu^{2}\right) g^{2}\left(\left(k^{2} / p^{2}\right)^{1 / 2}\right) \exp \int_{1}^{\left(k^{2} / p^{2}\right)^{1 / 2}} \gamma\left(g\left(\kappa^{\prime}\right)\right) \frac{d \kappa^{\prime}}{\kappa^{\prime}}=\text { constant. }
$$

If the running of the coupling is very slow, we can replace it by a constant $g^{*}$. Then

$$
f\left(p^{2} / \mu^{2}\right) g^{* 2}\left(\frac{k^{2}}{p^{2}}\right)^{\frac{\gamma^{*}}{2}}=\text { constant }
$$

where $\gamma^{*}=\gamma\left(g^{*}\right)$. Therefore,

$$
f\left(p^{2} / \mu^{2}\right)=c\left(\frac{p^{2}}{\mu^{2}}\right)^{\gamma^{*} / 2}
$$

with a constant $c$. In this case, we can explicitly write the asymptotic form of the Wilson coefficient function as

$$
\lim _{k \rightarrow \infty} U_{\bar{\psi} \psi}^{i j}(k, g, \mu)=c \frac{1}{i} \frac{3+\alpha}{2 N_{c}} g^{* 2} C_{2} \delta^{i j} \frac{1}{k^{4}}\left(\frac{k^{2}}{\mu^{2}}\right)^{\gamma^{*} / 2} .
$$

Treating the running coupling as a constant is a convenience approximation to consider the effect of anomalous dimension.

We now return to the pair condensate $\left\langle\bar{\psi}_{i} \psi^{i}\right\rangle$. From the eq. (3.65), we get

$$
\left\langle\bar{\psi}_{R} \psi_{L}\right\rangle \frac{1-\gamma_{5}}{2}=\lim _{p \rightarrow \infty} \frac{\int d^{4} x e^{i p x}\left\langle T \psi_{L}^{i}(x) \bar{\psi}_{R}^{i}(0)\right\rangle}{U_{\bar{\psi} \psi}^{i i}(p, g, \mu)} .
$$


By substituting the result of eq.(3.78), we obtain

$$
\langle\bar{\psi} \psi\rangle=-\lim _{p \rightarrow \infty} \frac{4 N_{c}}{3 c g^{* 2} C_{2}}\left(\frac{\mu^{2}}{p^{2}}\right)^{\gamma^{*} / 2} p^{2} \Sigma\left(-p^{2}\right)
$$

where we use the solution of Schwinger-Dyson equation in Landau gauge $\left(A\left(k^{2}\right)=1\right.$ and $B\left(k^{2}\right)=\Sigma\left(-k^{2}\right)$ in eq.(3.41) $)$. If $\Sigma=0$, the pair condensate vanishes. But if $\Sigma$ is non-trivial and has the asymptotic behavior

$$
\lim _{p \rightarrow \infty} \Sigma\left(-p^{2}\right)=\text { constant } \times \frac{1}{p^{2}}\left(\frac{p^{2}}{\mu^{2}}\right)^{\gamma^{*} / 2},
$$

then the condensate takes finite value.

We have tried to calculate the value of the pair condensate in QCD. Since the mass function $\Sigma$ and the anomalous dimension can not perfectly be estimated, we can not proof the existence of the pair condensate in QCD. If we believe the existence of the pair condensate, the anomalous dimension can be obtained form eq.(3.81) and eq.(3.63). Since

eq. (3.63) suggests that the asymptotic behavior of mass function is $1 / \sqrt{p^{2}}$ (up to log), we know that the anomalous dimension must be unity, $\gamma^{*}=1$, for the finite condensate. This value of $\gamma^{*}$ is large compared with the naive expectation from the perturbative QCD.

The Schwinger-Dyson equation which is considered in this section is called QED-like, since the non-abelian effect of gauge interaction (non-abelian vertex) is not included. The form of the equation is just the same in QED, except for the Casimir coefficient $C_{2}$. The effect can be approximately included by replacing the coupling constant by running coupling (Higashijima approximation [20]). The approximation results the small anomalous dimension $\gamma^{*} \sim 0$. We can expect that the slower running coupling means the larger anomalous dimension $\gamma^{*}$.

\subsection{Decay constant of Nambu-Goldstone bosons}

The Nambu-Goldstone theorem says that the Nambu-Goldstone bosons couple with the broken currents as

$$
\left\langle 0\left|J_{\mu}^{a}(0)\right|(N G)^{b}(q)\right\rangle=i q_{\mu} I^{a b}\left(q^{2}\right) .
$$

The value $I^{a b}\left(q^{2}=0\right)=f^{a b}$ is generally referred as the decay constant, because the pions as the pseudo-Nambu-Goldstone bosons couple with the weak currents and decay to the leptons.

In the following we consider QCD in which the chiral symmetry is spontaneously broken by the quark condensate. The currents which couple with the Nambu-Goldstone bosons are the axial currents $\bar{q} \gamma_{\mu} \gamma_{5} T^{a} q$. The above amplitude can be written as (fig.(3.3))

$$
-\operatorname{tr} \int \frac{d^{4} k}{(4 \pi)^{4} i} S(k) \gamma_{\mu} \gamma_{5} T^{a} S(q+k) P^{b}(q+k, k)=i q_{\mu} I^{a b}\left(q^{2}\right),
$$

where $S$ is the full quark propagator, and $P^{b}(q+k, k)$ is the Bethe-Salpeter amplitude. 
Figure 3.3: Coupling between the Nambu-Goldstone bosons and currents.

Figure 3.4: Schwinger-Dyson equation for the axial vertex.

Bethe-Salpeter amplitude describes the coupling between the quarks and the NambuGoldstone boson. By differentiating both side with respect to $q^{\nu}$ and taking limit $q \rightarrow 0$, we obtain

$$
\begin{aligned}
i g_{\mu \nu} f^{a b}= & -\operatorname{tr} \int \frac{d^{4} k}{(4 \pi)^{4} i} S(k) \gamma_{\mu} \gamma_{5} T^{a}\left(\frac{\partial}{\partial q^{\nu}} S(q+k)\right)_{q \rightarrow 0} P^{b}(k, k) \\
& -\operatorname{tr} \int \frac{d^{4} k}{(4 \pi)^{4} i} S(k) \gamma_{\mu} \gamma_{5} T^{a} S(k)\left(\frac{\partial}{\partial q^{\nu}} P^{b}(q+k, k)\right)_{q \rightarrow 0} .
\end{aligned}
$$

To discuss the ladder approximation of this formula, we consider the Schwinger-Dyson equation for the axial vertex (fig.(3.4))

$$
\Gamma_{5 \mu}^{a}(p, p+q)=\gamma_{\mu} \gamma_{5} T^{a}-\operatorname{tr} \int \frac{d^{4} k}{(4 \pi)^{4} i} \Gamma_{5 \mu}^{a}(k, k+q) S(k+q) K(k+q, p, p+q) S(k),
$$

where $K$ is the set of the horizontally one-particle irreducible diagrams. The axial vertex can be decomposed in two parts as (fig.(3.5))

$$
\Gamma_{5 \mu}^{a}(p, p+q)=i q_{\mu} I^{a b}\left(q^{2}\right) \frac{1}{-q^{2}} P^{b}(p, p+q)+\tilde{\Gamma}_{5 \mu}^{a}(p, p+q) .
$$

The first term is the $1 / q^{2}$ pole contribution of Nambu-Goldstone boson, and the second term is the another contribution which is regular at $q=0$. The functions $\tilde{\Gamma}_{5 \mu}^{a}(p, p+q)$, $P^{a}(p, p+q)$, and $K(k+q, p, p+q)$ are regular at $q=0$. By substituting this to the Schwinger-Dyson equation, we get

$$
\begin{aligned}
& i q_{\mu} I^{a b}\left(q^{2}\right) \frac{1}{-q^{2}}\left\{P^{b}(p, p+q)+\operatorname{tr} \int \frac{d^{4} k}{(4 \pi)^{4} i} P^{b}(k, k+q) S(k+q) K(k+q, p, p+q) S(k)\right\} \\
& =\gamma_{\mu} \gamma_{5} T^{a}-\tilde{\Gamma}_{5 \mu}^{a}(p, p+q)-\operatorname{tr} \int \frac{d^{4} k}{(4 \pi)^{4} i} \tilde{\Gamma}_{5 \mu}^{a}(k, k+q) S(k+q) K(k+q, p, p+q) S(k) .
\end{aligned}
$$

It is expected that each of the functions $P^{a}(p, p+q)$ and $\tilde{\Gamma}_{5 \mu}^{a}(p, p+q)$ satisfies the independent equation in the limit $q \rightarrow 0$, since they appear as the different order of $q$ in eq.(3.86). Therefore, we get in $q \rightarrow 0$ limit

$$
\begin{gathered}
i q_{\mu} I^{a b}\left(q^{2}\right) \frac{1}{-q^{2}}\left\{P^{b}(p, p+q)+\operatorname{tr} \int \frac{d^{4} k}{(4 \pi)^{4} i} P^{b}(k, k+q) S(k+q) K(k+q, p, p+q) S(k)\right\}=O(q), \\
\gamma_{\mu} \gamma_{5} T^{a}-\tilde{\Gamma}_{5 \mu}^{a}(p, p+q)-\operatorname{tr} \int \frac{d^{4} k}{(4 \pi)^{4} i} \tilde{\Gamma}_{5 \mu}^{a}(k, k+q) S(k+q) K(k+q, p, p+q) S(k)=O(q) .
\end{gathered}
$$


Figure 3.5: Decomposition of the axial vertex.

Figure 3.6: Graphic representation of eq.(3.90). Slash denotes the derivative with respect to $q$.

Then, we obtain

$$
\begin{aligned}
& P^{b}(p, p+q)=-\operatorname{tr} \int \frac{d^{4} k}{(4 \pi)^{4} i} P^{b}(k, k+q) S(k+q) K(k+q, p, p+q) S(k)+O\left(q^{2}\right), \\
& \tilde{\Gamma}_{5 \mu}^{a}(p, p+q)=\gamma_{\mu} \gamma_{5} T^{a}-\operatorname{tr} \int \frac{d^{4} k}{(4 \pi)^{4} i} \tilde{\Gamma}_{5 \mu}^{a}(k, k+q) S(k+q) K(k+q, p, p+q) S(k)+O(q) .
\end{aligned}
$$

The first equation is called Bethe-Salpeter equation for the Nambu-Goldstone boson vertex.

The vertex $\gamma_{\mu} \gamma_{5} T^{a}$ can be eliminated from eq.(3.84) by using eq.(3.91).

$$
\begin{aligned}
& i g_{\mu \nu} f^{a b}=-\operatorname{tr} \int_{k} S(k) \tilde{\Gamma}_{5 \mu}^{a}(k, k)\left(\frac{\partial}{\partial q^{\nu}} S(q+k)\right)_{q \rightarrow 0} P^{b}(k, k) \\
& -\operatorname{tr} \int_{k, k^{\prime}} S(k) \operatorname{tr}\left\{\tilde{\Gamma}_{5 \mu}^{a}\left(k^{\prime}, k^{\prime}\right) S\left(k^{\prime}\right) K\left(k^{\prime}, k, k\right) S\left(k^{\prime}\right)\right\}\left(\frac{\partial}{\partial q^{\nu}} S(q+k)\right)_{q \rightarrow 0} P^{b}(k, k) \\
& \quad-\operatorname{tr} \int_{k} S(k) \tilde{\Gamma}_{5 \mu}^{a}(k, k) S(k)\left(\frac{\partial}{\partial q^{\nu}} P^{b}(q+k, k)\right)_{q \rightarrow 0} \\
& \quad-\operatorname{tr} \int_{k, k^{\prime}} S(k) \operatorname{tr}\left\{\tilde{\Gamma}_{5 \mu}^{a}\left(k^{\prime}, k^{\prime}\right) S\left(k^{\prime}\right) K\left(k^{\prime}, k, k\right) S\left(k^{\prime}\right)\right\} S(k)\left(\frac{\partial}{\partial q^{\nu}} P^{b}(q+k, k)\right)_{q \rightarrow 0},
\end{aligned}
$$

where $\int_{k}$ means $\int d^{4} k /(4 \pi)^{4} i$. Moreover, the derivative of $P^{b}$ can be eliminated by using eq.(3.90) (fig.(3.6)).

$$
\begin{aligned}
& i g_{\mu \nu} f^{a b}=-\operatorname{tr} \int_{k} S(k) \tilde{\Gamma}_{5 \mu}^{a}(k, k)\left(\frac{\partial}{\partial q^{\nu}} S(q+k)\right)_{q \rightarrow 0} P^{b}(k, k) \\
& \quad+\operatorname{tr} \int_{k, k^{\prime}} S(k) \operatorname{tr}\left\{\tilde{\Gamma}_{5 \mu}^{a}\left(k^{\prime}, k^{\prime}\right) S\left(k^{\prime}\right)\left(\frac{\partial}{\partial q^{\nu}} K\left(q+k^{\prime}, p, p+q\right)\right)_{q \rightarrow 0} S\left(k^{\prime}\right)\right\} S(k) P^{b}(k, k) .
\end{aligned}
$$

Now we consider the ladder approximation of this formula. In ladder approximation with Landau gauge, the renormalization of the axial vertex is trivial. We can show that by using the chiral Ward-Takahashi identity $\mathrm{f}$

$$
k^{\mu} \Gamma_{5 \mu}^{a}(p, p+k)=S^{-1}(p) \gamma_{5} T^{a}+\gamma_{5} T^{a} S^{-1}(p+k)
$$

\footnotetext{
${ }^{4}$ Here, we neglect the contribution of the chiral anomaly.
} 
and the fact that the renormalization of the quark field is trivial $\left(Z_{2}=1\right)$ as we have already shown in previous section. Therefore, we should set $K=0$ in eq.(3.91) and get $\tilde{\Gamma}_{5 \mu}^{a}(k, k)=\gamma_{\mu} \gamma_{5} T^{a}$. The eq.(3.93) becomes

$$
i g_{\mu \nu} f^{a b}=-\operatorname{tr} \int \frac{d^{4} k}{(4 \pi)^{4} i} S(k) \gamma_{\mu} \gamma_{5} T^{a}\left(\frac{\partial}{\partial q^{\nu}} S(q+k)\right)_{q \rightarrow 0} P^{b}(k, k) .
$$

This equation also can be get by simply neglecting the second term in eq.(3.84). Neglecting the derivative of Bethe-Salpeter amplitude corresponds to neglecting the effect of the axial vector resonance in it .

The Bethe-Salpeter amplitude at zero momentum transfer, $P(k, k)$, can be expressed by the mass function $\Sigma(k)$. By taking the limit $k \rightarrow 0$ in eq.(3.94), we extract the contribution of Nambu-Goldstone boson pole in the axial vertex function, and obtain

$$
-i f^{a b} P^{b}(k, k)=S^{-1}(k) \gamma_{5} T^{a}+\gamma_{5} T^{a} S^{-1}(k) .
$$

Since we are taking ladder approximation with Landau gauge,

$$
f^{a b} P^{b}(k, k)=2 i \Sigma\left(-k^{2}\right) \gamma_{5} T^{a} .
$$

By substituting this equation into eq.(3.95), we get

$$
\left(f f^{T}\right)^{a b}=\frac{N_{c}}{4 \pi^{2}} \delta^{a b} \int d k^{2} \frac{k^{2} \Sigma\left(k^{2}\right)}{\left(\Sigma^{2}\left(k^{2}\right)+k^{2}\right)^{2}}\left\{\Sigma\left(k^{2}\right)-\frac{1}{2} k^{2} \frac{d \Sigma\left(k^{2}\right)}{d k^{2}}\right\} .
$$

This equation is expressed in the Euclidean momentum space. If the $S U\left(N_{f}\right)_{V}$ symmetry is not broken, the decay constant matrix becomes diagonal. In that case, we have

$$
f^{2}=\frac{N_{c}}{4 \pi^{2}} \int d k^{2} \frac{k^{2} \Sigma\left(k^{2}\right)}{\left(\Sigma^{2}\left(k^{2}\right)+k^{2}\right)^{2}}\left\{\Sigma\left(k^{2}\right)-\frac{1}{2} k^{2} \frac{d \Sigma\left(k^{2}\right)}{d k^{2}}\right\} .
$$

This formula is called Pagels-Stokar formula 16. We can obtain the value of the decay constant, if the ladder Schwinger-Dyson equation for quark propagator is solved.

\subsection{Cornwall-Jackiw-Tomboulis effective action}

In this section we introduce the Cornwall-Jackiw-Tomboulis (CJT) effective action 17. The Schwinger-Dyson equation for quark propagator is systematically derived from this effective action. It is also useful when we consider the more complicated system like that we will estimate in chapter 6 and 7 .

The generating functional for the Green functions in QCD is

$$
\begin{aligned}
& Z[K, J, \bar{J}]=\frac{1}{Z} \int \mathcal{D} \psi \mathcal{D} \bar{\psi} \mathcal{D} G \exp i \int d^{4} x\{\bar{\psi}(x)(i \not \partial-m+g G(x)) \psi(x) \\
& \left.-\frac{1}{2} \operatorname{tr}\left(F^{\mu \nu}(x) F_{\mu \nu}(x)\right)+\int d^{4} y \bar{\psi}(x) K(x, y) \psi(y)+\bar{J}(x) \psi(x)+\bar{\psi}(x) J(x)\right\}
\end{aligned}
$$

\footnotetext{
5 The mixing between the axial vector resonance and Nambu-Goldstone boson is proportional to $q$
} 
where $J(x)$ and $\bar{J}(x)$ are the local sources, and $K(x, y)$ is the non-local source. The normalization $Z[K=0, J=0, \bar{J}=0]=1$ is understood. The connected Green functions like $\langle T \psi \psi \cdots \bar{\psi} \bar{\psi} \cdots\rangle_{c}$ are obtained by differentiating $Z[K, J, \bar{J}]$ with respect to $J$ 's and $\bar{J}$ 's. The full quark propagator is obtained by differentiating it with respect to $-i K$. We integrate out the quark and gluon fields and define the CJT effective action.

Using the derivative with respect to the sources gives

$$
\begin{gathered}
Z[K, J, \bar{J}]=\frac{1}{Z} \exp i \int\left\{-\frac{\delta}{i \delta J}(i \not \partial-m) \frac{\delta}{i \delta \bar{J}}-\frac{\delta}{i \delta J} K \frac{\delta}{i \delta \bar{J}}\right\} \\
\times \int \mathcal{D} \psi \mathcal{D} \bar{\psi} \mathcal{D} G \exp i \int\left\{\bar{\psi} g \not G \psi-\frac{1}{2} \operatorname{tr}\left(F^{\mu \nu}(x) F_{\mu \nu}(x)\right)\right. \\
+\bar{J}(x) \psi(x)+\bar{\psi}(x) J(x)\} .
\end{gathered}
$$

We introduce the full quark propagator $S$.

$$
\begin{aligned}
& Z[K, J, \bar{J}]=\frac{1}{Z} \exp i \int\{\operatorname{tr} {\left.\left[\left(i \not \partial-m-i S^{-1}\right) \frac{\delta}{i \delta \bar{J}} \frac{\delta}{i \delta J}\right]+\operatorname{tr}\left[K \frac{\delta}{i \delta \bar{J}} \frac{\delta}{i \delta J}\right]\right\} } \\
& \times \int \mathcal{D} \psi \mathcal{D} \bar{\psi} \mathcal{D} G \exp i \int\left\{\bar{\psi} i S^{-1} \psi+\bar{\psi} g \operatorname{G} \psi-\frac{1}{2} \operatorname{tr}\left(F^{\mu \nu}(x) F_{\mu \nu}(x)\right)\right. \\
&+\bar{J}(x) \psi(x)+\bar{\psi}(x) J(x)\}
\end{aligned}
$$

At this stage, $S$ need not be the full propagator, but we will ensure it later. The integration out on the gluon field gives the set of connected two-particle irreducible (2PI) vacuum diagrams $D[S, J, \bar{J}]$.

$$
\begin{gathered}
Z[K, J, \bar{J}]=\frac{1}{Z} \exp i \int\left\{\operatorname{tr}\left[\left(i \not \partial-m-i S^{-1}\right) \frac{\delta}{i \delta \bar{J}} \frac{\delta}{i \delta J}\right]+\operatorname{tr}\left[K \frac{\delta}{i \delta \bar{J}} \frac{\delta}{i \delta J}\right]\right\} \\
\times \int \mathcal{D} \psi \mathcal{D} \bar{\psi} \exp i \int\left\{\bar{\psi} i S^{-1} \psi+\bar{J}(x) \psi(x)+\bar{\psi}(x) J(x)\right\} \times e^{D[S, J, \bar{J}]} .
\end{gathered}
$$

The fact that $S$ is the full propagator makes the set of the vacuum diagrams to be the two-particle irreducible one. Now we can integrate out the quark fields.

$$
\begin{aligned}
Z[K, J, \bar{J}]=\frac{1}{Z} \exp i \int\{\operatorname{tr}[(i \not \partial- & \left.\left.\left.-i S^{-1}\right) \frac{\delta}{i \delta \bar{J}} \frac{\delta}{i \delta J}\right]+\operatorname{tr}\left[K \frac{\delta}{i \delta \bar{J}} \frac{\delta}{i \delta J}\right]\right\} \\
& \times \operatorname{Det}\left(-S^{-1}\right) e^{-\int \bar{J} S J} e^{D[S, J, \bar{J}]}
\end{aligned}
$$

Carrying out the functional derivatives and setting $J$ and $\bar{J}$ to zero gives

$$
Z[K]=\frac{1}{Z} \exp \left\{\ln \operatorname{Det}\left(-S^{-1}\right)-i \operatorname{Tr}\left[\left(i \not \partial-m-i S^{-1}\right) S\right]-i \operatorname{Tr}[K S]+D[S]\right\},
$$

where $\operatorname{Tr}$ denotes the trace in the functional space in addition to the trace in the spinor and flavor spaces. The generating functional for the connected Green functions, $W[K]=$ $\ln Z[K]$, is obtained as

$$
W[K]=\ln \operatorname{Det}\left(-S^{-1}\right)-i \operatorname{Tr}\left[\left(i \not \partial-m-i S^{-1}\right) S\right]-i \operatorname{Tr}[K S]+D[S],
$$


Figure 3.7: The vacuum diagram for ladder approximation.

where we neglect a constant $\ln Z$. Now we can say that $S$ is really the full propagator, since

$$
-\left.\frac{1}{i} \frac{\delta W[K]}{\delta K}\right|_{K \rightarrow 0}=S .
$$

The Legendre transformation gives the CJT effective action as

$$
\begin{aligned}
\Gamma[S] & =W[K]+i \operatorname{Tr}[K S] \\
& =\ln \operatorname{Det}\left(-S^{-1}\right)-i \operatorname{Tr}\left[\left(i \not \partial-m-i S^{-1}\right) S\right]+D[S]
\end{aligned}
$$

with

$$
\frac{\delta \Gamma[S]}{\delta S}=i K
$$

In momentum space it becomes

$$
\Gamma[S]=\ln \operatorname{Det}^{-1}(k)-\operatorname{Tr}[(\not k-m) S(k)]+D[S] .
$$

The difference on the constant and over all factor $(2 \pi)^{4} \delta^{4}(0)$ (four dimensional volume) is neglected.

Since $K=0$ in the real system, the condition eq. (3.109) becomes the stationary condition of the effective action. The condition is equivalent to the Schwinger-Dyson equation for propagator. If we take the $2 \mathrm{PI}$ vacuum diagram in fig.(3.7), namely

$$
D[S]=-\frac{1}{2} \operatorname{tr} \int \frac{d^{4} p}{(2 \pi)^{4} i} \frac{d^{4} q}{(2 \pi)^{4} i} g \gamma^{\mu} T^{a} S(p) g \gamma^{\nu} T^{a} S(q) D_{\mu \nu}^{\text {free }}(p-q),
$$

the condition gives the Schwinger-Dyson equation in ladder approximation (cf. eq.(3.39))

$$
S^{-1}(k)=-(\not k-m)-\int \frac{d^{4} q}{(2 \pi)^{4} i} g \gamma^{\mu} T^{a} S(q) g \gamma^{\nu} T^{a} D_{\mu \nu}^{\text {free }}(k-q) .
$$

The effective action formalism is convenient for the systematic analysis. We will use this formalism in chapter 6 and 7 to estimate the dynamics of the tumbling gauge theory. 


\section{Chapter 4}

\section{Technicolor model}

The standard model which describes the three fundamental interactions (electromagnetic, weak, and strong interactions) is consistent with almost all the experiments. Especially, the agreement with the recent precision experiments in LEP is excellent. It is believed that we finally get the true theory of electromagnetic and weak interactions.

The theory of the electromagnetic and weak interactions is based on the gauge principle. The gauge symmetry of these interactions is the electroweak symmetry $S U(2)_{L} \times$ $U(1)_{Y}$ which describes the four massless gauge bosons. The gauge symmetry is spontaneously broken to the electromagnetic gauge symmetry $U(1)_{e m}$ by the vacuum expectation value of the Higgs field. The three of four gauge bosons become massive by the Higgs mechanism, and they mediate the weak interaction. The mass explains the fact that the weak interaction is the short range one. Residual one gauge boson remains massless, and it mediates the electromagnetic interaction.

It is interesting that the electroweak symmetry forbids the mass of the quarks and leptons, since it is the chiral symmetry. Electroweak symmetry must be broken also to generate the mass of the quarks and leptons. In the standard model, they get their masses through the Yukawa interactions like

$$
\mathcal{L}_{\text {Yukawa }}=-g_{\psi} \bar{\psi}_{L i} \psi_{R} \Phi^{i}+\text { h.c. },
$$

where $\psi$ is the quark or lepton, $\Phi$ is the Higgs field, and $i$ is the index of the doublet of the

$S U(2)_{L}$ symmetry. The vacuum expectation value of the Higgs field, $\langle\Phi\rangle=\left(\begin{array}{ll}0 & v / \sqrt{2}\end{array}\right)^{T}$, which breaks the electroweak symmetry gives the mass to the fermions as $m_{\psi}=g_{\psi} v / \sqrt{2}$. The vacuum expectation value of the Higgs field is not only the origin of the mass of the weak bosons, but also the origin of the mass of the quarks and leptons.

The standard model, however, has some theoretical problems in it. In the next section, we will explain it as the motivation for technicolor theory in which the vacuum expectation value of the Higgs field is replaced by the fermion pair condensate $\langle\bar{T} T\rangle \neq 0$. In section 2 , the one family model of the technicolor theory is explained as an example. 


\subsection{Motivation for technicolor}

One of the problems of the standard model is the fact that the masses of the quarks and leptons are not predicted. The angles of the quark mixing and $\mathrm{CP}$ violating phase are not also predicted, since the origin is the same of the masses [3]. There is no principle to predict the Yukawa couplings which are given as the free parameters. We still do not know the origin of mass. The existence of certain new physics should be expected at the high energy beyond the standard model.

There is also the problem which is called fine tuning problem, or naturalness problem, or hierarchy problem 21]. The parameters in the Higgs potential must be fine tuned, if the standard model is looked upon as an effective theory. Suppose that the standard model is valid until certain high energy scale $\Lambda$ (GUT scale, for example.). Then the Higgs potential

$$
V(\Phi)=-m^{2} \Phi^{\dagger} \Phi+\frac{\lambda}{4}\left(\Phi^{\dagger} \Phi\right)^{2}
$$

receive the radiative correction as

$$
V(\Phi)_{\text {one-loop }}=-\left(m^{2}-\lambda \frac{\Lambda^{2}}{16 \pi^{2}}\right) \Phi^{\dagger} \Phi+\frac{\lambda}{4}\left(\Phi^{\dagger} \Phi\right)^{2}
$$

up to the log correction. The vacuum expectation value of the Higgs field $\Phi$ is

$$
\langle\Phi\rangle=\frac{v}{\sqrt{2}}\left(\begin{array}{l}
0 \\
1
\end{array}\right)=\sqrt{\frac{2\left(m^{2}-\lambda \Lambda^{2} / 16 \pi\right)}{\lambda}}\left(\begin{array}{l}
0 \\
1
\end{array}\right) .
$$

This value must be the electroweak scale $v \simeq 250 \mathrm{GeV}$ to give the realistic weak boson mass. We must take the mass $m^{2}$ a large value, and fine tune to cancel the large value $\lambda \Lambda^{2} / 16 \pi$ (like $1.00000000000001-1=0.00000000000001$ ) . This work must be iterated order by order in loop calculation. This unnatural situation means that we still do not know the mechanism of the electroweak symmetry breaking (Why the scale is so small?). The "natural" physics beyond the standard model should exist.

Technicolor theory [7] is one of the candidate of the new physics. It is a "natural" theory, and has a possibility to explain the mass generation. The Higgs field as an elementary particle is eliminated and the new fermions (technifermions) are introduced with the additional strong interaction (technicolor interaction). The technifermions are not the singlet of the electroweak symmetry $S U(2)_{L} \times U(1)_{Y}$ so that the condensate $\langle\bar{T} T\rangle \neq 0$ due to the technicolor interaction spontaneously breaks the symmetry. The vacuum expectation value of the elementary Higgs field is replaced by the fermion pair condensate $\langle\bar{T} T\rangle$. Namely, the electroweak symmetry is dynamically broken \&. The Nambu-Goldstone bosons which are eaten by the weak bosons are the composite particles of the technifermions. The physical Higgs particle is also the composite one. Its width will be very broad, since it strongly decays to the pair of the Nambu-Goldstone bosons.

Consider the hierarchy of the scales between the weak scale and the scale of certain new physics. If we renormalize the gauge coupling constants at the renormalization point

${ }^{1}$ This manipulation is the same of the renormalization. But here, $\Lambda$ is the physical ultraviolet cut off.

2 This is very similar to the chiral symmetry breaking in QCD due to the quark condensate. 
$\mu=M$, then we have the running gauge couplings at one-loop level as

$$
\begin{aligned}
\alpha_{T C}^{-1}(\mu) & =\alpha_{T C}^{-1}(M)+c_{T C} \ln \left(\frac{\mu}{M}\right), \\
\alpha_{\text {new }}^{-1}(\mu) & =\alpha_{\text {new }}^{-1}(M)+c_{\text {new }} \ln \left(\frac{\mu}{M}\right),
\end{aligned}
$$

where $\alpha_{T C}$ is the gauge coupling of technicolor interaction, $\alpha_{\text {new }}$ is the gauge coupling constant by which the scale of new physics is specified. Both coefficients $c_{T C}$ and $c_{\text {new }}$ take positive values so that the gauge interactions are the asymptotically free ones, and these magnitude is supposed to be of the order unity. The technicolor condensate scale $M_{\text {weak }}$ (weak scale) and the new physics scale $M_{\text {new }}$ are roughly defined by

$$
\begin{array}{rll}
M_{\text {weak }}: & \alpha_{T C}\left(M_{\text {weak }}\right)=1, \\
M_{\text {new }}: & \alpha_{T C}\left(M_{\text {new }}\right)=1 .
\end{array}
$$

These scales are defined as the scales at which the non-perturbative effects of the gauge interactions become important. The hierarchy $M_{\text {new }} \gg M_{\text {weak }}$ can be obtained by choosing $\alpha_{T C}(M)$ without the fine tuning. For example, consider the case $M_{\text {new }} / M_{\text {weak }} \simeq 10^{13}$. Then, we can choose $\alpha_{T C}(M)$ as

$$
\frac{1-\alpha_{\text {new }}^{-1}(M)}{c_{\text {new }}}-\frac{1-\alpha_{T C}^{-1}(M)}{c_{T C}}=\ln \frac{M_{\text {new }}}{M_{\text {weak }}} \simeq 32
$$

without the fine tuning. The point is that the large difference of the scales only comes through the form of logarithm.

To generate the masses of the quarks and leptons, we must introduce new interactions which act the role of the Yukawa couplings in the Standard model. The technifermion condensate breaks the chiral symmetry in the technifermion sector, but it does not break the one in the ordinary fermion sector. These two chiral symmetry should be connected to generate the ordinary fermion mass through the technifermion condensation. The new interactions should reduce these two chiral symmetry to a single one. The typical form of the new interaction is

$$
\mathcal{L}_{\text {new }}=\frac{g_{L}^{\psi} g_{R}^{\psi}}{M_{\psi}^{2}}\left(\bar{T}_{L i} T_{R}\right)\left(\bar{\psi}_{R} \psi_{L}^{i}\right)+\text { h.c. },
$$

where $\psi$ denotes the ordinary fermion, and $i$ is the index of the $S U(2)_{L}$ doublet. $g_{L}^{\psi}$ and $g_{R}^{\psi}$ are the coupling constants and $M_{\psi}$ is the appropriate energy scale. This interaction is not invariant under the independent chiral rotation of the ordinary fermions or the technifermions, but it is invariant under the simultaneous chiral rotation of both fermions (Of course, it must be invariant under the electroweak gauge transformation). The ordinary fermions get their masses

$$
m_{\psi} \simeq \frac{g_{L}^{\psi} g_{R}^{\psi}}{M_{\psi}^{2}}\left|\left\langle\bar{T}_{L} T_{R}\right\rangle\right|
$$

through the technifermion condensate $\langle\bar{T} T\rangle \neq 0$ ? The variety of the masses, the quark mixing angles, and the $\mathrm{CP}$ violation in the weak interaction originate from the structure

\footnotetext{
${ }^{3}$ The mean field approximation is used. We will treat it more rigorously in next chapter.
} 
of the coupling $g_{L}^{\psi} g_{R}^{\psi} / M_{\psi}^{2}$. As the origin of these interaction, there is an interesting idea so called extended technicolor theory [6].

In extended technicolor theory, the technicolor gauge interaction is extended to generate the above interactions as the effective four fermion interactions. The extended gauge multiplets contain both ordinary fermions and technifermions.

$\overbrace{\left(\begin{array}{lllll}\psi_{1} & \psi_{2} & \psi_{3} & \underbrace{T_{1}}_{\text {Technicolor }} T_{2} & T_{3}\end{array}\right)}^{\text {Extended technicolor }}$

When the extended gauge group is spontaneously broken to the technicolor gauge group by the effect of certain dynamics, the gauge bosons which cause the transition of the ordinary fermions to the technifermions (so called sideways gauge bosons) become massive. Such gauge bosons generate the effective four fermion interaction like

$$
\mathcal{L}_{4 F}=\left(\bar{T}_{L} \frac{g_{L}^{\psi}}{\sqrt{2}} \gamma_{\mu} \psi_{L}\right) \frac{1}{-M_{\psi}^{2}}\left(\bar{\psi}_{R} \frac{g_{R}^{\psi}}{\sqrt{2}} \gamma_{\mu} T_{R}\right)+\text { h.c... }
$$

Firtz transformation gives the four fermion interaction as eq.(4.10).

The mass difference between the ordinary fermions is due to the difference of the scales $M_{\psi}$ and the couplings $g_{L}^{\psi}$ and $g_{R}^{\psi}$. It is naturally expected that the mass difference between the different generations comes from the difference of the scales $M_{\psi}$, and the mass splitting in each generation comes from the difference of the couplings $g_{L}^{\psi}$ and $g_{R}^{\psi}$. The mass splitting in a weak doublet comes from the difference of $g_{R}^{\psi}$, since $g_{L}^{\psi}$ must be common in a weak doublet according to the gauge invariance. For example, $g_{R}^{\psi}$ for u-quark should be different from the one for d-quark: $g_{R}^{u} \neq g_{R}^{d}$. Therefore, the extended gauge theory should be the chiral gauge theory with many (at least three) mass scales.

No concrete realistic model of the extended technicolor theory has been proposed until now, since the dynamics of the gauge theory, especially for the dynamics of the chiral gauge theory, is not completely understood. The dynamics of the tumbling gauge theory is expected as the dynamics of the extended technicolor gauge interaction. The theory is a class of the chiral gauge theory in which many mass scales are expected. It will be discussed in detail in chapter 6 .

In next section we introduce a model so called one-family model [22]. While the model is not realistic, it is worth studying to get the insight about what phenomenology generally appears in the technicolor theory.

\subsection{One-family model}

Consider the technicolor gauge group $S U\left(N_{T C}\right)$, and introduce the one family technifermions (with right-handed techni-neutrino)

$$
\begin{aligned}
\left(\begin{array}{c}
U \\
D
\end{array}\right)_{L} & \sim(2,1 / 6) \\
U_{R} & \sim(1,2 / 3),
\end{aligned}
$$




$$
\begin{aligned}
D_{R} & \sim(1,-1 / 3), \\
\left(\begin{array}{c}
N \\
E
\end{array}\right)_{L} & \sim(2,-1 / 2), \\
N_{R} & \sim(1,0), \\
E_{R} & \sim(1,-1),
\end{aligned}
$$

as the fundamental representation of the gauge group. Here, $(2,1 / 6)$ means the $S U(2)_{L}$ doublet with hypercharge $1 / 6$, and so on. The one-family technifermion is considered to cancel the gauge anomalies $t$. The technifermions condensate is expected as

$$
\langle\bar{U} U\rangle=\langle\bar{D} D\rangle=\langle\bar{N} N\rangle=\langle\bar{E} E\rangle \neq 0
$$

like the quark condensate in QCD. There is the (approximate) chiral symmetry $S U(8)_{L} \times$ $S U(8)_{R}$ in the technifermion sector, and it is spontaneously broken to $S U(8)_{V}$ by the condensates. Therefore, 64 Nambu-Goldstone bosons should exist. The contents are

$$
\begin{aligned}
\theta^{a i} & \sim \bar{Q} i \gamma_{5} \lambda^{a} \tau^{i} Q, \\
\theta^{a} & \sim \bar{Q} i \gamma_{5} \lambda^{a} Q, \\
T^{\alpha i} & \sim \bar{Q}^{\alpha} i \gamma_{5} \tau^{i} L, \quad\left(\bar{T}^{\alpha i} \sim \bar{L}^{\alpha} i \gamma_{5} \tau^{i} Q\right), \\
T^{\alpha} & \sim \bar{Q}^{\alpha} i \gamma_{5} L, \quad\left(\bar{T}^{\alpha} \sim \bar{L}^{\alpha} i \gamma_{5} Q\right), \\
\Pi^{i} & \sim \bar{Q} i \gamma_{5} \tau^{i} Q+\bar{L} i \gamma_{5} \tau^{i} L, \\
P^{i} & \sim \bar{Q} i \gamma_{5} \tau^{i} Q-3 \bar{L} i \gamma_{5} \tau^{i} L, \\
P^{0} & \sim \bar{Q} i \gamma_{5} Q-3 \bar{L} i \gamma_{5} L,
\end{aligned}
$$

where $Q=\left(\begin{array}{ll}U & D\end{array}\right)^{T}$ and $L=\left(\begin{array}{ll}N & E\end{array}\right)^{T}$. There are 56 colored pseudoscalars: $\theta^{a i}$ and $\theta^{a}$ are the color octets (index $a$ ), $T^{\alpha i}$ and $T^{\alpha}$ are the color triplets (index $\alpha$ ) which are called leptoquarks. $\Pi^{i}$ are identified as the would be Nambu-Goldstone bosons which are eaten by the weak gauge bosons. There are four other color singlet Nambu-Goldstone bosons: $P^{i}$ and $P^{0} . P^{3}$ and $P^{0}$ is called axions which are the singlets of both QCD and QED.

The current of the broken chiral symmetry is

$$
J_{\mu}^{a}=\left(\begin{array}{cc}
\bar{Q} & \bar{N}
\end{array}\right) \gamma_{\mu} \gamma_{5} T^{a}\left(\begin{array}{c}
Q \\
N
\end{array}\right),
$$

where $T^{a}$ is the generators of $S U(8)$, and the decay constant of the Nambu-Goldstone bosons $F_{\pi}$ is defined as

$$
\left\langle 0\left|J_{\mu}^{a}\right| N G^{b}(k)\right\rangle=i k_{\mu} \delta^{a b} F_{\pi} .
$$

The current contains the weak axial current $j_{\mu}^{i}$ as

$$
j_{\mu}^{i}=\sqrt{N_{D}} J_{\mu}^{a=i},
$$

\footnotetext{
${ }^{4}$ In view of the gauge anomaly cancellation, the one-doublet model in which the one weak doublet technifermion is introduced is more simple. But since the technifermions have the unusual electric charge $Q= \pm 1 / 2$ in that case, it is not cosmologically acceptable.
} 
where $N_{D}=4$ is the number of the weak doublet and

$$
T^{a=i}=\frac{1}{\sqrt{N_{D}}}\left(\begin{array}{cccc}
\frac{\tau^{i}}{2} & & & \\
& \frac{\tau^{i}}{2} & & \\
& & \frac{\tau^{i}}{2} & \\
& & & \frac{\tau^{i}}{2}
\end{array}\right)
$$

since the normalization $\operatorname{tr}\left(T^{a} T^{b}\right)=\delta^{a b} / 2$. Therefore, the mass of the weak bosons due to the Higgs mechanism is

$$
\begin{aligned}
M_{W}^{2} & =\left(\frac{g_{2}}{2}\right)^{2} N_{D} F_{\pi}^{2}, \\
M_{Z}^{2} & =M_{W}^{2} / \cos ^{2} \theta_{W} .
\end{aligned}
$$

This means that the larger $N_{D}$ results smaller $F_{\pi}$.

The condensate $\langle\bar{T} T\rangle(T=U, D, N$, or $E)$ is related with the decay constant. If we assume the Euclidean mass function of the technifermion as

$$
\Sigma(x)=\left\{\begin{array}{cc}
m & x \leq m^{2} \\
0 & x>m^{2},
\end{array}\right.
$$

where $m$ is the typical scale of the dynamics, then we get

$$
\begin{aligned}
\left\langle\bar{T}_{L} T_{R}\right\rangle & =-\frac{N_{T C} m^{3}}{8 \pi^{2}}(1-\ln 2), \\
F_{\pi}^{2} & =\frac{N_{T C} m^{2}}{8 \pi^{2}}(2 \ln 2-1) .
\end{aligned}
$$

The condensate is calculated by the integration of the propagator, and the decay constant is calculated by using the Pagels-Stokar formula. We find the relation

$$
\begin{aligned}
\left\langle\bar{T}_{L} T_{R}\right\rangle & =-2 \pi F_{\pi}^{3} \sqrt{\frac{2}{N_{T C}}} \frac{1-\ln 2}{(2 \ln 2-1)^{3 / 2}} \\
& \simeq-2 \pi F_{\pi}^{3} .
\end{aligned}
$$

Similar relation $\langle\bar{T} T\rangle \simeq-4 \pi F_{\pi}^{3}$ is obtained from the argument of the naive dimensional analysis in the chiral perturbation theory[23].

To generate the mass of ordinary fermions, the technicolor interaction is extended. The multiplet of the extended gauge group should be very complicated one to generate the realistic mass spectrum. No one knows the realistic gauge group and representations. Here, we naively consider the extended gauge group $S U\left(N_{T C}+3\right)$ and the fundamental

\footnotetext{
${ }^{5}$ This is the naive relation. To tell the truth, the condensate should depend on the renormalization point, but the decay constant do not. We must use the technique of the operator product expansion for more rigorous arguments.
} 
representations as follows to explain the general idea of the mass generation.

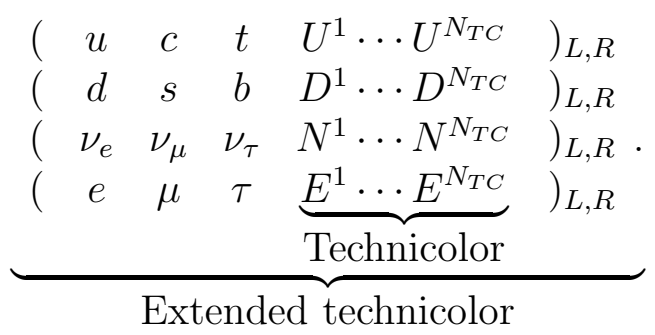

The right-handed neutrinos are introduced for simplicity. We assume the dynamics which hierarchically breaks the extended gauge group to the technicolor gauge group. Three hierarchical masses scales are assumed for each generations: $M_{1}, M_{2}$, and $M_{3}$. At the scale $M_{1}, S U\left(N_{T C}+3\right)$ breaks to $S U\left(N_{T C}+2\right)$, and the first generation is decoupled from the gauge multiples. Some gauge bosons get mass of order $M_{1}$ at this stage. It is the same for second and third generations. Then, the up-type quarks, for instance, get the following masses.

$$
\begin{aligned}
& m_{u}=\frac{g_{E T C}^{2}\left(M_{1}\right)}{M_{1}^{2}}\left\langle\bar{U}_{L} U_{R}\right\rangle \simeq \frac{g_{E T C}^{2}\left(M_{1}\right)}{M_{1}^{2}} 2 \pi F_{\pi}^{3} \\
& m_{c}=\frac{g_{E T C}^{2}\left(M_{2}\right)}{M_{2}^{2}}\left\langle\bar{U}_{L} U_{R}\right\rangle \simeq \frac{g_{E T C}^{2}\left(M_{2}\right)}{M_{2}^{2}} 2 \pi F_{\pi}^{3} \\
& m_{t}=\frac{g_{E T C}^{2}\left(M_{3}\right)}{M_{1}^{3}}\left\langle\bar{U}_{L} U_{R}\right\rangle \simeq \frac{g_{E T C}^{2}\left(M_{3}\right)}{M_{3}^{2}} 2 \pi F_{\pi}^{3} .
\end{aligned}
$$

The down-type quarks have the same mass spectrum with up-type, since we are considering the vector-like extended gauge group. So this naive model is not realistic. If we consider the flavor dependent condensation like

$$
\langle\bar{U} U\rangle \neq\langle\bar{D} D\rangle \neq\langle\bar{N} N\rangle \neq\langle\bar{E} E\rangle
$$

we can get the mass splitting in a weak doublet. But these splitting of the condensates cause the large effect on the $\rho$ parameter, in general. This will be discussed in the next chapter in detail.

Since the chiral symmetry of the technifermion is explicitly broken by the extended technicolor and the standard gauge group, some Nambu-Goldstone bosons in (4.15) become massive (pseudo-Nambu-Goldstone bosons) 9. These masses can evaluated by the Dashen's formula[24] []

$$
m_{\pi}^{2}=\frac{1}{F_{\pi}^{2}}\left\langle 0\left|\left[Q_{\pi},\left[Q_{\pi}, \mathcal{H}\right]\right]\right| 0\right\rangle,
$$

where $Q_{\pi}$ is the charge corresponding to the Nambu-Goldstone boson $\pi$, and $\mathcal{H}$ is the Hamiltonian density. But here, we briefly estimate it in the effective theoretical point of view 8 .

\footnotetext{
${ }^{6}$ The extended technicolor interaction and the standard strong and electroweak interactions are considered perturbatively.

${ }^{7}$ This formula is derived by using the current algebra and PCAC relation.

${ }^{8} \mathrm{~A}$ detailed estimation has been given in ref. 25.
} 
Figure 4.1: The diagrams for the mass of the Nambu-Goldstone bosons.

We can consider the coupling between the color octet Nambu-Goldstone bosons and gluons as

$$
\mathcal{L}_{e f f}=\operatorname{tr}\left\{\left(D_{\mu} \theta\right)^{\dagger}\left(D^{\mu} \theta\right)\right\}
$$

where

$$
\begin{aligned}
D_{\mu} \theta & =\partial_{\mu}-i g_{3}\left[G_{\mu}, \theta\right], \\
G_{\mu} & =G_{\mu}^{a} \frac{\lambda^{a}}{2} \\
\theta & =\theta^{a} \frac{\lambda^{a}}{2} .
\end{aligned}
$$

Namely, we assume the standard gauge coupling of the elementary colored scalar field. The mass is obtained by evaluating the diagram of fig.(4.1). Since the Nambu-Goldstone bosons are the composite particles, we assume the form factor as

$$
g_{3} \rightarrow g_{3} \cdot \frac{M_{V}^{2}}{M_{V}^{2}-k^{2}},
$$

where $k^{2}$ is the momentum of the gluon and $M_{V}$ is the mass of the vector boundstate composed by the technifermions which can mix with gluons. We get the value as

$$
M_{V}=m_{\rho} \frac{F_{\pi}}{f_{\pi}} \sqrt{\frac{3}{N_{T C}}} \simeq \sqrt{\frac{3}{N_{T C}}} \cdot 1 \mathrm{TeV}
$$

by the naive scaling up from the $\rho$ meson mass in QCD. Then, we get

$$
\begin{aligned}
m_{\theta}^{2} & \simeq \frac{9}{4 \pi} C_{2}(8) \alpha_{s} M_{V}^{2} \\
& \simeq(460 \mathrm{GeV})^{2} \frac{3}{N_{T C}}
\end{aligned}
$$

where $C_{2}(8)=N_{c}$ (Casimir coefficient), and we set $\alpha_{s}=0.1$. This is the same for color triplet Nambu-Goldstone bosons. We get

$$
\begin{aligned}
m_{T}^{2} & \simeq \frac{9}{4 \pi} C_{2}(3) \alpha_{s} M_{V}^{2} \\
& \simeq(300 \mathrm{GeV})^{2} \frac{3}{N_{T C}}
\end{aligned}
$$

The mass of the triplet is smaller than that of the octet, because of the difference of the Casimir coefficient. The mass of the charged Nambu-Goldstone bosons is also estimated in the same way by considering photon instead of gluon. The result is

$$
\begin{aligned}
m_{P^{ \pm}}^{2} & \simeq \frac{9}{4 \pi} \alpha M_{V}^{2} \\
& \simeq(70 \mathrm{GeV})^{2} \frac{3}{N_{T C}}
\end{aligned}
$$


Figure 4.2: The diagrams for the Yukawa coupling.

Figure 4.3: The diagrams for the masses of the quarks and leptons.

The mass of $P^{3}$ and $P^{0}$ is expected to be very small, since they do not couple with the gluon and photon. Only the explicit breaking of the chiral symmetry due to the weak interaction, the extended technicolor interaction, and the other additional interactions mediated by the massive particles is the small source of their mass. The explicit breaking due to the extended technicolor interaction is highly model dependent. If these masses can not become large, and the coupling of these bosons with the ordinary fermions (Yukawa coupling) is not small enough, this model is phenomenologically ruled out.

The Yukawa couplings between the pseudo-Nambu-Goldstone bosons and ordinary fermions are estimated by considering the diagram of fig.(4.2). We use the mass function of eq.(4.22), and the formula of Bethe-Salpeter amplitude

$$
F_{\pi} P^{a}(k, k)=2 i \Sigma\left(k^{2}\right) T^{a}
$$

which has already derived in the previous chapter. The coupling depend on the generation and flavor of the ordinary fermions as

$$
\left(g_{Y}^{a}\right)_{n}^{i j} F_{\pi}=N_{T C} \frac{g_{L}^{i} g_{R}^{j}}{16 \pi^{2}}\left(T^{a}\right)^{i j} \frac{m}{M_{n}^{2}} \int_{0}^{m^{2}} d k^{2} \frac{k^{2}}{m^{2}+k^{2}},
$$

where $i$ and $j$ are the indexes of the flavor and $n$ is the index of the generation. On the other hand, we get the mass of ordinary fermions by evaluation the diagram of fig.(4.3). We get

$$
m_{n}^{i j}=2 N_{T C} \frac{g_{L}^{i} g_{R}^{j}}{16 \pi^{2}} \frac{m}{M_{n}^{2}} \int_{0}^{m^{2}} d k^{2} \frac{k^{2}}{m^{2}+k^{2}} .
$$

Therefore he have the relation

$$
\left(g_{Y}^{a}\right)_{n}^{i j} F_{\pi}=2\left(T^{a}\right)^{i j} m_{n}^{i j}
$$

This is nothing but the Goldberger-Treiman relation ?. This formula is similar to the mass formula in the standard model, $m_{\psi}=g_{Y}^{\psi} v / \sqrt{2}$, where $v$ is the vacuum expectation value of the elementary Higgs field.

There is another important couplings for the pseudo-Nambu-Goldstone bosons. Since the axial current have the anomaly, in general, we have the coupling like in fig.(4.4) [27. This coupling is important for the production and decay of the pseudo-Nambu-Goldstone bosons. The strength of the coupling can be obtained as follows.

We have the anomalous Ward-Takahashi identity

$$
\partial^{\alpha} J_{\alpha}^{a}(x)=-(\delta \mathcal{L})^{a}+\frac{g^{(b)} g^{(c)} S^{a b c}}{16 \pi^{2}} \epsilon^{\mu \nu \rho \sigma} F_{\mu \nu}^{b}(x) F_{\rho \sigma}^{c}(x),
$$

\footnotetext{
${ }^{9}$ In QCD, the Yukawa coupling of the nucleons with the pions, $g_{\pi N}$, is given by the GoldbergerTreiman relation $g_{\pi N} f_{\pi}=2 g_{A} m_{N}$ [26], where $g_{A}$ is the axial vector coupling of nucleon and $m_{N}$ is the mass of nucleon.
} 
Figure 4.4: Coupling through the anomaly.

where $(\delta \mathcal{L})^{a}$ means the explicit breaking (See chapter 2), $F_{\mu \nu}^{b}$ and $F_{\rho \sigma}^{c}$ are the gauge field strength, $g^{(b)}$ and $g^{(c)}$ are the corresponding gauge coupling constant, and

$$
S^{a b c}=\frac{1}{2} \operatorname{tr}\left(T^{a}\left\{T^{b}, T^{c}\right\}\right)
$$

with the generators of the gauge interactions $T^{b}$ and $T^{c}(8 \times 8$ matrixes acting on the one family technifermions). We neglect the effect of the explicit breaking as the first approximation of the perturbation on it. Consider the matrix element

$$
\left\langle A^{b}\left(k_{1}\right) A^{c}\left(k_{2}\right)\left|\partial^{\alpha} J_{\alpha}^{a}(0)\right| 0\right\rangle=\frac{g^{(b)} g^{(c)} S^{a b c}}{16 \pi^{2}} \epsilon^{\mu \nu \rho \sigma}\left\langle A^{b}\left(k_{1}\right) A^{c}\left(k_{2}\right)\left|F_{\mu \nu}^{b}(0) F_{\rho \sigma}^{c}(0)\right| 0\right\rangle,
$$

where $A^{b}$ and $A^{c}$ are the gauge fields corresponding the the strength $F_{\mu \nu}^{b}$ and $F_{\rho \sigma}^{c}$, respectively. The right-hand side becomes

$$
i \int d^{4} e^{-i k x}[\text { right-hand side }]=(2 \pi)^{4} i \delta^{4}\left(k_{1}+k_{2}-k\right) \frac{g^{(b)} g^{(c)} S^{a b c}}{4 \pi^{2}} \epsilon_{\mu \nu \rho \sigma} \epsilon^{*}\left(k_{1}\right)^{\mu} \epsilon^{*}\left(k_{2}\right)^{\nu} k_{1}^{\rho} k_{2}^{\sigma}
$$

in the lowest order of the perturbation on the gauge interactions. To evaluate the left-hand side, we assume

$$
\partial^{\alpha} J_{\alpha}^{a} \simeq-F_{\pi} \square \Pi^{a},
$$

since the Nambu-Goldstone theorem says that the Nambu-Goldstone boson couple with the current as eq.(4.17). By assuming of this operator relation, the higher contribution, the axial vector resonance, for instance, is neglected. Therefore, the following result is valid only for the low energy pseudo-Nambu-Goldstone bosons. Then,

$$
\begin{aligned}
i \int d^{4} x e^{-i k x}[\text { left-hand side }] & \simeq-i F_{\pi} \int d^{4} x e^{-i k x} \square\left\langle A^{b}\left(k_{1}\right) A^{c}\left(k_{2}\right)\left|\Pi^{a}(x)\right| 0\right\rangle \\
& =-F_{\pi}\left\langle A^{b}\left(k_{1}\right) A^{c}\left(k_{2}\right) \mid \Pi^{a}(k)\right\rangle,
\end{aligned}
$$

where we used the Lehmann-Symanzik-Zimmermann reduction formula. Therefore, we obtain

$$
\left\langle A^{b}\left(k_{1}\right) A^{c}\left(k_{2}\right) \mid \Pi^{a}(k)\right\rangle=-(2 \pi)^{4} i \delta^{4}\left(k_{1}+k_{2}-k\right) \frac{g^{(b)} g^{(c)} S^{a b c}}{4 \pi^{2}} \epsilon_{\mu \nu \rho \sigma} \epsilon^{*}\left(k_{1}\right)^{\mu} \epsilon^{*}\left(k_{2}\right)^{\nu} k_{1}^{\rho} k_{2}^{\sigma},
$$

and the vertex of the diagram of fig.(4.4)

$$
\Gamma_{\mu \nu}^{a b c}\left(k_{1}, k_{2}\right)=-\frac{g^{(b)} g^{(c)} S^{a b c}}{4 \pi^{2}} \epsilon_{\mu \nu \rho \sigma} k_{1}^{\rho} k_{2}^{\sigma} .
$$

If the two gauge bosons are the same ones (for example, $\left\langle\gamma \gamma \mid \Pi^{a}\right\rangle$ ), the factor 2 must be multiplied.

Finally, we briefly review the experimental bound on the mass of the pseudo-NambuGoldstone bosons. The quarkonium can decay into the axions $P^{3}$ and $P^{0}$, through the anomaly induced vertex as fig.(4.5). From $\Upsilon(1 S)$ decay, we have the bound 
Figure 4.5: Quarkonium going to $P^{3} \gamma$ or $P^{0} \gamma$.

Figure 4.6: $\theta_{a}^{i}$ or $\theta_{a}$ production at the hadron collider.

$$
m_{P^{0}, P^{3}}>9 \mathrm{GeV}
$$

for the axions with decay constant $F_{\pi}=125 \mathrm{GeV}$ (one-family model) 28]. The colored Pseudo-Nambu-Goldstone bosons can be produced in hadron collider by the process in fig.(4.6). We have the bound

$$
m_{\theta}>86 \mathrm{GeV}
$$

for the color octet stable particles 29]. The leptoquarks, $T^{\alpha i}$ and $T^{\alpha}$, can be produced in $S$-channel at the $e p$ collider HERA. We have the bound 30]

$$
m_{T}>98 \sim 121 \mathrm{GeV} .
$$

The charged color singlet Pseudo-Nambu-Goldstone bosons, $P^{ \pm}$, can be produced in pair at LEP. We have the bound

$$
m_{P^{ \pm}}>41.7 \mathrm{GeV}
$$

as the bound on the charged Higgs 31]. 


\section{Chapter 5}

\section{Phenomenology of the Technicolor theory}

In this chapter we discuss the phenomenological bounds on the technicolor models. Flavorchanging neutral current (FCNC) problem is discussed in the first section. The solution of the problem by the large anomalous dimension dynamics is explained in the second section. The bound from the recent precision experiments is discussed in the third section. The prediction of the technicolor theory on the oblique correction $S, T$, and $U$ will be compared with the experiments. The bound through the non-oblique correction is also discussed in the fourth section.

\subsection{Flavor-changing neutral current problem}

In naive model of the extended technicolor theory, pseudo-Nambu-Goldstone bosons mediate large FCNC. The Yukawa couplings between the pseudo-Nambu-Goldstone bosons and the ordinary fermions depend on the models of the extended technicolor interaction. Consider the general two doublet Higgs system with Yukawa coupling

$$
\begin{aligned}
\mathcal{L}_{\text {Yukawa }} & =\left(g_{1}^{u}\right)^{i}{ }_{j} \bar{\psi}_{L i} \tilde{\Phi}_{1} u_{R}^{j}+\left(g_{2}^{u}\right)^{i}{ }_{j} \bar{\psi}_{L i} \tilde{\Phi}_{2} u_{R}^{j} \\
& +\left(g_{1}^{d}\right)^{i}{ }_{j} \bar{\psi}_{L i} \Phi_{1} d_{R}^{j}+\left(g_{2}^{d}\right)^{i}{ }_{j} \bar{\psi}_{L i} \Phi_{2} d_{R}^{j}+\text { h.c. } \\
& + \text { (lepton sector) },
\end{aligned}
$$

where $\tilde{\Phi}=i \tau^{2} \Phi^{*}$. If we consider the one-family model, the fields $\Phi_{1}$ and $\Phi_{2}$ can be identified as the fields which contain $\Pi^{i}$ and $P^{i}$, respectively. Assume that only $\Phi_{1}$ get the vacuum expectation value

$$
\left\langle\Phi_{1}\right\rangle=\frac{v}{\sqrt{2}}\left(\begin{array}{l}
0 \\
1
\end{array}\right) .
$$

Then, we get the quark mass matrixes

$$
\begin{aligned}
& \left(M_{u}\right)_{j}{ }_{j}=\left(g_{1}^{u}\right)^{i}{ }_{j} \frac{v}{\sqrt{2}} \\
& \left(M_{d}\right)_{j}{ }_{j}=\left(g_{1}^{d}\right)^{i}{ }_{j} \frac{v}{\sqrt{2}} .
\end{aligned}
$$


Figure 5.1: Example of FCNC: $K^{0}-\bar{K}^{0}$ mixing mediated by the pseudo-Nambu-Goldstone boson. The quarks with suffix $m$ denote the mass eigenstate.

These mass matrixes should be diagonalized to go to the mass eigenstate. It can be done by the bi-unitary chiral transformation

$$
\begin{aligned}
\left(U_{L}^{u}\right)^{\dagger} M_{u} U_{R}^{u} & =\left(\begin{array}{lll}
m_{u} & & \\
& m_{c} & \\
& & m_{t}
\end{array}\right), \\
\left(U_{L}^{d}\right)^{\dagger} M_{d} U_{R}^{d} & =\left(\begin{array}{lll}
m_{d} & & \\
& m_{s} & \\
& & m_{b}
\end{array}\right) .
\end{aligned}
$$

Though the Yukawa couplings of $\Phi_{1}\left(g_{1}^{u}\right.$ and $\left.g_{1}^{d}\right)$ become diagonal by this transformation, the Yukawa couplings of $\Phi_{2}\left(g_{2}^{u}\right.$ and $\left.g_{2}^{d}\right)$ do not always simultaneously become diagonal. Therefore, $\Phi_{2}$ can mediate the FCNC at tree level, in general. For example, it causes the large mixing of $K^{0}$ and $\bar{K}^{0}(|\Delta S|=2$ transition) as fig.(5.1). There exists FCNC in the standard model, but it is the small one loop effect suppressed by the GIM mechanism 32. The prediction of the standard model on the FCNC is consistent with the experiments. So, this tree level contribution is dangerous. The off diagonal element of the Yukawa couplings, $g_{2}^{u}$ and $g_{2}^{d}$, must be very small, or the mass of $\Phi_{2}$ is heavy. We have the constraint so that this tree level contribution is smaller than the contribution of the standard model. The stringent constraint comes from the $K^{0}-\bar{K}^{0}$ mixing

$$
\left(\frac{\left(g_{Y}^{d}\right)_{s d}}{M_{P}}\right)^{2}<10^{-12} \mathrm{GeV}^{-2} .
$$

It is naturally expected that $\left(g_{Y}^{d}\right)_{s d}$ is of the order of the Yukawa coupling of the strange quark $g_{Y}^{s}=\sqrt{2} m_{s} / v \simeq 5 \times 10^{-4}$, where $m_{s} \simeq 83 \mathrm{MeV}$ (at $300 \mathrm{GeV}$ ). Then we get

$$
M_{P}>500 \mathrm{GeV}
$$

We must naturally explain why the off diagonal elements of the Yukawa couplings are so small, if the masses of the pseudo-Nambu-Goldstone bosons are smaller than a few hundred $\mathrm{GeV}$. In the naive model, many pseudo-Nambu-Goldstone bosons are possibly lighter than a few $\mathrm{GeV}$, which is estimated in previous chapter.

The gauge bosons of the extended technicolor interaction can also mediate the FCNC. The massive gauge boson exchanges generate the effective four fermion interactions like fig. (5.2a), where quarks are at the eigenstate of the gauge interactions. These four fermion interactions become the FCNC interaction, when we go to the mass eigenstate of the quarks. The four fermion interaction of fig.(5.2 a) results $K^{0}-\bar{K}^{0}$ mixing as fig.(5.2 $\left.\mathrm{b}\right)$. We have the constraint for the mass and coupling of the gauge boson

$$
\left(\frac{g_{E T C} \sin \theta_{c}}{M}\right)^{2}<10^{-12} \mathrm{GeV}^{-2}
$$


Figure 5.2: Example of FCNC: $K^{0}-\bar{K}^{0}$ mixing mediated by the extended technicolor gauge bosons. (a) The process in gauge eigenstate. (b) The same process in mass eigenstate. The Cabibbo angle $\theta_{c}$ is taken as the typical mixing angle.

Since $\sin ^{2} \theta_{c} \simeq 1 / 20$, we obtain

$$
\left(\frac{g_{E T C}}{M}\right)^{2}<(200 \mathrm{TeV})^{-2}
$$

The squared coupling $g_{E T C}^{2}$ is the largest one in $g_{R}(M)^{2}, g_{L}(M)^{2}$, and $g_{R}(M) \times g_{L}(M)$, where $g_{L}$ and $g_{R}$ are the extended technicolor effective gauge couplings for the left-handed fermions and the right-handed fermions, respectively.

The mass of the gauge boson $M$ likely to be equal to the mass of the sideways boson which is related with the strange quark mass Đ. Namely, we have

$$
m_{s}=\frac{g_{L}(M) g_{R}(M)}{M^{2}} 2 \pi F_{\pi}^{3} \text {. }
$$

Therefore we get

$$
\frac{g_{L}(M) g_{R}(M)}{M^{2}}=\frac{m_{s}}{2 \pi F_{\pi}^{3}} \simeq(10 \mathrm{TeV})^{-2},
$$

where we use $F_{\pi}=125 \mathrm{GeV}$ by assuming the one-family model. There are about three order excess from the bound of eq.(5.10).

In general, in the naive extended technicolor model, there are large additional FCNCs which are mediated by the pseudo-Nambu-Goldstone bosons and the extended technicolor gauge bosons. This is called FCNC problem in the extended technicolor theory [34]. Of course, we can consider the complicated models to avoid FCNCs: complicated representation of the extended technicolor multiplet, complicated breaking pattern of the extended technicolor gauge group, etc. But it is unnatural. Technicolor theory had once died of this problem. But it was pointed out that if we consider the special dynamics for the technicolor, the masses of the pseudo-Nambu-Goldstone bosons and the extended technicolor gauge bosons can be large without the change of the masses of the ordinary fermions and weak bosons. The dynamics is called the large anomalous dimension dynamics 10. In next section, we explain how the dynamics solves the problem.

\subsection{Technicolor with large anomalous dimension}

We know that the non-Abelian gauge theory has the nature of the asymptotic freedom. The gauge interaction goes down at the high energy. This is the reason that the mass function of the technifermion dumps as $\Sigma(x) \sim 1 / x$ for large Euclidean squared momentum $x=p^{2}$. If the technicolor interaction does not quickly go down at the high energy,

\footnotetext{
${ }^{1}$ In the naive one-family model in the previous section, the sideways bosons of the strange quark is associated with the broken off diagonal generator in $S U\left(N_{T C}+2\right) \rightarrow S U\left(N_{T C}+1\right)$ breaking. The gauge boson which mediates FCNC is associated with the broken diagonal generator of the breaking.
} 
Figure 5.3: The diagram for the mass function of the ordinary fermion.

the dump of the mass function becomes slow as

$$
\Sigma(x) \sim \frac{1}{x}\left(\frac{x}{m_{T}^{2}}\right)^{\gamma_{m} / 2}
$$

with the anomalous dimension $\gamma_{m}$, where $m_{T}$ is the typical scale of the dynamics (Remember the discussion in section 3.1 and eq.(3.81).).

Two kind of explicit models with large anomalous dimension are proposed. One is the gauge theory with slowly running coupling (walking coupling)[11], another is the system of the gauge plus strong four fermion interaction (gauged Nambu-Jona-Lasinio model)[12]. Walking dynamics can realize the large anomalous dimension $\gamma_{m} \simeq 1$. More large anomalous dimension $\gamma_{m} \simeq 2$ can be realized in the gauged Nambu-Jona-Lasinio model, since the four fermion interaction does not go down at high energy.

As we will explain in the following, if the anomalous dimension is large, the masses of the ordinary fermions and the pseudo-Nambu-Goldstone bosons are extensively enhanced, while the decay constant of the Nambu-Goldstone boson (mass of the weak boson) is unchanged. Enhancement of the ordinary fermion mass allows us to consider the heavy extended technicolor gauge bosons. This is the idea to solve the FCNC problem by the large anomalous dimension dynamics 10.

The mass function of the ordinary fermions can be calculated as (fig.(5.3))

$$
\Sigma^{f}(q)=-N_{T C} \int \frac{d^{4} p}{(2 \pi)^{4} i} \frac{g_{E T C}}{\sqrt{2}} \gamma_{\mu} \frac{1-\gamma_{5}}{2} S(p) \frac{1-\gamma_{5}}{2} \frac{g_{E T C}}{\sqrt{2}} \gamma_{\nu} D^{\mu \nu}(q-p),
$$

where $S(p)$ is the technifermion full propagator, $g_{E T C}$ is the sideways gauge coupling, and $D^{\mu \nu}(q-p)$ is the sideways massive gauge boson propagator. In section 3.1, we estimated the full technifermion propagator at the large momentum as

$$
\begin{gathered}
\lim _{p \rightarrow \infty} \frac{1-\gamma_{5}}{2} S(p) \frac{1-\gamma_{5}}{2}=i \lim _{p \rightarrow \infty} U_{\bar{T} T}(p, g, \mu) \frac{1-\gamma_{5}}{2}\left\langle 0\left|\bar{T}_{R}(0) T_{L}(0)\right| 0\right\rangle, \\
\lim _{p \rightarrow \infty} U_{\bar{T} T}(p, g, \mu)=c \frac{1}{i} \frac{3}{2 N_{T C}} g^{2} C_{2} \frac{1}{p^{4}}\left(\frac{p^{2}}{\mu^{2}}\right)^{\gamma_{m} / 2},
\end{gathered}
$$

where we take the Landau gauge in the technicolor interaction (see eqs.(3.65) and (3.78).). We get the ordinary fermion mass which is defined by $m_{f} \equiv \frac{1}{4} \operatorname{tr}\left(\Sigma^{f}(q=0)\right)$ as

$$
\begin{aligned}
m_{f} \simeq & -\frac{1}{4} N_{T C} \operatorname{tr} \int \frac{d^{4} p}{(2 \pi)^{4} i} \frac{g_{E T C}}{\sqrt{2}} \gamma_{\mu} \cdot i \cdot c \frac{1}{i} \frac{3}{2 N_{T C}} g^{2} C_{2} \frac{1}{p^{4}}\left(\frac{p^{2}}{m_{T}^{2}}\right)^{\gamma_{m} / 2} \\
& \times \frac{1-\gamma_{5}}{2} \frac{m_{T}^{3}}{2} \frac{g_{E T C}}{\sqrt{2}} \gamma_{\nu} \frac{g^{\mu \nu}}{p^{2}-M_{f}^{2}} \\
\simeq & \frac{3 c}{64 \pi^{2}}(-1)^{\gamma_{m} / 2} g_{E T C}^{2} g^{2} C_{2} \frac{m_{T}^{3}}{M_{f}^{2}} \int_{0}^{M_{f}^{2}} d x \frac{1}{x}\left(\frac{x}{m_{T}^{2}}\right)^{\gamma_{m} / 2} \\
= & C \frac{g_{E T C}^{2} m_{T}^{3}}{2 M_{f}^{2}}\left(\frac{M_{f}}{m_{T}}\right)^{\gamma_{m}}
\end{aligned}
$$


Figure 5.4: Diagram for the mass of pseudo-Nambu-Goldstone bosons.

where we set $\langle 0|\bar{T}(0) T(0)| 0\rangle=m_{T}^{3}$ and $\mu=m_{T}, M_{f}$ is the mass of the sideways gauge bosons, and

$$
C=\frac{3 c}{32 \pi} g^{2} C_{2}(-1)^{\gamma_{m} / 2} B\left(2-\gamma_{m} / 2,2-\gamma_{m} / 2\right) \frac{\pi}{\sin \pi \gamma_{m} / 2}
$$

which is expected to be of the order unity $(B(p, q)$ is the beta function). The naive estimation in the previous chapter by the mean field approximation

$$
m_{f} \simeq \frac{g_{E T C}^{2}}{M_{f}^{2}}\left|\left\langle\bar{T}_{L} T_{R}\right\rangle\right|
$$

corresponds to the case $\gamma_{m}=0$ (QCD-like dynamics). If the running coupling of the technicolor interaction is constant (as an extreme limit of the walking dynamics), we get the asymptotic behavior of the technifermion mass function as $\Sigma(x) \sim 1 / \sqrt{x}$, and get $\gamma_{m}=1$ (Remember the discussion in the last of the section 3.1.). In that case, the mass of the ordinary fermion is proportional to $1 / M_{f}$ instead of $1 / M_{f}^{2}$. Namely, the mass of the ordinary fermion is enhanced by the anomalous dimension. Therefore, heavier sideways gauge bosons are allowed. The mass of the sideways bosons which is related with the strange quark mass becomes

$$
\frac{M_{2}}{g_{E T C}^{2}} \simeq \frac{\left(4 \pi F_{\pi}^{3}\right)^{2 / 3}}{2 m_{s}} \simeq 510 \mathrm{TeV} .
$$

The bound from the $K^{0}-\bar{K}^{0}$ mixing, eq.(5.10), can be satisfied if $g_{E T C}$ is of the order of unity.

The masses of the pseudo-Nambu-Goldstone bosons are enhanced by this dynamics. The mass can be estimated by considering the diagram of fig.(5.4). The diagram denotes that the explicit chiral symmetry breaking by the gauge interactions (strong, electromagnetic, weak, extended technicolor interaction, or the others which are spontaneously broken) makes the mass of the Nambu-Goldstone boson. Consider the case that the chiral symmetry is explicitly broken by the additional gauge interaction which is spontaneously broken, for example. This is the case for the axions, $P^{3}$ and $P^{0}$, in the one-family model (Corresponding chiral symmetries are broken by the Pati-Salam gauge interaction [33].). The mass can be estimated as

$$
\begin{gathered}
M_{a}^{2}=-\operatorname{tr} \int \frac{d^{4} p}{(2 \pi)^{4} i} \frac{d^{4} k}{(2 \pi)^{4} i} P^{a}(p-k, p-k) \frac{1}{-(\not p-\not k)} g \mathcal{T}^{\alpha} \gamma_{\mu} \frac{1}{-\not p} \\
\times P^{a}(p, p) \frac{1}{-\not p} g \mathcal{T}^{\beta} \gamma_{\nu} \frac{1}{-(\not p-\not k)} \frac{\delta_{\alpha \beta} g^{\mu \nu}}{-M^{2}} .
\end{gathered}
$$

The index $a$ is not summed over. Since the Bethe-Salpeter amplitude at the zero momentum transfer can be written by the mass function of the technifermion as

$$
P^{a}(p, p)=2 i \gamma_{5} T^{a} \frac{\Sigma(p)}{F_{\pi}},
$$


we obtain

$$
M_{a}^{2} \simeq C \operatorname{tr}\left(\left[T^{a}, \mathcal{T}^{\alpha}\right]\left[T^{a}, \mathcal{T}^{\alpha}\right]\right) g^{2} \frac{m_{T}^{4}}{F_{\pi}^{2}} \frac{m_{T}^{2}}{M^{2}}\left(\frac{M^{2}}{m_{T}^{2}}\right)^{\gamma_{m}}
$$

by using

$$
\Sigma(x) \simeq \frac{m_{T}^{3}}{x}\left(\frac{x}{m_{T}^{2}}\right)^{\gamma_{m} / 2}
$$

in Euclidean space, where $C$ is the dimensionless constant which is expected to be of the order of unity, and the trace of the generators should not be zero if the considering gauge interaction breaks the chiral symmetry $\%$. If the anomalous dimension is small, the squared mass is proportional to the small factor $m_{T}^{2} / M^{2}$. But if $\gamma_{m}=1$, the suppression by $M$ is disappeared. Suppose that $M=350 \mathrm{TeV}$. Then, the mass of the pseudoNambu-Goldstone boson is

$$
M^{2} \simeq \frac{1}{F_{\pi}^{2}} \cdot \frac{\left(4 \pi F_{\pi}^{3}\right)^{2}}{(350 \mathrm{TeV})^{2}} \simeq(560 \mathrm{MeV})^{2}
$$

for $\gamma_{m}=0$, but

$$
M^{2} \simeq \frac{1}{F_{\pi}^{2}} \cdot\left(4 \pi F_{\pi}^{3}\right)^{4 / 3} \simeq(680 \mathrm{GeV})^{2}
$$

for $\gamma_{m}=1$, where we set $m_{T}^{3}=4 \pi F_{\pi}^{3}$ and $F_{\pi}=125 \mathrm{GeV}$ (one family model). The pseudo-Nambu-Goldstone bosons become heavy, and the FCNCs mediated by them are suppressed.

The order of the decay constant of the Nambu-Goldstone boson is not affected whether the anomalous dimension is large or not. We can estimate the decay constant by using the Pagels-Stokar formula (eq.(3.99))

$$
F_{\pi}^{2}=\frac{N_{T C}}{4 \pi^{2}} \int d x \frac{x \Sigma(x)}{\left(\Sigma^{2}(x)+x\right)^{2}}\left\{\Sigma(x)-\frac{1}{2} x \frac{d \Sigma(x)}{d x}\right\} .
$$

Consider the mass function

$$
\Sigma(x)=\left\{\begin{array}{cc}
m_{T} & x \leq m_{T}^{2} \\
\frac{m_{T}^{3}}{x}\left(\frac{x}{m_{T}^{2}}\right)^{\gamma_{m} / 2} & x>m_{T}^{2}
\end{array}\right.
$$

Then we obtain

$$
F_{\pi}^{2}=\frac{N_{T C}}{8 \pi^{2}} m_{T}^{2}\left\{1+\frac{1}{2} \cdot \frac{3-\gamma_{m} / 2}{1-\gamma_{m} / 2}\right\}
$$

As far as the anomalous dimension $\gamma_{m}$ is not extremely near 2 , the variation of the anomalous dimension does not change the order of the decay constant. The reason is that the integral of the Pagels-Stokar formula is infrared dominant. The decay constant is the quantity which is determined mainly by the low energy behavior of the dynamics.

\footnotetext{
${ }^{2}$ The contribution which is proportional to $\operatorname{tr}\left(T^{a} T^{a} \mathcal{T}^{\alpha} \mathcal{T}^{\alpha}\right)$ comes from the other diagrams, since $\operatorname{tr}\left(\left[T^{a}, \mathcal{T}^{\alpha}\right]\left[T^{a}, \mathcal{T}^{\alpha}\right]\right)=2 \operatorname{tr}\left(T^{a} \mathcal{T}^{\alpha} T^{a} \mathcal{T}^{\alpha}\right)-2 \operatorname{tr}\left(T^{a} T^{a} \mathcal{T}^{\alpha} \mathcal{T}^{\alpha}\right)$

${ }^{3}$ The experimental lower bound on the mass of the Pati-Salam gauge boson 35. Pati-Salam gauge interaction in the technifermion sector breaks the chiral symmetries which are related with the axions.
} 
Figure 5.5: Oblique correction.

Figure 5.6: Corrections due to the Higgs. (a) Oblique correction. (b) Vertex correction. (c) Box diagram.

In this way, the FCNC problem can be solved by considering the large anomalous dimension dynamics for the technicolor interaction. The walking gauge coupling is obtained by assuming large number of technifermions (large $N_{F}$ ) so that the $\beta$-function becomes very small. But it should be noted that the number of the pseudo-Nambu-Goldstone bosons increases together with the number of the technifermions. The large number of pseudo-Nambu-Goldstone bosons are dangerous for FCNC, even if they are heavy. And it should be noted that the value of the decay constant decreases as the increasing of the number of the technifermion weak doublets. We know that the small value of the decay constant means the light pseudo-Nambu-Goldstone bosons.

In the gauged Nambu-Jona-Lasinio model, the four fermion interaction must be the effective interaction which is mediated by some massive particles, since the four fermion interaction is not renormalizable f Someone consider that the origin of the strong four fermion interaction is the extended technicolor interaction. But it is not clear that such strong four fermion interaction can be realized as the low energy effective interaction of the gauge theory . This is the main topics of this thesis, namely, whether the effective strong four fermion interaction is possible or not in the tumbling gauge theory which is a candidate of the dynamics of the extended technicolor theory. We will discuss this topics in and after the next chapter.

\subsection{Technicolor and precision experiments I}

The electroweak theory is precisely tested on the Z-pole at LEP. The experiments with extremely high precision makes possible to test the one-loop radiative correction. Since the new physics beyond the standard model affects on the low energy phenomena through the radiative correction, we can obtain some constraint on the new physics. Recently, the radiative correction on the electroweak interaction due to the new physics is parametrized by three parameters, $S, T$, and $U$ [37]. These three parameters describe so called oblique correction due to the new physics which is characterized by a certain high energy scale in comparison with the electroweak scale.

The radiative correction on the self-energies of the electroweak gauge bosons is called oblique correction (fig.(5.5)). In the standard model the non-oblique correction is suppressed by the factor $m_{f} / M_{Z}$ relative to the oblique correction, where $m_{f}$ is the mass of the quarks and leptons. Consider the radiative correction on the four fermion process due to the Higgs field (fig.(5.6)). The order of the oblique correction of fig.(5.6a) to the

\footnotetext{
${ }^{4}$ Recently, it is shown that the system of the gauge plus four fermion interaction can be renormalizable in ladder approximation [36].

${ }^{5}$ We do not consider the gauge symmetry breaking by the vacuum expectation value of the elementary scalar fields.
} 
Figure 5.7: Non-oblique correction due to the top quark.

amplitude is $g_{2}^{2}\left(g_{2} / \sqrt{2} \cos \theta\right)^{2}$, where $\theta$ is the Weinberg angle. The factor $\left(g_{2} / \sqrt{2} \cos \theta\right)^{2}$ comes from the Z-Higgs coupling. The order of the vertex correction of fig.(5.6) b) is $g_{2}^{2}\left(g_{2} / \sqrt{2} \cos \theta\right)^{2}\left(m_{f} / M_{Z}\right)^{2}$. The factor $m_{f} / M_{Z}$ comes from the Yukawa coupling $g_{Y}^{f}=$ $\sqrt{2} m_{f} / v$ and $M_{Z}=g_{2} v / 2 \cos \theta$, where $v / \sqrt{2}$ is the vacuum expectation value of the Higgs field. The contribution of the box diagram fig.(5.6c) is also suppressed by the same factor. The order of the contribution is $\left(g_{2} / \sqrt{2} \cos \theta\right)^{4}\left(m_{f} / M_{Z}\right)^{4}$. Essentially, as far as we consider the light quarks and light leptons as the incoming and the outgoing particle, the non-oblique correction can be neglected. But the heavy top quark can generate the large non-oblique correction, even if it does not appear as the incoming and outgoing particle (Now we know that the top quark is heavier than the Z-boson.). For example, consider the contributions of the diagrams of fig.(5.7). These non-oblique correction should be considered.

If we consider the new physics which is characterized by the very high energy scale $M_{\text {new }} \gg M_{Z}$, the oblique correction is parametrized only by three parameters. There are four kind of vacuum polarization of gauge bosons in the electroweak theory: $\Pi_{11}^{\mu \nu}(q)=$ $\Pi_{22}^{\mu \nu}(q), \Pi_{33}^{\mu \nu}(q), \Pi_{Y Y}^{\mu \nu}(q), \Pi_{3 Y}^{\mu \nu}(q)$, where indexes 1, 2, and 3 mean the $S U(2)_{L}$ gauge bosons, and index $Y$ means the $U(1)_{Y}$ gauge boson. The $U(1)_{e m}$ symmetry ensures the relation $\Pi_{11}^{\mu \nu}(q)=\Pi_{22}^{\mu \nu}(q)$. The new physics contribution is defined by subtracting the contribution of the standard model

$$
\Pi_{\text {new }}^{\mu \nu}(q)=\Pi_{\text {full }}^{\mu \nu}(q)-\Pi_{S M}^{\mu \nu}\left(q ; m_{t}, M_{H}\right) .
$$

Since the masses of the top quark and Higgs are unknown, we must fix the masses as an reference point of the subtraction. The true value of these masses may be different from the reference value. The difference is treated as the contribution of the new physics. We simply write $\Pi_{n e w}^{\mu \nu}(q)$ as $\Pi^{\mu \nu}(q)$ from now on.

If the scale of the new physics $M_{\text {new }}$ is sufficiently large, four $\Pi^{\mu \nu}(q)$ can be expended as

$$
\begin{aligned}
\Pi^{\mu \nu}(q) & =\left(g^{\mu \nu}-\frac{q^{\mu} q^{\nu}}{q^{2}}\right) \Pi\left(q^{2}\right) \\
& =\left(g^{\mu \nu}-\frac{q^{\mu} q^{\nu}}{q^{2}}\right)\left(\Pi(0)+q^{2} \Pi^{\prime}(0)+O\left(q^{2} / M_{\text {new }}^{2}\right)\right) .
\end{aligned}
$$

Up to the order $q^{2} / M_{\text {new }}^{2}$, the correction to the Lagrangian becomes as follows.

$$
\begin{aligned}
\mathcal{L}_{\text {gauge }}= & -\frac{1}{4}\left(1-g_{2}^{2} \Pi_{11}^{\prime}(0)\right) W_{1}^{\mu \nu} W_{1 \mu \nu}+\frac{1}{2} g_{2}^{2}\left(\frac{v^{2}}{4}+\Pi_{11}(0)\right) W_{1}^{\mu} W_{1 \mu} \\
& +\left(W_{2} \rightarrow W_{1}\right) \\
& -\frac{1}{4}\left(1-g_{2}^{2} \Pi_{33}^{\prime}(0)\right) W_{3}^{\mu \nu} W_{3 \mu \nu} \\
& -\frac{1}{4}\left(1-g_{1}^{2} \Pi_{Y Y}^{\prime}(0)\right) B^{\mu \nu} B_{\mu \nu}
\end{aligned}
$$




$$
\begin{aligned}
& +\frac{1}{2} \frac{4 \pi \alpha}{s c} \Pi_{3 Y}^{\prime}(0) W_{3}^{\mu \nu} B_{\mu \nu} \\
& +\frac{1}{2}\left(\begin{array}{ll}
W_{3 \mu} & B_{\mu}
\end{array}\right)\left(\frac{v^{2}}{4}+\Pi_{33}(0)\right)\left(\begin{array}{cc}
g_{2}^{2} & -g_{1} g_{2} \\
-g_{1} g_{2} & g_{1}^{2}
\end{array}\right)\left(\begin{array}{c}
W_{3}^{\mu} \\
B^{\mu}
\end{array}\right),
\end{aligned}
$$

where $\alpha$ is the fine structure constant, and $s$ and $c$ are the sine and cosine of the Weinberg angle, respectively. Here, we used the relations

$$
\begin{aligned}
\Pi_{3 Y}(0)+\Pi_{33}(0) & =0 \\
\Pi_{3 Y}(0)+\Pi_{Y Y}(0) & =0
\end{aligned}
$$

which come from the $U(1)_{e m}$ symmetry. There are six parameters ( $\Pi$ s and $\Pi^{\prime} s$ ) in the Lagrangian. Three of the six parameters can be absorbed into the gauge couplings and vacuum expectation value as

$$
\begin{aligned}
\Pi_{11}^{\prime}(0) & \rightarrow W \text { fields } \rightarrow g_{2}, \\
\Pi_{Y Y}^{\prime}(0) & \rightarrow B \text { field } \rightarrow g_{1}, \\
\Pi_{11}(0) & \rightarrow v
\end{aligned}
$$

Therefore, these are not the physical quantities. After that, we obtain

$$
\begin{aligned}
& \mathcal{L}_{\text {gauge }}=-\frac{1}{4}\left(W_{1}^{\mu \nu} W_{1 \mu \nu}+W_{2}^{\mu \nu} W_{2 \mu \nu}\right) \\
& +\frac{1}{2} g_{2}^{2} \frac{v^{2}}{4}\left(W_{1}^{\mu} W_{1 \mu}+W_{2}^{\mu} W_{2 \mu}\right) \\
& -\frac{1}{4}\left(1+\frac{\alpha}{4 s^{2}} U\right) W_{3}^{\mu \nu} W_{3 \mu \nu} \\
& -\frac{1}{4} B^{\mu \nu} B_{\mu \nu} \\
& -\frac{1}{4} \frac{\alpha}{2 s c} S W_{3}^{\mu \nu} B_{\mu \nu} \\
& +\frac{1}{2}\left(\begin{array}{ll}
W_{3 \mu} & B_{\mu}
\end{array}\right) \frac{v^{2}}{4}(1-\alpha T)\left(\begin{array}{cc}
g_{2}^{2} & -g_{1} g_{2} \\
-g_{1} g_{2} & g_{1}^{2}
\end{array}\right)\left(\begin{array}{c}
W_{3}^{\mu} \\
B^{\mu}
\end{array}\right),
\end{aligned}
$$

where

$$
\begin{aligned}
S & \equiv-16 \pi \Pi_{3 Y}^{\prime}(0) \\
T & \equiv \frac{4 \pi}{s^{2} c^{2} M_{Z}^{2}}\left[\Pi_{11}(0)-\Pi_{33}(0)\right] \\
U & \equiv 16 \pi\left[\Pi_{11}^{\prime}(0)-\Pi_{33}^{\prime}(0)\right]
\end{aligned}
$$

$S$ denotes the $B-W_{3}$ kinetic mixing, $T$ denotes the breaking of the custodial symmetry [38] (The deviation of the $\rho$ parameter from unity), and $U$ also denotes the breaking of the custodial symmetry. $U$ is the next order of the $q^{2} / M_{\text {new }}^{2}$ expansion in comparison with $T$. These three parameters, $S, T$, and $U$, are the physical parameters which values should be restricted by the experiments. 
Figure 5.8: One-loop diagrams for the oblique corrections. (a) $S$ parameter. (b) $T$ (and $U)$ parameter.

The physical quantities can be written with the new physics contribution. For example, the total width of the Z-boson is obtained as

$$
\Gamma_{Z}=\Gamma_{Z}^{S M}+a S+b T
$$

where $\Gamma_{Z}^{S M}$ is the prediction of the standard model and

$$
\begin{gathered}
a=-\frac{\alpha^{2} M_{Z}}{12 s^{2} c^{2}\left(c^{2}-s^{2}\right)} \sum_{f}\left(I_{3 f}-s^{2} Q_{f}\right) Q_{f} N_{f}, \\
b=\frac{\alpha^{2} M_{Z}}{6 s^{2} c^{2}} \sum_{f}\left\{\left(I_{3 f}-s^{2} Q_{f}\right)^{2} N_{f}+\frac{2 s^{2} c^{2}}{c^{2}-s^{2}}\left(I_{3 f}-s^{2} Q_{f}\right) Q_{f} N_{f}\right\},
\end{gathered}
$$

where $I_{3 f}, Q_{f}$, and $N_{f}$ denote weak isospin, electric charge, and effective number of color with respect to the fermion of flavor $f$, respectively [37]. The measured total width with small error restricts the values of $S$ and $T$ to a band in $S-T$ plane. The $U$ parameter appears only in $M_{W} / M_{Z}$, if we consider only the physical quantities which have already precisely measured. Therefore, $U$ parameter is not important at present.

Technicolor theory predicts the existence of the pseudo-Nambu-Goldstone bosons and the vector boundstates (techni- $\rho$ ) at high energy. These particle contribute to the $S, T$ (and $U$ ) parameters. Now, we briefly estimate the prediction of the technicolor.

Calculation of the one-loop diagram of fig.(5.8 a) gives

$$
S=\frac{N_{T C}}{6 \pi} \sum_{\text {doublets }}\left(1-Y_{L} \ln \frac{m_{N}^{2}}{m_{E}^{2}}\right),
$$

where $m_{N}$ and $m_{E}$ are the masses of the technifermions of weak isospin $1 / 2$ and $-1 / 2$, respectively, and $Y_{L}$ is the hypercharge of the weak doublet. The summention is taken over all the weak doubles. The contribution of the one doublet is $S \simeq 0.05 N_{T C}$, if the masses are degenerate in the doublet. The non-perturbative effect of the technicolor interaction is not included in this estimation. But we know the tendency that $S$ is proportional to the number of the techni-doublet and $N_{T C}$. Namely, large technicolor sector results large value of $S$. The difference of the mass in the weak doublet can not be the large source of the change of the value of $S$. The dependence is not the power but only logarithmic, since $S$ denotes the kinetic mixing of $W_{3}$ and $B$.

If the dynamics of the technicolor is postulated as the QCD-like one, we can estimate the value of $S$ including the non-perturbative effect by scaling up in energy. The boundstates due to QCD, $\pi, \rho$ and $a_{1}$ mason and so on, contribute to the vacuum polarization $\Pi_{3 Y}^{\mu \nu}$. The contribution of the techni- $\rho$ and techni- $a_{1}$ and so on is obtained by scaling up the experimental data in the low energy to the high energy. The value

$$
S \simeq 0.1 N_{T C} N_{D}
$$


Figure 5.9: Bound form the experiments and the prediction of the technicolor theory. Technicolor theory predicts the value of the right-hand region from the vertical line (prediction of the $S U(2)_{T C}$ one-family model).

is obtained [37], where $N_{D}$ is the number of the doublet. This value is about two times larger than the value from the above naive estimation. In the technicolor theory with large anomalous dimension, it is expected that $0.05<S / N_{T C} N_{D}<0.1$. Namely, $S / N_{T C} N_{D}$ will take the value between the values of the above two estimation, since the constant technifermion mass in naive estimation is expected to be the case $\gamma_{m}=2$ (For numerical estimation, see ref.[39].).

The naive estimation of $T$ parameter by the calculation of the one-loop diagram of fig. (5.8b) gives

$$
T=\frac{N_{T C}}{16 \pi s^{2} c^{2} M_{Z}^{2}} \sum_{\text {doublets }}\left[m_{N}^{2}+m_{E}^{2}-\frac{2 m_{N}^{2} m_{E}^{2}}{m_{N}^{2}-m_{E}^{2}} \ln \frac{m_{N}^{2}}{m_{E}^{2}}\right] .
$$

This is the same formula for $\delta \rho=\rho-1\left(\delta \rho^{\text {new }}=\alpha T\right)$. The difference of the masses in a doublet cause the large effect, since the dependence is in power. If we consider the technicolor model in which the custodial symmetry is not broken in the technifermion sector (equal values of the condensates in a doublet), $T$ parameter can be very small. Only the effect of the heavy extended technicolor bosons makes the deviation of $T$ from zero.

Now, we compare the above estimation with the bound from the experiments. Especially, the experimental restriction on the value of $S$ is important, since $S$ reflects the "size" of the technicolor sector. It should also be noted that the experiments can directly restrict the technicolor sector itself. This is not the test of the extended technicolor theory, but the technicolor theory. The bound from FCNC which is discussed in the previous section is the model dependent one, since FCNC strongly depends on the model-dependent extended technicolor interaction.

The bound from the experiment is displayed in fig.(5.9) with the prediction of the technicolor theory. The reference values of the masses of the top quark and Higgs are taken as $m_{t}=150 \mathrm{GeV}$ and $M_{H}=1 \mathrm{TeV}$. We use the following experiments: total width of Z-boson, R-ratio at the Z-pole, b quark forward-backward asymmetry, $\tau$ polarization asymmetry, the left and right couplings in the deep inelastic neutrino scattering, and atomic parity violation of ${ }_{53}^{133} \mathrm{Cs}$.40. We see that the technicolor theory predicts too large $S$, and it is disfavored by the experiments. The technicolor theory with large technicolor sector (large $N_{T C}, N_{D}$ ) is strongly disfavored by the experiments.

At present, there are no definite solution of this difficulty. The mass difference in the doublets of the technifermions can give the negative contribution to $S$. But if we set the mass difference so that the $S$ parameter becomes small enough, the value of $T$ parameter becomes too large to be consistent with the experiments. It is difficult to simultaneously suppress $S$ and $T$ parameters. It is pointed out that the Majorana technifermions can give the negative contribution to the $S$ parameter [41] without drastical changing of the $T$ parameter. But the contribution is very small $\left(\delta S \simeq-10^{-2}\right.$ and $\delta T \simeq-10^{-3}$ at every set 
with two Majorana technifermions.), and the many Majorana technifermion are needed to be consistent with the experiment.

We can consider the special dynamics to avoid this difficulty. The large anomalous dimension dynamics and the dynamics of the chiral gauge theory are the candidates. But it is the difficult work to find the good dynamics, since we do not have the enough technique to treat the non-perturbative effect.

The cancellation between the oblique corrections by assuming the contribution from the other additional new physics can be expected. Extended technicolor interaction, the additional broken gauge interactions inspired by GUTs, and so on can be the additional new physics. But the model will become very complicated and unnatural one.

For the completeness, the non-oblique correction should be considered in addition to the oblique correction. It is pointed out that the non-oblique correction due to the extended technicolor interaction is large, when the top quark is heavy 42. The nonoblique correction may cancel out the oblique correction. In next section we estimate the effect of the non-oblique correction in the extended technicolor theory.

\subsection{Technicolor and precision experiments II}

It is pointed out that the non-oblique correction to the $Z b \bar{b}$ vertex is generally large in the extended technicolor theory[42]. In this section, we explicitly calculate the non-oblique correction in a naive model of the extended technicolor theory. We show that the nonoblique correction is too large to be consistent with the experiments.

Many extended technicolor gauge bosons become massive, in the process of the breaking of the extended technicolor gauge symmetry. Some of them called sideways cause the transition of the ordinary fermions to the technifermions, some of them called horizontal connect the ordinary fermions themselves, and the others called "diagonal" diagonally interact with both the ordinary fermions and technifermions. The sideways bosons must exist in the realistic model to generate the quark and lepton masses, while the existence of the horizontal and the "diagonal" bosons is rather model-dependent. We consider the effect of the sideways and the "diagonal" from now on. The lightest sideways and "diagonal" gauge bosons are associated with the top quark. They make the largest contributions to the radiative corrections.

Consider four fundamental representations of the extended technicolor gauge group $S U\left(N_{T C}+1\right)$ containing the top and the bottom quark and the technifermions $U$ and $D$.

$$
\left.\left(\begin{array}{c}
U^{1} \\
\vdots \\
U^{N_{T C}} \\
t
\end{array}\right)_{L}\left(\begin{array}{c}
D^{1} \\
\vdots \\
D^{N_{T C}} \\
b
\end{array}\right)_{L}\right), \quad\left(\begin{array}{c}
U^{1} \\
\vdots \\
U^{N_{T C}} \\
t
\end{array}\right)_{R}, \quad\left(\begin{array}{c}
D^{1} \\
\vdots \\
D^{N_{T C}} \\
b
\end{array}\right)_{R} .
$$

Two left-handed representations form an $S U(2)_{L}$ doublet and two right-handed representations are $S U(2)_{L}$ singlets. (We assume that the extended technicolor gauge group commute with the weak interaction gauge group.) After the breaking of extended technicolor gauge group down to the technicolor gauge group $S U\left(N_{T C}\right)$, the massive sideways and "diagonal" gauge bosons are generated. 
Figure 5.10: The diagrams of the non-oblique correction to the $Z b \bar{b}$ vertex: (a) Sideways contribution. (b) "Diagonal" contribution.

In this model the mass of top quark is equal to the mass of bottom quark, because of the common mass and coupling of the sideways and $\langle\bar{U} U\rangle=\langle\bar{D} D\rangle$. In the realistic model, however, the right-handed top quark and the right-handed bottom quark must be contained in the different representations of the extended technicolor gauge group to realize the different sideways couplings. Therefore, the extended technicolor gauge theory must be a chiral gauge theory. However, realistic representations of extended technicolor gauge group are not known yet. Instead of considering explicit extended technicolor model, we here simply assume different extended technicolor couplings for top and bottom right-handed fundamental representations, while keeping the technicolor interaction vector-like. We also assume that $\langle\bar{U} U\rangle=\langle\bar{D} D\rangle$. This is a good toy model for the isospin breaking, although the extended technicolor gauge symmetry is destroyed. More explicitly, we set the sideways coupling $\xi_{t} g_{t}$ for the left-handed quarks, $g_{t} / \xi_{t}$ for the right-handed top quark, and $g_{t} / \xi_{b}$ for the right-handed bottom quark, where $g_{t}$ is given by

$$
m_{t} \simeq \frac{g_{t}^{2}}{M_{X}^{2}} 4 \pi F_{\pi}^{3}
$$

The scale $M_{X}$ is the mass of sideways and the relation $\langle\bar{U} U\rangle \simeq 4 \pi F_{\pi}^{3}$ (naive dimensional analysis) is used, where $F_{\pi}$ is the decay constant of the Nambu-Goldstone bosons of the technicolor chiral symmetry breaking. Large top quark mass indicates large value of $g_{t}$ or small value of $M_{X}$. We assume that the sideways effect can be treated perturbatively in loop calculation, namely, $\frac{\left(\xi_{t} g_{t}\right)^{2}}{4 \pi}<1$ and $\frac{\left(g_{t} / \xi_{t}\right)^{2}}{4 \pi}<1$. This relations restrict the value of $\xi_{t}$. For the realistic bottom quark mass, $\xi_{b}$ is restricted by $\frac{1}{\xi_{b}} \leq \frac{1}{\xi_{t}} \frac{m_{b}}{m_{t}}$.

The "diagonal" couplings are fixed through the relation to the sideways couplings. For technifermion, we obtain the "diagonal" coupling by multiplying the factor $-\frac{1}{N_{T C}} \sqrt{\frac{N_{T C}}{N_{T C}+1}}$ to their sideways couplings. For quark, we obtain it by multiplying the factor $\sqrt{\frac{N_{T C}}{N_{T C}+1}}$ to their sideways couplings. These factors come from the normalization and traceless property of the diagonal generator. The "diagonal" interaction is also chiral.

The sideways bosons yield potentially a large non-oblique correction to the $Z b \bar{b}$ vertex. By using the approach of the effective Lagrangian, the correction to the left-handed and the right-handed couplings of the bottom quark are derived as (Chivukula et al. in ref.42])

$$
\begin{aligned}
\delta g_{L}^{b} & =\frac{\xi_{t}^{2}}{4} \frac{m_{t}}{4 \pi F_{\pi}} \frac{e}{c s} \\
\delta g_{R}^{b} & =-\frac{1}{4 \xi_{b}^{2}} \frac{m_{t}}{4 \pi F_{\pi}} \frac{e}{c s}
\end{aligned}
$$

where $c$ and $s$ are the cosine and sine of the Weinberg angle, respectively. The suppression factor $m_{t} / 4 \pi F_{\pi}$ is not small for large $m_{t}$. The diagram corresponding to this correction is shown in fig.(5.10).

The "diagonal" boson " $X$ " also yields the non-oblique correction through the mixing 
Figure 5.11: The one-loop diagrams for calculating $X-W_{3}$ and $X-B$ mixing.

with $Z$ boson 43 . The mixing is parametrized by three parameters $x, y$, and $w$ as

$$
\begin{aligned}
\mathcal{L}_{A Z X}= & -\frac{1}{4}\left(\begin{array}{lll}
X_{\mu \nu} & Z_{\mu \nu} & A_{\mu \nu}
\end{array}\right)\left(\begin{array}{ccc}
1 & y & w \\
y & 1 & 0 \\
w & 0 & 1
\end{array}\right)\left(\begin{array}{c}
X^{\mu \nu} \\
Z^{\mu \nu} \\
A^{\mu \nu}
\end{array}\right) \\
& +\frac{1}{2}\left(\begin{array}{lll}
X_{\mu} & Z_{\mu} & A_{\mu}
\end{array}\right)\left(\begin{array}{ccc}
M_{X}^{2} & x M_{Z}^{2} & 0 \\
x M_{Z}^{2} & M_{Z}^{2} & 0 \\
0 & 0 & 0
\end{array}\right)\left(\begin{array}{c}
X^{\mu} \\
Z^{\mu} \\
A^{\mu}
\end{array}\right),
\end{aligned}
$$

where we set the "diagonal" mass equal to the sideways mass. Within the leading order of $x, y$, and $w$ in the four-fermion amplitude, the non-oblique correction to the $Z b \bar{b}$ vertex is obtained as

$$
\begin{aligned}
\delta g_{L}^{b} & =\xi_{t} g_{t} \sqrt{\frac{N_{T C}}{N_{T C}+1}} \frac{M_{Z}^{2}}{M_{X}^{2}-M_{Z}^{2}}(y-x), \\
\delta g_{R}^{b} & =\frac{g_{t}}{\xi_{b}} \sqrt{\frac{N_{T C}}{N_{T C}+1}} \frac{M_{Z}^{2}}{M_{X}^{2}-M_{Z}^{2}}(y-x) .
\end{aligned}
$$

We get $x, y$, and $w$ by calculating one-loop diagrams of fig.(5.11) with constant fermion mass. The results are

$$
\begin{aligned}
x= & N_{C} \sqrt{\frac{N_{T C}}{N_{T C}+1}} \frac{g_{t} e}{(4 \pi)^{2}} \frac{1}{c s}\left[\left(\xi_{t}-\frac{1}{\xi_{t}}\right) \frac{m_{U}^{2}-m_{t}^{2}}{M_{Z}^{2}}-\left(\xi_{t}-\frac{1}{\xi_{b}}\right) \frac{m_{D}^{2}-m_{b}^{2}}{M_{Z}^{2}}\right], \\
y= & -N_{C} \sqrt{\frac{N_{T C}}{N_{T C}+1}} \xi_{t} \frac{g_{t} e}{(4 \pi)^{2}} \frac{1}{c s} \frac{1}{3}\left[\ln \frac{m_{t}^{2}}{m_{b}^{2}}-\ln \frac{m_{U}^{2}}{m_{D}^{2}}\right] \\
& -N_{C} \sqrt{\frac{N_{T C}}{N_{T C}+1}} \frac{g_{t} e}{(4 \pi)^{2}} \frac{s}{c} \frac{2}{3}\left[\frac{2}{3}\left(\xi_{t}+\frac{1}{\xi_{t}}\right) \ln \frac{m_{U}^{2}}{m_{t}^{2}}-\frac{1}{3}\left(\xi_{t}+\frac{1}{\xi_{b}}\right) \ln \frac{m_{D}^{2}}{m_{b}^{2}}\right], \\
w= & N_{C} \sqrt{\frac{N_{T C}}{N_{T C}+1}} \frac{g_{t} e}{(4 \pi)^{2}} \frac{2}{3}\left[\frac{2}{3}\left(\xi_{t}+\frac{1}{\xi_{t}}\right) \ln \frac{m_{U}^{2}}{m_{t}^{2}}-\frac{1}{3}\left(\xi_{t}+\frac{1}{\xi_{b}}\right) \ln \frac{m_{D}^{2}}{m_{b}^{2}}\right] .
\end{aligned}
$$

Because the diagonal generator is traceless, the kinetic mixing parameters $y$ and $w$ are naturally finite. The mass mixing parameter $x$ should be naturally finite if we use the dynamical fermion mass having momentum dependence. To include the effect of dynamical mass, we set the momentum cutoff to the fermion mass in the individual loops, and get a finite $x$. The diagram corresponding to the correction of eq.(5.47) is shown in fig.(5.10).

The effect of technicolor resonances and Pseudo-Nambu-Goldstone bosons is not included in this one-loop calculation of $x, y$, and $w$. It can be expected that these effects do not change the sign and the order of magnitude of the correction unless we consider a special model. Actually in the estimation of the oblique correction due to the technicolor dynamics, the $S$ parameter estimated by the QCD scale up is just twice of the one 
Figure 5.12: The $\xi_{t}$ dependence of $\delta g_{L}^{b}$. The contributions of "diagonal" and Sideways boson are shown separately. Total contribution of extended technicolor bosons is also shown. The region of $\xi_{t}$ in which the perturbative loop calculation is valid is $0.7<\xi_{t}<1.4$.

Figure 5.13: Contours in $S-T$ plane (90\% C.L.). A contour with doted line is the result of two parameter $\left(S\right.$ and $T$ ) fitting. Another contour is a cross section (at $\left.\delta g_{L}^{b}=-0.0045\right)$ of the ellipsoid which is the result of three parameter fitting.

estimated by the one loop calculation, and the loop effect of pseudo-Nambu-Goldstone bosons is small without many light pseudo-Nambu-Goldstone bosons [37. Although these effects are important when we rigorously compare the theory with experiments, it can be expected that these effects are not important to show the importance of the non-oblique correction due to the extended technicolor gauge bosons. Therefore, we do not consider these effects in the estimation of $x, y$, and $w$.

The $\xi_{t}$ dependence of the both contributions to the left-handed coupling of the bottom quark are shown in fig.(5.12). We set $M_{X}=1 \mathrm{TeV}, F_{\pi}=125 \mathrm{GeV}$ (one family model), $m_{U}=m_{D}=\left(4 \pi F_{\pi}^{3}\right)^{1 / 3}, N_{T C}=4, m_{t}=150 \mathrm{GeV}$, and $M_{H}=1 \mathrm{TeV}$. The region of $\xi_{t}$ in which the perturbative loop calculation is reliable is $0.7<\xi_{t}<1$.4. The $\xi_{t}$ dependence of the sideways contribution is quadratic and strong. As for the "diagonal" contribution, it is approximately quadratic, but the dependence is very weak and the contribution is almost constant within the possible region of $\xi_{t}$. Both contributions are positive and do not cancel each other. The "diagonal" contribution is $30 \%$ of the sideways contribution when $\xi_{t}=1$. We can approximately write the "diagonal" contribution when $\xi_{t}=1$ as

$$
\delta g_{L}^{b}=\frac{1}{(4 \pi)^{4 / 3}} \frac{N_{C} N_{T C}}{N_{T C}+1} \frac{m_{t}}{4 \pi F_{\pi}} \frac{e}{c s}
$$

which should be compared with eq.(5.45) with $\xi_{t}=1$.

Both the sideways and the "diagonal" contributions to the right-handed $Z b \bar{b}$ coupling, eqs. (5.45) and (5.47) are suppressed at least by the power of $\frac{1}{\xi_{b} \xi_{t}}$. Namely, these contributions are suppressed by the power of $\frac{m_{b}}{m_{t}} \frac{1}{\xi_{t}^{2}}$ compared with the contributions to the left-handed coupling. Therefore, the contributions to the right-handed coupling are not important unless the predictions are highly sensitive to the change of right-handed coupling. We ignore it from now on.

Both sideways and "diagonal" bosons also contribute to the oblique correction, $S, T$ and $U$. However, since the contribution is the two-loop effect, it is small in comparison with the contribution due to the technicolor dynamics. For example, the "diagonal" contribution to the $S$ parameter is $S=0.022$ for $M_{X}=1 \mathrm{TeV}, F_{\pi}=125 \mathrm{GeV}, N_{T C}=$ $4, \xi_{t}=1, m_{t}=150 \mathrm{GeV}$, and $M_{H}=1 \mathrm{TeV}$, which is small in comparison with the contribution $S>0.4$ due to the technicolor dynamics.

The non-oblique corrections eqs.(5.45) and (5.47) change the predictions. For example, the total $Z$ width can be written as

$$
\Gamma_{Z}=\Gamma_{Z}^{S M}+\delta \Gamma_{Z}+a S+b T
$$


Figure 5.14: Cross sections of the ellipsoid of 90\% C.L. at $\delta g_{L}^{b}=0,0.003,0.006$.

Figure 5.15: Cross sections of the ellipsoid of $90 \%$ C.L. at $T=-0.11$ (best fit), 0.5, 1.0. Horizontal line means the lower bound of the orediction of the extended technicolor thoery.

where $\Gamma_{Z}^{S M}$ is the one-loop prediction of the standard model and

$$
\delta \Gamma_{Z}=\frac{\partial \Gamma_{Z}^{S M}}{\partial g_{L}^{b}} \delta g_{L}^{b} .
$$

The coefficients $a$ and $b$ are written by the standard model parameters as

$$
\begin{gathered}
a=-\frac{\alpha^{2} M_{Z}}{12 s^{2} c^{2}\left(c^{2}-s^{2}\right)} \sum_{f}\left(I_{3 f}-s^{2} Q_{f}\right) Q_{f} N_{f}, \\
b=\frac{\alpha^{2} M_{Z}}{6 s^{2} c^{2}} \sum_{f}\left\{\left(I_{3 f}-s^{2} Q_{f}\right)^{2} N_{f}+\frac{2 s^{2} c^{2}}{c^{2}-s^{2}}\left(I_{3 f}-s^{2} Q_{f}\right) Q_{f} N_{f}\right\},
\end{gathered}
$$

where $I_{3 f}, Q_{f}$, and $N_{f}$ denote weak isospin, electric charge, and effective number of color with respect to the fermion of flavor $f$, respectively [37]. The predictions for the $\mathrm{R}$ ratio on $Z$ pole and forward-backward asymmetry of $b$ quark are also changed in the same way as $\Gamma_{Z}$. We analyze the experiments by using three free parameters $S, T, \delta g_{L}^{b}$ and get an ellipsoid in $S-T-\delta g_{L}^{b}$ space. The region inside the ellipsoid is favored by the experiments. We do not consider the experiments which are parametrized by $U$ parameter, for simplicity.

The cross section of the ellipsoid of $90 \%$ C.L., which contains a best-fit point, is shown in fig.(5.13). The following experiments are considered; total $Z$ width, $R$ ratio on $Z$ pole, forward-backward asymmetry of $b$ and $\mu$, polarization asymmetry of $\tau$, deep inelastic neutrino scattering $\left(g_{L}\right.$ and $\left.g_{R}\right)$, and atomic parity violation $Q_{W}\left({ }_{53}^{133} C_{S}\right)$ [40]. The most favorable values are $\delta g_{L}^{b}=-0.0045, S=-0.73$, and $T=-0.11$. The favorable region in $S-T$ plane becomes larger than the one from two parameter analysis. Fig.(5.14) shows the favorable regions in $S-T$ plane $\left(90 \%\right.$ C.L.) when $\delta g_{L}^{b}$ is fixed in several values. We find that the new physics contribution to the left-handed coupling of $b$ quark must be smaller than 0.007 in $90 \%$ C.L. This value is much smaller than the extended technicolor contributions which is shown in fig.(5.12).

Fig.(5.15) shows the cross sections of ellipsoid at several values of $T$. We also see that the extended technicolor contributions are at the outside of the ellipsoid of 90\% C.L.

We showed that the non-oblique corrections to $Z b \bar{b}$ vertex which are naturally expected to exist in the realistic extended technicolor model are too large to be consistent with the experiments. The reason is that only the predictions to the total $\mathrm{Z}$ width, the $\mathrm{R}$ ratio on $\mathrm{Z}$ pole, and the forward-backward asymmetry of the $\mathrm{b}$ quark which relate with the $Z b \bar{b}$ vertex are modified. There must be some mechanisms which reduce or cancel this non-oblique correction in order that the extended technicolor model is consistent with the experiments. 
In the phenomenological test of the extended technicolor models we must consider both non-oblique and oblique corrections. The physics which simultaneously reduce both oblique and non-oblique corrections is expected in model building of the extended technicolor theory. 


\section{Chapter 6}

\section{Tumbling gauge theory}

Tumbling gauge theory [9] contains the many hierarchical scales. No elementary scalar fields are needed to realize the hierarchical gauge symmetry breaking. Many effective four fermion interactions with the hierarchical scales are generated in the process of "tumbling". Therefore, the theory has been considered as the candidate of the dynamics of the extended technicolor theory. Tumbling gauge theory is the special kind of the chiral gauge theory. It is also a good property to be the dynamics of the extended technicolor theory, since the extended technicolor theory should be the chiral gauge theory for the realistic fermion masses, as we have already explained in chapter 4 . In this chapter, we formulate the tumbling gauge theory by using the Cornwall-Jackiw-Tomboulis (CJT) effective action [17, which have already introduced in section 3.3.

The chiral gauge theory is the gauge theory with the fermions which representation is not real as a whole. As an example of the chiral gauge theory, consider the one generation of the standard model.

$$
\begin{aligned}
\left(\begin{array}{c}
u \\
d
\end{array}\right)_{L} & \sim(3,2,1 / 6), \\
\left(u_{R}\right)^{c} & \sim\left(3^{*}, 1,-2 / 3\right), \\
\left(d_{R}\right)^{c} & \sim\left(3^{*}, 1,1 / 3\right), \\
\left(\begin{array}{c}
\nu_{e} \\
e
\end{array}\right)_{L} & \sim(1,2,-1 / 2), \\
\left(e_{R}\right)^{c} & \sim(1,1,1),
\end{aligned}
$$

where $(3,2,1 / 6)$, for instance, means the $S U(3)_{c}$ triplet, the $S U(2)_{L}$ doublet, and $Y / 2=$ $1 / 6\left(Y / 2\right.$ is the charge of $\left.U(1)_{Y}\right)$. All the fermion fields are written as the left-handed chiral fields. If we take the complex conjugate of this representation, we get

$$
\begin{aligned}
\left(\begin{array}{c}
\tilde{u} \\
\tilde{d}
\end{array}\right)_{L} & \sim\left(3^{*}, 2,-1 / 6\right), \\
\left(\tilde{u}_{R}\right)^{c} & \sim(3,1,2 / 3), \\
\left(\tilde{d}_{R}\right)^{c} & \sim(3,1,-1 / 3),
\end{aligned}
$$

\footnotetext{
${ }^{1}$ Here, we do not consider the elementary scalar fields.
} 


$$
\begin{aligned}
\left(\begin{array}{c}
\tilde{\nu}_{e} \\
\tilde{e}
\end{array}\right)_{L} & \sim(1,2,1 / 2), \\
\left(\tilde{e}_{R}\right)^{c} & \sim(1,1,-1) .
\end{aligned}
$$

(Remember that all the representation of the $S U(2)$ group is real.) If we consider only the $S U(3)$ gauge group, this representation is equivalent to the original one as a whole There are same number of 3 and $3^{*}$. We call this representation real or vector-like one with respect to $S U(3)_{c}$. But, the conjugated representation are not equivalent to the original one when we consider the $S U(2)_{L} \times(1)_{Y}$ gauge group. We call such representation chiral representation. The standard model is a chiral gauge theory.

In the chiral gauge theory, the cancellation of the gauge anomaly is non-trivial. It must be canceled so that the theory is renormalizable. We must impose the condition

$$
\operatorname{tr}\left(T^{a}\left\{T^{b}, T^{c}\right\}\right)=0
$$

for any combination of the generators of the gauge symmetry, $T^{a}, T^{b}$, and $T^{c}$, where the generators are represented as the large matrixes which act on the space of the whole fermion contents. It is famous that the non-trivial cancellation is accomplished in the standard model.

The tumbling gauge theory can be formulated by using the CJT effective action. Let us consider the non-Abelian gauge group $G$ and left-handed fermions in the irreducible representation $r_{1}, r_{2}, \cdots$. We consider the chiral representation and impose the cancellation of the gauge anomaly. And we consider the situation that the fermion contents are compatible with asymptotic freedom. In order to know the remaining symmetry at the low energy and the symmetry breaking scales, we must solve the dynamics of this theory. We consider the CJT effective action which includes only one gauge boson exchange vacuum diagram, and introduce the most attractive channel (MAC) hypothesis 9.

For simplicity, we assume that $G$ is a simple group. It is convenient to represent all the left-handed fermions as a Majorana fermion field

$$
\Psi \equiv\left(\begin{array}{c}
\psi_{L}\left(r_{1}\right) \\
\psi_{L}\left(r_{2}\right) \\
\vdots
\end{array}\right)+\left(\begin{array}{c}
\psi_{L}\left(r_{1}\right)^{c} \\
\psi_{L}\left(r_{2}\right)^{c} \\
\vdots
\end{array}\right)
$$

Note that the generators of $G$ acting on this field are defined as

$$
\mathcal{T}^{a} \equiv T^{a}\left(\frac{1-\gamma_{5}}{2}\right)-T^{a *}\left(\frac{1+\gamma_{5}}{2}\right)
$$

where $T^{a}$ denotes the generator which acts on the space of the whole fermion contents. Then the effective action can be written as

$$
\Gamma[S]=\frac{1}{2} \ln \operatorname{Det}\left(S^{-1}\right)-\frac{1}{2} \operatorname{Tr}(\not p S)+D[S],
$$

where $S$ denotes the full propagator of the Majorana field $\Psi$ and $D[S]$ is the set of all two-particle irreducible (2PI) vacuum diagrams (fig.(6.1)). The full propagator $S$ contains 
Figure 6.1: Two particle irreducible vacuum diagrams in effective action.

the mass function $\Sigma$ as

$$
S^{-1}(p)=\Sigma(p)-\not p
$$

When we consider the one gauge boson exchange diagram in fig.(6.1) only, we get the Schwinger-Dyson equation (gap equation) in ladder approximation

$$
\Sigma(p)=-g^{2} \int \frac{d^{4} k}{(2 \pi)^{4} i} \gamma_{\nu} \mathcal{T}^{a} S(k) \gamma_{\mu} \mathcal{T}^{a} D^{\mu \nu}(k-p)
$$

as a stationary condition of this effective action (See section 3.3), where $g$ is the gauge coupling constant and $D_{\mu \nu}$ is the free gauge boson propagator. We take Landau gauge so that the ladder approximation is consistent with the Ward-Takahashi identity (See section 3.1.). In Landau gauge, $\Sigma(p)$ does not contain the $\gamma$-matrix, and it becomes the function of $p^{2}$. The mass function $\Sigma\left(p^{2}\right)$ can be decomposed as

$$
\Sigma\left(p^{2}\right)=\Sigma\left(p^{2}\right)_{r_{1} \times r_{2} \rightarrow r_{c}^{12}} \oplus \Sigma\left(p^{2}\right)_{r_{1} \times r_{3} \rightarrow r_{c}^{13}} \oplus \cdots
$$

corresponding to the channel of the pair condensation $r_{1} \times r_{2} \rightarrow r_{c}^{12}, r_{1} \times r_{3} \rightarrow r_{c}^{13}, \cdots$, where $r_{c}$ 's denote the representation of the condensates.

It is enough to consider the linearized gap equation to estimate the scale of the condensate. By linearizing the equation with respect to the mass function, each condensation channels can be treated separately 2 , since we can decompose the mass function as eq. 6.7). We concentrate on the channel $r_{1} \times r_{2} \rightarrow r_{c}$ from now on. We get the linearized gap equation of the channel

$$
\begin{gathered}
\Sigma_{A}\left(-p^{2}\right)=\lambda\left(r_{1}, r_{2}, r_{c}\right) \int d k^{2} \frac{\Sigma_{A}\left(-k^{2}\right)}{k^{2}+m^{2}}\left\{\theta\left(k^{2}-p^{2}\right)+\frac{k^{2}}{p^{2}} \theta\left(p^{2}-k^{2}\right)\right\}, \\
\lambda\left(r_{1}, r_{2}, r_{c}\right) \equiv \frac{3}{4 \pi} \cdot \frac{g^{2}}{4 \pi} \cdot \frac{1}{2}\left\{C_{2}\left(r_{1}\right)+C_{2}\left(r_{2}\right)-C_{2}\left(r_{c}\right)\right\}
\end{gathered}
$$

where $A$ is the index of the representation $r_{c}, C_{2}(r)$ is the coefficient of the second Casimir operator of representation $r$, and $m=\Sigma_{A}\left(m^{2}\right)$. The integration is performing in Euclidean space and the angular integration has already done. The effective coupling of the channel $r_{1} \times r_{2} \rightarrow r_{c}$ is defined by $\lambda\left(r_{1}, r_{2}, r_{c}\right)$. When the gauge group $G$ is not a simple group but a direct product group, the effective coupling becomes the sum of the effective couplings with respect to each gauge interactions. We call the channel with the largest effective coupling most attractive channel (MAC). The above gap equation has a non-trivial solution when $\lambda$ exceeds the critical value $\lambda_{c}=1 / 4$, as we estimated in section 3.1.

We can obtain hierarchical condensation scales in the MAC hypothesis. The quantities in the (exact) effective action, the gauge coupling $g$, for instance, are renormalized quantities with scale dependence. Here we approximately replace the gauge coupling constant $g$ by the running coupling $g(\mu)$ of the one-loop perturbative calculation, where $\mu$ is

\footnotetext{
${ }^{2}$ We get the independent equations for each channels. There is not the mixing of the channels.
} 
a reference of the energy scale. As the running coupling becomes large in the low energy region, the effective coupling of the MAC reaches "firstly" the critical value $\lambda_{c}=1 / 4$ at a certain scale $M$ defined by

$$
\lambda(M)=\frac{3}{4 \pi} \cdot \frac{g^{2}(M)}{4 \pi} \cdot \frac{1}{2}\left\{C_{2}\left(r_{1}\right)+C_{2}\left(r_{2}\right)-C_{2}\left(r_{c}\right)\right\}=\frac{1}{4} .
$$

Then the condensate of this channel is likely to emerge or become non-negligible in comparison with the reference of the energy scale $M$. We here assume the MAC hypothesis that the condensate is formed in not all the attractive channels but only in the MAC. Since the MAC condensation is gauge non-singlet in general, the symmetry breaking takes place not only on the global symmetry but also on the gauge symmetry itself. Some gauge bosons and fermions get their masses of the order of $M$ by this symmetry breaking. The physics below the scale $M$ can be described by an effective theory containing gauge bosons of reduced gauge symmetry and massless fermions.

In the original tumbling scenario, the particles which get masses of order $M$ are neglected and it is naively assumed that they decouple from the low energy dynamics. In the effective theory the scale $M$ has a meaning of the ultraviolet momentum cutoff. The values of the running gauge couplings in the theory above and below the scale $M$ must coincide with each other at the scale $M$ because of the continuity of the theory and the uniqueness of the fundamental scale of the theory. We can apply the above effective action argument to the effective theory and find the MAC and the next condensation scale $M^{\prime}$. The effective theory below the scale $M^{\prime}$ has a further reduced gauge symmetry. In this way, the gauge symmetry can be broken one after another by itself and the scale hierarchy $M, M^{\prime}, \cdots$ can be generated ("tumbling"). The sequence of the gauge symmetry breaking continues until the representation of the massless fermions becomes real as a whole, provided that the asymptotic freedom of the gauge coupling holds. The sequence of the condensation scales (not always taking with the gauge symmetry breaking) terminates when all the fermions with attractive gauge interaction become massive.

As an example, consider the gauge group $S U(5)$ and the chiral representations $5^{*}$ and 10 which can be written as

$$
\Psi_{5^{*}}=\left(\begin{array}{c}
\psi_{5^{*}}^{1} \\
\psi_{5^{*}}^{2} \\
\psi_{5^{*}}^{3} \\
\psi_{5^{*}}^{4} \\
\psi_{5^{*}}^{5}
\end{array}\right), \quad \Psi_{10}=\left(\begin{array}{ccccc}
0 & \psi^{12} & \psi^{13} & \psi^{14} & \psi^{15} \\
-\psi^{12} & 0 & \psi^{23} & \psi^{24} & \psi^{25} \\
-\psi^{13} & -\psi^{23} & 0 & \psi^{34} & \psi^{35} \\
-\psi^{14} & -\psi^{24} & -\psi^{34} & 0 & \psi^{45} \\
-\psi^{15} & -\psi^{25} & -\psi^{35} & -\psi^{45} & 0
\end{array}\right) .
$$

These fermions are the left-handed fermions. This representation is the same as the one generation in the $S U(5) \mathrm{GUT}$ 44]. The gauge anomaly is canceled out, and the theory is asymptotically free. The following channel of the pair condensation can be considered.

$$
\begin{aligned}
10 \times 10 & =5^{*}+45+50, \\
5^{*} \times 5^{*} & =10^{*}+15^{*}, \\
5^{*} \times 10 & =5+45^{*} .
\end{aligned}
$$

Since there is no singlet in the right-hand side (representation of the condensate), the pair condensates should break the gauge symmetry. The coefficients of the effective couplings 
of each channels $\left\{C_{2}\left(r_{1}\right)+C_{2}\left(r_{2}\right)-C_{2}\left(r_{c}\right)\right\}$ are as follows.

$$
\begin{aligned}
10 \times 10 & \rightarrow\left\{\begin{array}{lr}
5^{*} & \frac{24}{5} \\
45 & \frac{4}{5} \\
50 & -\frac{6}{5}
\end{array}\right. \\
5^{*} \times 5^{*} & \rightarrow\left\{\begin{array}{cr}
10^{*} & \frac{6}{5} \\
15^{*} & -\frac{4}{5}
\end{array}\right. \\
5^{*} \times 10 & \rightarrow\left\{\begin{array}{cc}
5 & \frac{18}{5} \\
45^{*} & -\frac{2}{5}
\end{array}\right.
\end{aligned}
$$

The MAC is the channel $10 \times 10 \rightarrow 5^{*}$. The condensation of the representation $5^{*}$ breaks the $S U(5)$ gauge symmetry to the $S U(4)$ gauge symmetry.

The fermion contents after the breaking is

$$
\begin{gathered}
\Psi_{4^{*}}=\left(\begin{array}{c}
\psi_{5^{*}}^{1} \\
\psi_{5^{*}}^{2} \\
\psi_{5^{*}}^{3} \\
\psi_{5^{*}}^{4}
\end{array}\right), \quad \Psi_{1}=\psi_{5^{*}}^{5}, \\
\Psi_{6}=\left(\begin{array}{cccc}
0 & \psi^{12} & \psi^{13} & \psi^{14} \\
-\psi^{12} & 0 & \psi^{23} & \psi^{24} \\
-\psi^{13} & -\psi^{23} & 0 & \psi^{34} \\
-\psi^{14} & -\psi^{24} & -\psi^{34} & 0
\end{array}\right), \quad \Psi_{4}=\left(\begin{array}{c}
\psi^{15} \\
\psi^{25} \\
\psi^{35} \\
\psi^{45}
\end{array}\right) .
\end{gathered}
$$

The condensed fermion is $\Psi_{6}$, and it has the Majorana mass of the order of the $S U(5)$ breaking scale. Other fermions remain massless at this stage.

The $S U(4)$ gauge coupling becomes large at low energy, and the subsequent pair condensation occurs. The MAC is the singlet channel $4 \times 4^{*} \rightarrow 1$, and the $S U(4)$ gauge symmetry is not broken. The two fermions 4 and $4^{*}$ form a Dirac massive fermion. All the fermions except for the singlet $\Psi_{1}$ become massive. No subsequent condensation occurs.

The above estimation is not the rigorous one, since there are some assumptions: the ladder approximation, the MAC hypothesis, and neglecting the massive particles in the effective theory. Moreover, we did not specify the regularization scheme and the renormalization. In fact, the explicit momentum cut off regularization in the above argument is dangerous, since it breaks the chiral gauge symmetry f?. More rigorous treatment is needed to apply this dynamics to the physics. In this thesis, we believe that the result is at least qualitatively good, while it may not be quantitatively good.

It is interesting whether the gauge group of the standard model can be included or not in the low energy effective theory of the tumbling gauge theories. It is systematically estimated for the tumbling gauge theories with simple gauge group $S U(N)$ [46]. Assuming the simple group means to consider the grand unified theory with the extended technicolor. The restrictions of the asymptotic freedom and the gauge anomaly cancellation reduces

\footnotetext{
${ }^{3}$ We have not known the regularization scheme which preserve the chiral gauge symmetry. But, recently a method which use the infinite number of the Pauli-Villars fields is proposed 45.]. We hope that the regularization method proceeds our understanding on the chiral gauge theory. Especially, we hope the lattice calculation.
} 
the number of the models which should be considered. The result is the discouraging one. There are no candidate which includes the gauge group of the standard model in the low energy effective theory. If we replace the MAC hypothesis into more rigorous one, we may have another pattern of the tumbling and may get the candidates.

In the next chapter, we consider the tumbling including the massive particles which get their masses in the process of tumbling. After the second stage of the tumbling, there exist massive fermions and the effective four fermion interactions which are mediated by the massive gauge bosons. 


\section{Chapter 7}

\section{Tumbling and technicolor}

As we explained in chapter 5, the technicolor dynamics should be accompanied with the large anomalous dimension to avoid the flavor-changing neutral current problem. Two explicit dynamical models with large anomalous dimension are proposed. One is the gauge theory with very slowly running coupling (walking dynamics) [11], and another is the gauge theory with strong four fermion interaction (gauged Nambu-Jona-Lasinio model) [12].

It is also explained that the dynamics of the tumbling gauge theory is a candidate of the dynamics of the extended technicolor theory. The effective four fermion interactions with hierarchical scales can be translated to the hierarchy of the masses of the ordinary fermions. Moreover, if the four fermion interactions between the technifermions can be strong, the large anomalous dimension is realized. In the following, we estimate the strength of the effective four fermion interactions in the tumbling gauge theory, and get the simple formula to give the values of the coupling constants of the interactions. We also discuss of the decoupling of the massive gauge bosons which get their masses in the process of tumbling from the low energy dynamics. The decoupling of the massive fermions which get their masses in the process of tumbling is also discussed. The decoupling of the massive particles has already been proved in the perturbative point of view 47]. But it is still non-trivial in the non-perturbative point of view.

\subsection{The massive gauge boson effect}

In the process of tumbling, gauge bosons associated with the broken generators become massive. In the ordinary scenario, these massive gauge bosons are assumed to decouple from the low energy dynamics, and they are only perturbatively considered as the weak effective four fermion interactions in the low energy region. However, if the four fermion interaction can be strong, their non-perturbative effect must be considered.

Consider a certain stage of tumbling such that the gauge group $G$ breaks to $H$ at the scale $M$. For simplicity, we regard the groups $G$ and $H$ as the simple ones. Extension to the direct product groups is straightforward and we will discuss it later. The gauge bosons

with respect to the generators of $G / H$ become massive in this process. The representation of the fermions are denoted by $r_{1}^{G}, r_{2}^{G}, \cdots$ in the theory above the scale $M$, and by $r_{1}^{H}$, $r_{2}^{H}, \cdots$ in the theory below the scale $M$. 
The effective action below the scale $M$ is given by decomposing the effective action at the scale $M$. Above the scale $M$, the effective action with respect to the channel $r_{1}^{G} \times r_{2}^{G} \rightarrow r_{c}^{G}$ is

$$
\Gamma[\Sigma]=H\left[\Sigma^{* A} \Sigma_{A}\right]+\frac{g_{G}^{2}}{2}\left\{C_{2}\left(r_{1}^{G}\right)+C_{2}\left(r_{2}^{G}\right)-C_{2}\left(r_{c}^{G}\right)\right\} F\left[\Sigma^{* A} \Sigma_{A}\right],
$$

where the functional

$$
H\left[\Sigma^{* A} \Sigma_{A}\right] \equiv-2 \int \frac{d^{4} p}{(2 \pi)^{4}} \frac{1}{p^{2}-m^{2}} \Sigma^{* A}\left(p^{2}\right) \Sigma_{A}\left(p^{2}\right)
$$

comes from the fermion integration, the functional

$$
\begin{aligned}
& F\left[\Sigma^{* A} \Sigma_{A}\right] \equiv \\
& \quad-2 i \int \frac{d^{4} p}{(2 \pi)^{4} i} \frac{d^{4} k}{(2 \pi)^{4} i} \frac{1}{(k+p)^{2}-m^{2}} \frac{1}{p^{2}-m^{2}} \cdot D_{\mu}^{\mu}(k) \Sigma^{* A}\left((k+p)^{2}\right) \Sigma_{A}\left(p^{2}\right)
\end{aligned}
$$

comes from one gauge boson exchange vacuum diagram, $g_{G}$ is the gauge coupling constant in the theory above the scale $M$, and $A$ is the group index of the condensate of representation $r_{c}^{G}$ composed of $r_{1}^{G}$ and $r_{2}^{G}$ 円. This effective action has already been linearized with respect to $\Sigma^{* A} \Sigma_{A}$, therefore we get the linearized gap equation in ladder approximation directly by differentiating with respect to $\Sigma^{* A}$. We are assuming that the condensation in MAC emerges at the scale $M$ and gauge group $G$ is broken to $H$, according to the formulation which is explained in the previous chapter.

The mass function $\Sigma_{A}\left(p^{2}\right)$ is decomposed into the irreducible components of the group $H, r_{c 1}^{H}, r_{c 2}^{H}, \ldots r_{c n}^{H}$, as

$$
\Sigma_{A}\left(p^{2}\right)=\Sigma_{i_{1}}^{\left(r_{c 1}^{H}\right)}\left(p^{2}\right) \oplus \Sigma_{i_{2}}^{\left(r_{c 2}^{H}\right)}\left(p^{2}\right) \oplus \ldots \oplus \Sigma_{i_{n}}^{\left(r_{c n}^{H}\right)}\left(p^{2}\right),
$$

where $i_{k}$ is the group index of the representation $r_{c k}^{H}$. The fermion representation can also be decomposed as

$$
\begin{aligned}
r_{1}^{G} & =r_{11}^{H} \oplus r_{12}^{H} \oplus \ldots \oplus r_{11_{1}}^{H}, \\
r_{2}^{G} & =r_{21}^{H} \oplus r_{22}^{H} \oplus \ldots \oplus r_{2 n_{2}}^{H}, \\
& \vdots
\end{aligned}
$$

The decomposed mass functions correspond to all the possible condensates composed of $r_{11}^{H}, r_{12}^{H}, \cdots, r_{1 n_{1}}^{H}$ and $r_{21}^{H}, r_{22}^{H}, \cdots, r_{2 n_{2}}^{H}$. But the correspondence is not one to one. For example, if both pairs, $r_{11}^{H} \times r_{21}^{H}$ and $r_{12}^{H} \times r_{22}^{H}$, contain the same representation $r_{c 1}^{H}$, the mass function $\Sigma_{i_{1}}^{\left(r_{c 1}^{H}\right)}$ is the sum of two independent mass functions of the two channels with certain weight. This means the mixing of the two channels of $r_{11}^{H} \times r_{21}^{H} \rightarrow r_{c 1}^{H}$ and $r_{12}^{H} \times r_{22}^{H} \rightarrow r_{c 1}^{H}$. Except for these mixings, the correspondence is one to one. We ignore the mixings for simplicity from now on.

\footnotetext{
${ }^{1}$ Since we take the Landau gauge, the mass function is the function of $p^{2}$ instead of $p$.

${ }^{2}$ The mixing is caused by the exchange of the massive gauge bosons corresponding to the broken off-diagonal generators which cause the transition $r_{11}^{H} \longrightarrow r_{12}^{H}$ and $r_{22}^{H} \longrightarrow r_{21}^{H}$, for example. The other contributions are diagonal.
} 
Accordingly, the effective action is decomposed as

$$
\begin{aligned}
\Gamma[\Sigma] & =H\left[\Sigma^{* i_{1}\left(r_{c 1}^{H}\right)} \sum_{i_{1}}^{\left(r_{c 1}^{H}\right)}\right]+\frac{g_{G}^{2}}{2}\left\{C_{2}\left(r_{1}^{G}\right)+C_{2}\left(r_{2}^{G}\right)-C_{2}\left(r_{c}^{G}\right)\right\} F\left[\Sigma^{* i_{1}\left(r_{c 1}^{H}\right)} \sum_{i_{1}}^{\left(r_{c 1}^{H}\right)}\right] \\
& +\left(r_{c 1}^{H} \longrightarrow r_{c 2}^{H}, r_{c 3}^{H}, \cdots\right) .
\end{aligned}
$$

There are no cross terms of the decomposed mass functions of the different representations. We concentrate on a channel $r_{11}^{H} \times r_{21}^{H} \rightarrow r_{c 1}^{H}$ for a while. Because the functional $F$ linearly contains a gauge boson propagator, it can be decomposed into two parts concerning $H$ and $G / H$, respectively as

$$
\begin{aligned}
& \frac{g^{2}}{2}\left\{C_{2}\left(r_{1}^{G}\right)+C_{2}\left(r_{2}^{G}\right)-C_{2}\left(r_{c}^{G}\right)\right\} F\left[\Sigma^{* i_{1}\left(r_{c 1}^{H}\right)} \sum_{i_{1}}^{\left(r_{c 1}^{H}\right)}\right] \\
& \quad=a_{H} F\left[\Sigma^{* i_{1}\left(r_{c 1}^{H}\right)} \sum_{i_{1}}^{\left(r_{c 1}^{H}\right)}\right]+a_{G / H} F\left[\Sigma^{* i_{1}\left(r_{c 1}^{H}\right)} \sum_{i_{1}}^{\left(r_{1}^{H}\right)}\right],
\end{aligned}
$$

where $a_{H}$ and $a_{G / H}$ are constants satisfying

$$
a_{H}+a_{G / H}=\frac{g_{G}^{2}}{2}\left\{C_{2}\left(r_{1}^{G}\right)+C_{2}\left(r_{2}^{G}\right)-C_{2}\left(r_{c}^{G}\right)\right\} .
$$

The contribution of the unbroken gauge interaction is known as

$$
a_{H}=\frac{g_{H}^{2}}{2}\left\{C_{2}\left(r_{11}^{H}\right)+C_{2}\left(r_{21}^{H}\right)-C_{2}\left(r_{c 1}^{H}\right)\right\},
$$

where $g_{H}$ is the gauge coupling in the theory below the scale $M$. As a matching condition we have $g_{H}(M)=g_{G}(M)$. The above decomposition has to be done at the scale $M$, then we obtain the value of $a_{G / H}$ as

$$
a_{G / H}=\frac{g_{G}^{2}(M)}{2}\left\{C_{2}\left(r_{1}^{G}\right)+C_{2}\left(r_{2}^{G}\right)-C_{2}\left(r_{c}^{G}\right)\right\}-\frac{g_{H}^{2}(M)}{2}\left\{C_{2}\left(r_{11}^{H}\right)+C_{2}\left(r_{21}^{H}\right)-C_{2}\left(r_{c 1}^{H}\right)\right\} .
$$

We can get the effective action below the scale $M$ by giving masses of the order of $M$ to the gauge bosons $G / H$ and the fermions which would become massive in this process.

We can relate $a_{G / H}$ to the coupling constant of the effective four fermion interaction. The gap equation with respect to the channel $r_{11}^{H} \times r_{21}^{H} \rightarrow r_{c 1}^{H}$ is given by differentiating the effective action with respect to $\Sigma_{c 1}^{\left(r_{c 1}^{H}\right) *}$ as

$$
\begin{aligned}
\Sigma_{i_{1}}^{\left(r_{1}^{H}\right)}\left(-q^{2}\right) & =\lambda^{\left(r_{c 1}^{H}\right)} \int^{M^{2}} d p^{2} \frac{\Sigma_{i_{1}}^{\left(r_{c 1}^{H}\right)}\left(-p^{2}\right)}{p^{2}+m^{2}}\left\{\theta\left(q^{2}-p^{2}\right) \frac{p^{2}}{q^{2}}+\theta\left(p^{2}-q^{2}\right)\right\} \\
& +\frac{g_{4 F}^{\left(r_{c 1}^{H}\right)}}{M^{2}} \int^{M^{2}} d p^{2} \Sigma_{i_{1}}^{\left(r_{c 1}^{H}\right)}\left(-p^{2}\right),
\end{aligned}
$$

where we approximate the massive gauge boson propagator as $g_{\mu \nu} / M^{2}$ and the integration is performed in Euclidean space. Here we ignore the massive fermions which get their masses of the order of $M$ at the breaking of $G \rightarrow H$. The effect of the massive fermions will 
Figure 7.1: Phase diagram of gauged Nambu-Jona-Lasinio model in ladder and fixed coupling approximation. The region inside the critical line is symmetric phase and outside is broken phase. The anomalous dimension of the mass function is given by $\gamma_{m}=1+$ $\left(1-\lambda / \lambda_{c}\right)^{1 / 2}$. It increases from $\gamma_{m}=1$ to $\gamma_{m}=2$ along the critical line.

be discussed in the next section. This is the linearized gap equation of the gauged NambuJona-Lasinio model in the ladder approximation and we defined the effective couplings of gauge and four fermion interaction as

$$
\lambda^{\left(r_{c 1}^{H}\right)}=\frac{3}{4 \pi} \cdot \frac{a_{H}}{4 \pi}
$$

and

$$
g_{4 F}^{\left(r_{c 1}^{H}\right)}=\frac{3}{4 \pi} \cdot \frac{a_{G / H}}{4 \pi},
$$

respectively. This four fermion coupling measures the size of the contribution of the massive gauge boson to the dynamics of this channel.

The solution of this gap equation and phase diagram (fig.(7.1)) are given in ref. 48. The critical line can be obtained as follows. The eq.(7.12) with $m=0$ (expanding the Schwinger-Dyson equation around the zero of the mass function) can be transformed to the differential equation and boundary conditions

$$
\begin{gathered}
(x \Sigma(x))^{\prime \prime}+\lambda \frac{\Sigma(x)}{x}=0, \\
\lim _{x \rightarrow \mu^{2}} x^{2} \Sigma^{\prime}(x)=0, \\
\lim _{x \rightarrow M^{2}}\left[(x \Sigma(x))^{\prime}+\frac{g_{4 F}}{\lambda} x \Sigma(x)^{\prime}\right]=0,
\end{gathered}
$$

where $x$ is the squared Euclidean momentum: $x=q^{2}$ (We write $\Sigma\left(-q^{2}\right)$ as $\Sigma(x)$.), and $\mu$ is the scale which will be set to zero in the following. We neglect the group indexes for simplicity. The effect of the four fermion interaction comes in only through the ultraviolet boundary condition (see eqs.(3.49), (3.50), and (3.51).). Although the bifurcation theory 18] can not be used to obtain the critical line, because we have two parameters $g_{4 F}$ and $\lambda$, it can be obtained as a condition to exist the non-trivial solution. It is enough to consider the above linearized equation, if we consider only the small solution. While both couplings are small, there is only a trivial solution. And when the couplings become large and satisfy a certain relation, the small non-trivial solution will be bifurcated from the trivial solution. The relation is nothing but the critical line.

The general solution of the differential equation is

$$
\Sigma(x)=c_{1} x^{-(1-\gamma) / 2}+c_{2} x^{-(1+\gamma) / 2},
$$

where $c_{1}$ and $c_{2}$ are the constants, and $\gamma=\sqrt{1-4 \lambda}$. The boundary conditions give

$$
c_{1} \frac{1-\gamma}{2} \mu^{1+\gamma}+c_{2} \frac{1+\gamma}{2} \mu^{1-\gamma}=0
$$




$$
c_{1}\left(1-\left(1+\frac{g_{4 F}}{\lambda}\right) \frac{1-\gamma}{2}\right) M^{-1+\gamma}+c_{2}\left(1-\left(1+\frac{g_{4 F}}{\lambda}\right) \frac{1+\gamma}{2}\right) M^{-1-\gamma}=0 .
$$

By combining these conditions, we obtain

$$
c_{2}\left[1-\frac{1-\gamma}{1+\gamma} \cdot \frac{1-\left(1+\frac{g_{4 F}}{\lambda}\right) \frac{1+\gamma}{2}}{1-\left(1+\frac{g_{4 F}}{\lambda}\right) \frac{1-\gamma}{2}}\left(\frac{\mu}{M}\right)^{2 \gamma}\right]=0 .
$$

The non-trivial solution exists if the inside of the square bracket vanishes. In the naive limit $\mu / M \rightarrow 0$, the condition $\gamma=0$, namely $\lambda=\lambda_{c}=1 / 4$, should be satisfied for any $g_{4 f}$ and $\lambda<1 / 4$. But, we have another solution for $g_{4 F} \geq 1 / 4$. The condition for non-trivial solution can be written as

$$
\frac{1-\gamma^{2}}{1+\gamma^{2}} \cdot \frac{1+\gamma-\frac{1}{2}\left(1-\gamma^{2}+4 g_{4 F}\right)}{1-\gamma-\frac{1}{2}\left(1-\gamma^{2}+4 g_{4 F}\right)}=\left(\frac{\mu}{M}\right)^{2 \gamma} .
$$

Taking the limit $\mu / M \rightarrow 0$ gives the another condition

$$
\lambda=\frac{1}{4}\left\{1-\left(\sqrt{4 g_{4 F}}-1\right)^{2}\right\} .
$$

We have obtained the critical line which is displayed in fig.(7.1).

The value of the critical gauge coupling $\lambda_{c}^{\prime}$ depends on the value of the effective four fermion coupling as

$$
\begin{cases}\lambda_{c}^{\prime}=\frac{1}{4}\left\{1-\left(\sqrt{4 g_{4 F}}-1\right)^{2}\right\} & g_{4 F}>\frac{1}{4} \\ \lambda_{c}^{\prime}=\frac{1}{4} & g_{4 F} \leq \frac{1}{4} .\end{cases}
$$

When the four fermion coupling is larger than $1 / 4$, the critical gauge coupling is reduced. The condensation scale of this channel is defined by using the phase diagram. We replace the gauge coupling constant by the running one given by the one-loop perturbative calculation. We, however, ignore the running of the massive gauge boson-fermion coupling, because the coupling does not become large. The reason is that the negative contribution to the $\beta$-function of the coupling of the broken gauge interaction can be neglected, since the gauge boson loop in the self-energy of the massive gauge bosons should contain at least one massive gauge boson. The condensation scale can be defined as the scale at which the gauge coupling takes critical value. The larger effective four fermion coupling constant means the smaller condensation scale and the larger anomalous dimension as $\gamma_{m}=1+\sqrt{1-4 \lambda_{c}^{\prime}}=\sqrt{4 g_{4 F}}$ for $g_{4 F} \geq 1 / 4$ [49]. We have a criterion for the attractive effective four fermion interaction to be significant to the dynamics: the coupling must be larger than $1 / 4$.

Now we evaluate the massive gauge boson effect on the dynamics of the channel with attractive gauge interaction and answer the question whether the large anomalous dimension dynamics due to the strong four fermion interaction can be realized in the channel. We can get a relation of the effective couplings at the scale $M$

$$
g_{4 F}^{\left(r_{c 1}^{H}\right)}=\lambda\left(r_{1}^{G}, r_{2}^{G}, r_{c}^{G}\right)-\lambda\left(r_{11}^{H}, r_{21}^{H}, r_{c 1}^{H}\right)
$$


from eqs. (7.11), (7.13) and (7.14), and now

$$
\lambda\left(r_{11}^{H}, r_{21}^{H}, r_{c 1}^{H}\right) \geq 0
$$

since the gauge interaction is considered to be attractive. Furthermore, the effective coupling $\lambda\left(r_{1}^{G}, r_{2}^{G}, r_{c}^{G}\right)$ at the scale $M$ is less than the critical coupling $\lambda_{c}=1 / 4$ because of the MAC hypothesis. Therefore, the four fermion coupling $g_{4 F}^{\left(r_{c 1}^{H}\right)}$ must be less than $1 / 4$ and we conclude that the attractive force by the massive gauge boson exchange is too small to significantly affect the dynamics in the channel with attractive gauge interaction. This means that the realistic technicolor dynamics with the large anomalous dimension due to the strong four fermion interaction cannot be realized in the low energy effective theory of tumbling gauge theories, provided that the mixing channels are ignored. We can also conclude that the MAC is not change, even if we consider the massive gauge boson effect.

This conclusion is also true when we consider a direct product gauge group. We consider that the gauge group $G \equiv G_{1} \otimes G_{2} \otimes \ldots \otimes G_{n}$ is broken to the gauge group $H \equiv H_{1} \otimes H_{2} \otimes \ldots \otimes H_{m}$ at the scale $M$. For example, we consider a channel

$$
R^{G}\{i\} \times R^{G}\{j\} \rightarrow R_{c}^{G}\{k\},
$$

where

$$
R^{G}\{i\} \equiv\left(r_{i_{1}}^{G_{1}}, r_{i_{2}}^{G_{2}}, \ldots, r_{i_{n}}^{G_{n}}\right)
$$

denote a representation. The representations of the group $G$ can be decomposed into the representations of the group $H$ as

$$
\begin{aligned}
R^{G}\{i\} & =\bigoplus_{\{p\}} R^{H}\{p\}, \\
R^{G}\{j\} & =\bigoplus_{\{q\}} R^{H}\{q\}, \\
R_{c}^{G}\{k\} & =\bigoplus_{\{l\}} R_{c}^{H}\{l\},
\end{aligned}
$$

where the summations are taken over the appropriate sets of the irreducible representations of $H,\{p\},\{q\}$ and $\{l\}$. If the channel mixing are ignored again, the decomposed representations of the mass functions in the effective action have the one-to-one correspondence to the channels in terms of the representations of $H$. We also obtain similar relations to determine the effective four fermion couplings. For example, for the channel

$$
R^{H}\{p\} \times R^{H}\{q\} \rightarrow R_{c}^{H}\{l\},
$$

we get the relation

$$
g_{4 F}^{\left(R^{H}\{l\}\right)}=\lambda\left(R^{G}\{i\}, R^{G}\{j\}, R_{c}^{G}\{k\}\right)-\lambda\left(R^{H}\{p\}, R^{H}\{q\}, R_{c}^{H}\{l\}\right),
$$

where the effective couplings are defined by

$$
\begin{aligned}
& \lambda\left(R^{G}\{i\}, R^{G}\{j\}, R_{c}^{G}\{k\}\right)=\sum_{\alpha=1}^{n} \lambda\left(r_{i_{\alpha}}^{G_{\alpha}}, r_{j_{\alpha}}^{G_{\alpha}}, r_{c k_{\alpha}}^{G_{\alpha}}\right), \\
& \lambda\left(R^{H}\{p\}, R^{H}\{q\}, R_{c}^{H}\{l\}\right)=\sum_{\beta=1}^{m} \lambda\left(r_{p_{\beta}}^{H_{\beta}}, r_{q_{\beta}}^{H_{\beta}}, r_{c l_{\beta}}^{H_{\beta}}\right) .
\end{aligned}
$$


Figure 7.2: Gap equation for fermions with Majorana mass.

Therefore, the four fermion coupling must be less than $1 / 4$ in the channel with attractive gauge interaction and the conclusion does not change. The attractive force of the massive gauge boson is too small to significantly affect the dynamics of the channel with attractive gauge interaction.

\subsection{The massive fermion effect}

Several fermions get their masses in the process of tumbling. In the original tumbling scenario it is naively assumed that the massive fermions do not cause significant effect on the lower energy dynamics. In this section we will argue the decoupling of the fermions which get Majorana masses in the process of tumbling ?. $^{2}$.

We examine the linearized ladder gap equation for the channel with the massive fermions,

$$
\begin{aligned}
& \Sigma_{A}\left(-q^{2}\right)= \\
& \quad+\lambda \int^{M^{2}} d k^{2} \frac{k^{2}}{\left(m_{1}^{2}+k^{2}\right)\left(m_{2}^{2}+k^{2}\right)}\left\{\theta\left(k^{2}-q^{2}\right)+\frac{k^{2}}{q^{2}} \theta\left(q^{2}-k^{2}\right)\right\} \Sigma_{A}\left(-k^{2}\right) \\
& \quad-\lambda \int^{M^{2}} d k^{2} \frac{m_{1} m_{2}}{\left(m_{1}^{2}+k^{2}\right)\left(m_{2}^{2}+k^{2}\right)}\left\{\theta\left(k^{2}-q^{2}\right)+\frac{k^{2}}{q^{2}} \theta\left(q^{2}-k^{2}\right)\right\}\left(\Sigma_{A}\left(-k^{2}\right)\right)^{*},
\end{aligned}
$$

where $M$ is the scale of the previous tumbling, $m_{1}$ and $m_{2}$ are the Majorana masses, $\lambda$ is the gauge coupling and $A$ is the index of the representation of condensate (fig.(7.2)). Here we neglect the massive gauge boson effect, because we have found in the previous section that such effect is small for $\lambda \geq 0$. This equation can be translated into a differential equation with boundary conditions,

$$
\begin{gathered}
\frac{d^{2}}{d x^{2}}(x \Sigma(x))+\lambda \frac{x \Sigma(x)-m_{1} m_{2} \Sigma(x)}{\left(m_{1}^{2}+x\right)\left(m_{2}^{2}+x\right)}=0, \\
\lim _{x \rightarrow 0} x^{2} \frac{d \Sigma}{d x}=0, \\
\lim _{x \rightarrow M^{2}} \frac{d}{d x}(x \Sigma(x))=0,
\end{gathered}
$$

where $x=q^{2}$ and index $A$ is omitted. We assume that the mass function is real.

First, we consider the case $m_{1}=m \simeq M$ and $m_{2}=0$. In this case the differential equation can be reduced to the Bessel differential equation

$$
\frac{d^{2} S}{d z^{2}}+\frac{1}{z} \frac{d S}{d z}+\left(1-\frac{1}{z^{2}}\right) S=0
$$

\footnotetext{
${ }^{3}$ Some fermions get Dirac masses (mixing Majorana masses) in the process of tumbling. These masses are due to the mixing of the condensation. We do not discuss the mixing, here.
} 
where $S(z)=z \Sigma(x)$ and $z=2 \sqrt{\lambda x / m^{2}}$. We can see that the condition

$$
J_{0}\left(2 \sqrt{\lambda M^{2} / m^{2}}\right)=0
$$

must be satisfied for the non-trivial solution to exist, where $J_{0}$ is the zero-th order Bessel function. The critical value of the coupling is defined by the lowest zero point of $J_{0}$ as

$$
\lambda_{c} \simeq 1.45 \frac{m^{2}}{M^{2}} \simeq 1.45
$$

and this is very large compared with the value of massless fermion case $1 / 4$.

Next, in the case $m_{1}=m_{2} \simeq M$, the equation can be reduced to the modified Bessel differential equation

$$
\frac{d^{2} S}{d z^{2}}+\frac{1}{z} \frac{d S}{d z}-\left(1+\frac{1}{z^{2}}\right) S=0
$$

We can see that there is no non-trivial solution which satisfies the boundary conditions.

Therefore, the channels containing the fermions with Majorana masses are unlikely to be the MAC. The fermions which get Majorana masses in the process of tumbling may decouple from the low energy dynamics. We have non-perturbative shown it. 


\section{Chapter 8}

\section{Conclusion}

The idea of the technicolor theory and the extended tehcnicolor theory is very beautiful. It can be expected that the theory solves some problems of the standard model. The hierarchical gauge symmetry breaking is expected in the extended technicolor theory to explain the hierarchy of the mass of the quarks and leptons. Tumbling gauge theory is a candidate of the dynamics of such hierarchical gauge symmetry breaking.

But some new problems peculiar to the technicolor theory is emerged. One of the problem is the flavor-changing neutral current problem. Extended technicolor theory results too large mixing of $K^{0}$ and $\bar{K}^{0}$ to be consistent with the experiment, for example. The problem is solved by considering the special technicolor dynamics with the large anomalous dimension. Therefore, it is expected that the large anomalous dimension is realized in the tumbling gauge theory.

We formulated the tumbling gauge theory using the Cornwall-Jackiw-Tomboulis effective action in the one gauge boson exchange approximation (ladder approximation). Non-perturbative effect of the massive particles which get their masses in the process of tumbling was estimated by using the formalism. We got the effective action at the low energy region of the tumbling gauge theory and obtained a formula to give the coupling constants of the effective four fermion interactions which is mediated by the massive gauge bosons. Upper bound on the coupling constants was obtained in the channels with attractive unbroken gauge interactions. The effective four fermion interactions are too weak to cause the significant effect on the dynamics. The large anomalous dimension due to the strong four fermion interaction with the attractive gauge interaction (gauged NambuJona-Lasinio type system) cannot be realized in the tumbling gauge theories. This result is important for the model building of the extended technicolor theory with tumbling dynamics. The possibility of walking dynamics (gauge theory with very slowly running coupling) in the tumbling gauge theory is not excluded.

We also estimated non-perturbative effect of the massive fermions (Majorana type) in the same framework. It was shown in one gauge boson exchange approximation that they decouple from the low energy dynamics \$. The channels containing massive fermions are hard to be MAC (most attractive channel). It can be expected that all the massive particles non-perturbatively decouple from the low energy dynamics. In the perturbative sense, the decoupling of the heavy particles from the low energy physics is well known as

\footnotetext{
${ }^{1}$ It must be noted that all the mixing effects were ignored in our argument.
} 
the decoupling theorem.

The recent phenomenological issues of the technicolor theory were also discussed. We discussed the relation between the technicolor theory and the recent precision experiments. The radiative correction due to the technicolor or extended technicolor dynamics is classified into two parts: oblique correction and non-oblique correction. The experi-

mental constraint on the oblique correction ( $S$ parameter) directory restricts the size of the technicolor sector. The number of the technicolor degrees of freedom and the number of the techni-flavor are restricted. The technicolor theory is disfavored by the experiments. Extended technicolor theory generally generates the large non-oblique correction. It was explained that the non-oblique correction on $Z b \bar{b}$ vertex due to the sideways and "diagonal" bosons are large, when the mass of the top quark is large. We showed that the correction on the vertex is too large to be consistent with the experiments. The extended technicolor theory is disfavored by the experiments.

All the results of this thesis are disadvantageous for the technicolor theory. If the nature selects the dynamical electroweak symmetry breaking and the dynamical generation of the ordinary fermion masses without the elementary scalar fields, there will be some new mechanisms. The present technique to analyze the non-perturbative dynamics of the gauge theory is poor. Therefore, the breakthrough in the technique can make possible to find the new mechanisms.

\section{Acknowledgments}

I am grateful to Professor Koichi Yamawaki for helpful encouragement and many discussions. I would like to thank Professor A.Ichiro Sanda, Yoshio Kikukawa, Masaharu Tanabashi, and Masayasu Harada for many discussions and suggestions. I also thank Sho Tsujimaru for careful reading of this thesis. I thank all the members of the elementary particle physics laboratory. 


\section{Bibliography}

[1] S.L.Glashow, Nucl. Phys. 22 (1961) 579; S.Weinberg, Phys. Rev. Lett. 19 (1967) 1264; A.Salam, Elementary Particle Theory, ed. N.Svarthold, Almquist and Wiksdls, Stochholm, 1968.

[2] P.W.Higgs, Phys. Lett. 12 (1964) 132, Phys. Rev. Lett. 13 (1964) 508; F.Englert and R.Brout, Phys. Rev. Lett. 13 (1964) 321, G.S.Guralnik, C.R.Hagen, and T.W.B.Kibble, Phys. Rev. Lett. 13 (1964) 585.

[3] M.Kobayashi and M.Maskawa, Prog. Theor. Phys. 49 (1973) 652.

[4] H.Georgi and S.L.Glashow, Phys. Rev. Lett. 32 (1974) 438. See P.Langacker, Phys. Rep. 72 (1981) 185 for review.

[5] S.Dimopoulos, L.J.Hall, and S.Raby, Phys. Rev. Lett. 68 (1992) 1984, Phys. Rev. D45 (1992) 4192, Phys. Rev. D46 (1992) R4793.

[6] S.Dimopoulos and L.Susskind, Nucl. Phys. B155 (1979) 237; E.Eichten and K.Lane, Phys. Lett. B90 (1980) 125.

[7] S.Weinberg, Phys. Rev. D19 (1979) 1277; L.Susskind, Phys. Rev. D20 (1979) 2619.

[8] V.A.Miransky, M.Tanabashi, and K.Yamawaki, Phys. Lett. 221B (1989) 177, Mod. Phys. Lett. A4 (1989) 1043; Y.Nambu, Enrico Fermi Inst. Preprint, EFI-89-08 (1989); J.Marciano, Phys. Rev. Lett. 62 (1989) 279; W.A.Bardeen, C.T.Hill, and M.Lindner, Phys. Rev. D41 (1990) 1647.

[9] S.Raby, S.Dimopoulos, and L.Susskind, Nucl. Phys. B169 (1980) 373.

[10] B.Holdom, Phys. Rev. D24 (1981) 1441; H.Georgi and S.L.Glashow, Phys. Rev. Lett. 47 (1981) 1511; K.Yamawaki and T.Yokota, Nucl. Phys. B223 (1983) 144.

[11] B.Holdom, Phys. Lett. 150B (1985) 301; K.Yamawaki, M.Bando, and K.Matumoto, Phys. Rev. Lett. 56 (1986) 1335; T.Akiba and T.Yanagida, Phys. Rett. 169B (1986) 432; T.Appelquist and L.C.R.Wijiewardhana, Phys. Rev. D35 (1987) 774.

[12] K.Yamawai, M.Bando, and K.Matumoto in [11]; V.A.Miransky and K.Yamawaki, Mod. Phys. Lett. A4 (1989) 129; K.Matumoto, Prog. Theor. Phys. 81 (1989) 277; T.Appelquist, T.Takeuchi, M.Einhorn, and L.C.R.Wijewardhana, Phys. Lett. B220 (1989) 223. 
[13] See for review, J.Gasser and H.Leutwyler, Phys. Rep. 87 (1982) 77.

[14] F.J.Dyson, Phys. Rev. 75 (1949) 1736; J.Schwinger, Proc. Nat. Acad. Sc. 37 (1951) 452.

[15] K.G.Wilson, Phys. Rev. 179 (1969) 1499; S.Weinberg, Phys. Rev. D8 (1973) 3497.

[16] H.Pagels and S.Stokar, Phys. Rev. D20 (1979) 2947.

[17] J.M.Cornwall R.Jackiw, and E.Tomboulis, Phys. Rev. D10 (1974) 2428;

C.D.Dominicis and P.C.Martin, Jour. of Math. Phys. 5 (1964) 31.

[18] D.Atkinson, J. Math. Phys. 28 (1987) 2494.

[19] T.Maskawa and H.Nakajima, Prog. Theor. Phys. 52 (1974) 1326; R.Fukuda and T.Kugo, Nucl. Phys. B117 (1976) 250.

[20] V.A.Miransky, Sov. J. Nucl. Phys. 38 (1983) 280; K.Higashijima, Phys. Rev. D29 (1984) 1228.

[21] E.Gildner, Phys. Rev. D14 (1976) 1667, Phys. Lett. B92 (1980) 111; G.t'Hooft, Recent Development in Gauge Theories, eds. G.t'Hooft et.al., Prenum Press, N.Y., (1980); M.Veltman, Act. Phys. Pol. B12 (1981) 277.

[22] S.Dimopoulos, Nucl. Phys. B168 (1980) 69.

[23] A.Manohar and H.Georgi, Nucl. Phys. B234 (1984) 189.

[24] R.Dashen, Phys. Rev. 183 (1969) 1245.

[25] S.Dimopoulos, S.Raby, and G.L.Kane, Nucl. Phys. B182 (1981) 77.

[26] M.L.Goldberger and S.B.Treiman, Phys. Rev. 110 (1958) 1178.

[27] J.Ellis, M.K.Gaillard, D.V.Nanopoulos, and P.Sikivie, Nucl. Phys. B182 (1981) 529.

[28] See figure 4 in F.Bergsma et.al. (CHARM collaboration), Phys. Lett. B157 (1985) 458 , and refernces therein.

[29] F.Abe et.al., Phys. Rev. Lett. 63 (1989) 1447.

[30] I.Abt et.al. (H1 collaboration), Nucl. Phys. B396 (1993) 3.

[31] D.Decamp et.al. (ALEPH collaboration), Phys. Rep. 216 (1992) 253.

[32] S.L.Glashow, J.Iliopoulos, and L.Maiani, Phys. Rev. D2 (1970) 1285.

[33] J.Pati and A.Salam, Phys. Rev. D10 (1974) 275.

[34] S.Dimopoulos and J.Ellis, Nucl. Phys. B182 (1981) 505.

[35] N.G.Deshpande and R.J.Johnson, Phys. Rev. D27 (1983) 1193. 
[36] K-I.Kondo, M.Tanabashi, and K.Yamawaki, Prog. Theor. Phys. 89 (1993) 1249.

[37] M.E.Peskin and T.Takeuchi, Phys. Rev. Lett. 65 (1990) 964, Phys. Rev. D46 (1992) 381.

[38] P.Sikivie, L.Susskind, M.Voloshin, and V.Zakharov, Nucl. Phys. B173 (1980) 189.

[39] T.Appelquist and G.Triantaphyllou, Phys. Lett. B278 (1992) 345; R.Sundrum and S.D.H.Hsu, Nucl. Phys. B391 (1993) 127.

[40] M.E.Peskin and T.Takeuchi, in Ref.[37]; P.Langacker, Conference summerly presented at 27th Rencontres de Moriond on Electroweak Interaction and Unified Theories, Published in Moriond 1992, Electroweak, 157; P.Privitera, Phys. Lett. B288 (1992) 227.

[41] E.Gates and J.Terning, Phys. Rev. Lett. 67 (1991) 1840.

[42] R.S.Chivukula, B.Selipsky, and E.H.Simmons, Phys. Rev. Lett. 69 (1992) 575; N.Kitazawa, Phys. Lett. B313 (1993) 395.

[43] B. Holdom, Phys. Lett. B259 (1991) 329.

[44] H.Georgi and S.L.Glashow, Phys. Rev. Lett. 32 (1974) 438.

[45] S.A.Frolov and A.A.Slavnov, Phys. Lett. B309 (1993) 344.

[46] G.Süalp and S.Kaptanoglu, Ann. Phys. 147 (1983) 460.

[47] T.Appelquist and J.Carazzone, Phys. Rev. D11 (1975) 2856.

[48] K.-I.Kondo, H.Mino, and K.Yamawaki, Phys. Rev. D39 (1989) 2430; T.Appelquist, M.Soldate, T.Takeuchi, and L.C.R. Wijewardhana, in Proc. Johns Hopkins Workshop on Current Problems in Particle Theory 12, Baltimore, June 8-10, 1988.

[49] V.Miransky and K.Yamawaki in [12]. 